\title{
ADMISSIBLE LEVEL osp(1|2) MINIMAL MODELS AND THEIR RELAXED HIGHEST WEIGHT MODULES
}

\author{
SIMON WOOD
}

\author{
School of Mathematics \\ Cardiff University \\ Cardiff \\ United Kingdom, CF24 4AG \\ woodsi@cardiff.ac.uk
}

\begin{abstract}
The minimal model osp $(1 \mid 2)$ vertex operator superalgebras are the simple quotients of affine vertex operator superalgebras constructed from the affine Lie super algebra $\widehat{\mathfrak{o s p}}(1 \mid 2)$ at certain rational values of the level $k$. We classify all isomorphism classes of $\mathbb{Z}_{2}$-graded simple relaxed highest weight modules over the minimal model $\mathfrak{o s p}(1 \mid 2)$ vertex operator superalgebras in both the Neveu-Schwarz and Ramond sectors. To this end, we combine free field realisations, screening operators and the theory of symmetric functions in the Jack basis to compute explicit presentations for the Zhu algebras in both the Neveu-Schwarz and Ramond sectors. Two different free field realisations are used depending on the level. For $k<-1$, the free field realisation resembles the Wakimoto free field realisation of affine $\mathfrak{s l}(2)$ and is originally due to Bershadsky and Ooguri. It involves 1 free boson (or rank 1 Heisenberg vertex algebra), one $\beta \gamma$ bosonic ghost system and one $b c$ fermionic ghost system. For $k>-1$, the argument presented here requires the bosonisation of the $\beta \gamma$ system by embedding it into an indefinite rank 2 lattice vertex algebra.
\end{abstract}

\section{Introduction}

The orthosymplectic Lie superalgebra $\mathfrak{o s p}(1 \mid 2)$ is the Lie superalgebra of endomorphisms of the vector superspace $\mathbb{C}^{1 \mid 2}$ that preserves the standard supersymmetric bilinear form on $\mathbb{C}^{1 \mid 2}$. It is arguably the easiest example of a finite-dimensional simple complex Lie superalgebra in Kac's classification [1]. The purpose of this article is to classify the simple relaxed highest weight modules over the minimal model $\mathfrak{o s p}(1 \mid 2)$ vertex operator superalgebras, that is, the simple quotient vertex operator superalgebras constructed from the affinisation of $\mathfrak{o s p}(1 \mid 2)$ at certain rational levels, called admissible levels.

Let $u, v$ be integers satisfying $u \geq 2, v \geq 1, u-v \in 2 \mathbb{Z}$ and $\operatorname{gcd}(u,(u-v) / 2)=1$

DOI: $10.1007 /$ s00031-020-09567-3

Received June 12, 2019. Accepted April 21, 2019.

Published online May 4, 2020.

Corresponding Author: Simon Wood, e-mail: woodsi@cardiff.ac.uk 
and let

$$
\begin{gathered}
k_{u, v}=\frac{u-3 v}{2 v}, \quad \lambda_{i, j}=\frac{i-1}{2}-\frac{1+(-1)^{i+j}}{4}-\frac{u}{2 v} j \\
s_{i, j}=\frac{i}{2}-\frac{u}{2 v} j, \quad q_{i, j}=\frac{(u j-v i)^{2}-4 v^{2}}{8 v^{2}}
\end{gathered}
$$

where $i$ and $j$ are integers. We denote the simple relaxed highest weight $\widehat{\mathfrak{o s p}}(1 \mid 2)$ modules as follows (see Section 2 for the precise definitions):

- In the Neveu-Schwarz sector, $\mathbb{Z}_{2}$-graded simple relaxed highest weight $\widehat{\mathfrak{o s p}}(1 \mid 2)$ modules at level $k \in \mathbb{C}$ are characterised by the simple osp $(1 \mid 2)$ weight module formed by the vectors of least conformal weight. Let $\alpha$ denote the simple root of $\mathfrak{o s p}(1 \mid 2)$.

(1) $\mathcal{A}_{\lambda}, \lambda \in \mathbb{Z}_{\geq 0}$, denotes the simple module whose space of vectors of least conformal weight is a finite-dimensional highest (and lowest) weight $\mathfrak{o s p}(1 \mid 2)$ module of highest weight $\lambda \alpha$ with a highest weight vector of even parity.

(2) $\mathcal{B}_{\lambda}^{+}, \lambda \in \mathbb{C} \backslash \mathbb{Z}_{\geq 0}$, denotes the simple module whose space of vectors of least conformal weight is an infinite-dimensional highest weight $\mathfrak{o s p}(1 \mid 2)$ module of highest weight $\lambda \alpha$ with a highest weight vector of even parity.

(3) $\mathcal{B}_{\lambda}^{-}, \lambda \in \mathbb{C} \backslash \mathbb{Z}_{\leq 0}$, denotes the simple module whose space of vectors of least conformal weight is an infinite-dimensional lowest-weight $\mathfrak{o s p}(1 \mid 2)$ module of lowest-weight $\lambda \alpha$ with a lowest-weight vector of even parity.

(4) $\mathcal{C}_{[\lambda], s},[\lambda] \in \mathbb{C} / 2 \mathbb{Z}, s \in \mathbb{C}$ satisfying $s^{2} \neq\left(\mu+\frac{1}{2}\right)^{2}$ for all $\mu \in[\lambda] \cup[\lambda+1]$, denotes the simple module whose space of vectors of least conformal weight is a dense $\mathfrak{o s p}(1 \mid 2)$ module, which is characterised by its weight support and the action of the $\mathfrak{o s p}(1 \mid 2)$ super-Casimir operator. The weight support of even vectors is $[\lambda] \alpha$, while the weight support of odd vectors is $[\lambda+1] \alpha$. The super-Casimir acts as multiplication by $s$ on even vectors and $-s$ on odd vectors.

- In the Ramond sector, $\mathbb{Z}_{2}$-graded simple relaxed highest weight $\widehat{\mathfrak{o s p}}(1 \mid 2)$ modules at level $k \in \mathbb{C}$ are characterised by the simple $\mathfrak{s l}(2)$ weight module formed by the vectors of least conformal weight. Since the even subalgebra of $\mathfrak{o s p}(1 \mid 2)$ is isomorphic to $\mathfrak{s l}(2)$, the simple root of this subalgebra is $2 \alpha$ (equivalently, $\alpha$ is the fundamental weight).

(5) $\mathcal{F}_{\lambda}, \lambda \in \mathbb{Z}_{\geq 0}$, denotes the simple module whose space of vectors of least conformal weight is even and is the finite-dimensional highest (and lowest) weight $\mathfrak{s l}(2)$ module of highest weight $\lambda \alpha$.

(6) $\mathcal{D}_{\lambda}^{+}, \lambda \in \mathbb{C} \backslash \mathbb{Z}_{\geq 0}$, denotes the simple module whose space of vectors of least conformal weight is even and is an infinite-dimensional highest weight $\mathfrak{s l}(2)$ module of highest weight $\lambda \alpha$.

(7) $\mathcal{D}_{\lambda}^{-}, \lambda \in \mathbb{C} \backslash \mathbb{Z}_{\leq 0}$, denotes the simple module whose space of vectors of least conformal weight is even and is an infinite-dimensional lowest-weight $\mathfrak{s l}(2)$ module of lowest-weight $\lambda \alpha$.

(8) $\mathcal{E}_{[\lambda], q},[\lambda] \in \mathbb{C} / 2 \mathbb{Z}, q \in \mathbb{C}$ satisfying $q \neq \mu(\mu+2)$ for all $\mu \in[\lambda]$, denotes the simple module whose space of vectors of least conformal weight is even and is a dense $\mathfrak{s l}(2)$ module, which is characterised by its weight support and the action of the $\mathfrak{s l}(2)$ quadratic Casimir operator. Its weight support is $[\lambda] \alpha$ and the $\mathfrak{s l}(2)$ Casimir operator acts as multiplication by $q$. 
We denote the simple quotient of the universal vertex operator superalgebra constructed from $\mathfrak{o s p}(1 \mid 2)$ at level $k_{u, v}$ by $\mathrm{B}_{0 \mid 1}(u, v)$. The main result of this paper can then be stated as follows.

Theorem. Every $\mathbb{Z}_{2}$-graded simple relaxed highest weight module over the minimal model osp $(1 \mid 2)$ vertex operator superalgebra, $\mathrm{B}_{0 \mid 1}(u, v)$, at level $k_{u, v}$ is isomorphic to one of the following or their parity reversals.

In the Neveu-Schwarz sector:

(1) $\mathcal{A}_{\lambda_{i, 0}}$, where $1 \leq i \leq u-1$ and $i$ is odd.

(2) $\mathcal{B}_{\lambda_{i, j}}^{+}$, where $1 \leq i \leq u-1,1 \leq j \leq v-1$ and $i+j$ is odd.

(3) $\mathcal{B}_{-\lambda_{i, j}}^{-}$, where $1 \leq i \leq u-1,1 \leq j \leq v-1$ and $i+j$ is odd.

(4) $\mathcal{C}_{[\lambda], s_{i, j}}$, where $[\lambda] \in \mathbb{C} / 2 \mathbb{Z}, 1 \leq i \leq u-1,1 \leq j \leq v-1$ and $i+j$ is odd.

In the Ramond sector:

(5) $\mathcal{F}_{\lambda_{i, 0}}$, where $1 \leq i \leq u-1$ and $i$ is even.

(6) $\mathcal{D}_{\lambda_{i, j}}^{+}$, where $1 \leq i \leq u-1,1 \leq j \leq v-1$ and $i+j$ is even.

(7) $\mathcal{D}_{-\lambda_{i, j}}^{-}$, where $1 \leq i \leq u-1,1 \leq j \leq v-1$ and $i+j$ is even.

(8) $\mathcal{E}_{[\lambda], q_{i, j}}$, where $[\lambda] \in \mathbb{C} / 2 \mathbb{Z}, 1 \leq i \leq u-1,1 \leq j \leq v-1$ and $i+j$ is even.

For the special case of $u=2$ and $v=4$, the above classification was proved in [2]. The (non-rigorous) classification of simple highest weight $\mathrm{B}_{0 \mid 1}(u, v)$ modules, that is, those listed as items (1), (2), (5) and (6) in the theorem above, has already been established in the physics literature by Fan and Yu, and Ennes and Ramallo [3], [4].

In the mathematics literature, the simple modules over $\mathrm{B}_{0 \mid 1}(u, 1)$ in the NeveuSchwarz sector were classified by Kac and Wang in [5] and those in the Ramond sector were later classified by Creutzig, Frohlich and Kanade [6]. In this case all simple modules are of the type listed in part 1 of the above classification theorem. For general $u, v$, the Neveu-Schwarz sector admissible simple highest weight $\widehat{\mathfrak{o s p}}(1 \mid 2)$ modules (modules whose span of analytic continuations of characters admit a closed action of the modular group) were classified by Kac and Wakimoto [7] and this classification matches the modules of points 1 and 2 . However, as far as the author is aware, this is the first rigorous classification of simple relaxed highest weight $\mathbb{Z}_{2}$-graded $\mathrm{B}_{0 \mid 1}(u, v)$ modules for general $u, v$.

The module classification presented above could now be combined with a number of recent developments. For example, recent results on character formulae for relaxed highest weight modules [8] applied to the classification could be used to repeat the Verlinde formula calculations of [2] using the standard module formalism [9]-[11]. An alternative approach to computing fusion rules and Verlinde formulae, realising the $\mathfrak{o s p}(1 \mid 2)$ minimal models as an extension of the tensor product of the Virasoro and $\mathfrak{s l}(2)$ minimal modules has also recently been completed [12]. A further application could be the classification of simple Whittaker $\widehat{\mathfrak{o s p}}(1 \mid 2)$ modules in analogy to the $\mathfrak{s l}(2)$ classification [13].

The minimal model $\mathfrak{o s p}(1 \mid 2)$ vertex operator superalgebra $\mathrm{B}_{0 \mid 1}(u, v)$ is a simple quotient of the universal $\mathfrak{o s p}(1 \mid 2)$ vertex operator superalgebra by an ideal generated by a singular vector. A natural strategy for classifying simple modules over $\mathrm{B}_{0 \mid 1}(u, v)$ is, therefore, to identify modules over the universal vertex operator superalgebra on which the ideal acts trivially. This annihilating ideals approach 
to module classification was first applied by Feigin, Nakanishi and Ooguri [14] to certain Virasoro minimal models. In the context of $\mathfrak{o s p}(1 \mid 2)$ this strategy was first used by Kac and Wang [5], as an application of their generalisation of Zhu algebras [15] to vertex operator superalgebras, to classify simple Neveu-Schwarz modules when $v=1$. These kinds of calculations require explicit formulae for the singular vector generating the ideal. Unfortunately, for $v>1$, the only known general formulae are formal expressions involving non-integer powers of affine $\mathfrak{o s p}(1 \mid 2)$ generators $[3,16]$ resembling those of Malikov, Feigin and Fuks [17] for affine Lie algebra singular vectors. Converting these formulae into expressions with non-negative integer powers is prohibitively laborious, so instead of using these formulae, the proof of the above classification theorem presented in this paper exploits a deep connection between free field realisations and symmetric functions to derive expressions which are more tractable. The use of free field realisations to study submodule structures goes all the way back to Feigin and Fuchs' analysis of Fock modules over the Virasoro algebra [18] and later to Wakimoto's free field realisation of $\mathfrak{s l}(2)$; see [19]. The connections to symmetric functions rely on screening operators, which were originally conceived by Dotsenko and Fateev [20] and later formalised by Tsuchiya and Kanie [21]. Finding tractable singular vector formulae in terms of symmetric functions has a similarly long history starting with the Virasoro algebra [22], [23] with later generalisations to other algebras, such as affine $\mathfrak{s l}(2)$ (see [24], [25]), the $N=1$ super Virasoro algebra [26]-[28] and the $W_{N}$-algebras [29], [30]. The presence of fermions (or odd fields) in vertex operator superalgebras necessitates the consideration of skew-symmetric functions as well as symmetric functions. Fortunately, this skew symmetry can be compensated for, so that symmetric function methods can still be applied after considering certain ideals, first considered by Feigin, Jimbo, Miwa and Mukhin [31], spanned by Jack symmetric polynomials whose parameter is negative rational.

The methods presented in this article were developed for and applied to the classification of simple modules of the triplet algebras [32], the Virasoro minimal models [33], the affine $\mathfrak{s l}(2)$ minimal models [25] and the $N=1$ superconformal minimal models [34]. In particular, [33], [25], [34] form a series aimed at systematising the classification of modules over vertex operator superalgebras which are nontrivial simple quotients of universal vertex operator superalgebras. A convenient property of these methods is that they work in essentially the same way not only in both the Neveu-Schwarz and Ramond sectors of a given algebra, but also across all examples of vertex operator superalgebras considered. Since all the algebras considered so far are rank 1, it will be interesting to see if these methods can be generalised to higher ranks as was recently done for the singular vector formulae of the $W_{N}$-algebras [30].

This article is organised as follows. In Section 1, we give a brief overview of $\mathfrak{o s p}(1 \mid 2)$, its affinisation $\widehat{\mathfrak{o s p}}(1 \mid 2)$ and modules over both of these algebras. The section concludes with the construction of the universal $\mathfrak{o s p}(1 \mid 2)$ vertex operator superalgebra $\mathrm{V}_{k}$ at level $k$ and the minimal model vertex operator superalgebra $\mathrm{B}_{0 \mid 1}(u, v)$ as a simple quotient of $\mathrm{V}_{k}$ at certain rational levels, termed admissible. In Section 2, we state explicit presentations of the untwisted and twisted Zhu algebras of $\mathrm{B}_{0 \mid 1}(u, v)$, that is, the Zhu algebras in the Neveu-Schwarz and Ramond sectors. 
The proof of these presentations is postponed to Section 4. These presentations are then used to prove the main result of the article, Theorem 11, that is, the classification of $\mathbb{Z}_{2}$-graded simple relaxed highest weight modules over $\mathrm{B}_{0 \mid 1}(u, v)$ and the rationality of $\mathrm{B}_{0 \mid 1}(u, v)$ in category $\mathscr{O}$. In Section 3 , we define the free field algebras and screening operators necessary for the free field realisation of $\mathrm{V}_{k}$, and derive identities for correlation functions in preparation for proving the presentations of the untwisted and twisted Zhu algebras. In Section 4, the presentations of the Zhu algebras are proved by evaluating the action of the zero modes of singular vectors of $\mathrm{V}_{k_{u, v}}$ on candidate relaxed highest weight vectors. This action of the zero modes depends polynomially on free field weights and, with the methods used here, can only be evaluated indirectly by determining certain zeros in the free field weights and showing that the zeros found saturate certain bounds. This necessitates splitting the calculation into two cases depending on whether $k<-1$ or $k>-1$ in order to assure that these bounds are indeed saturated.

Acknowledgements. The author would like to thank the following people for interesting discussions: Andreas Aaserud on polynomial rings, Tomoyuki Arakawa on universal enveloping algebras, Pierre Mathieu and Jorgen Rasmussen on fractional powers of screening operators and bosonising $\beta \gamma$ systems, and John Snadden on super-Casimir operators. Additionally, the author would like to thank David Ridout for interesting discussions on too many topics to list, and for the careful reading of a previous version of this article and giving helpful feedback. The author's research is supported by the Australian Research Council Discovery Early Career Researcher Award DE140101825 and the Discovery Project DP160101520.

\section{The $\mathfrak{o s p}(1 \mid 2)$ vertex operator superalgebra}

In this section we settle notation regarding the Lie superalgebra osp $(1 \mid 2)$, its affinisation and its associated vertex operator superalgebra, and recall known results. Since this article studies Lie superalgebras and vertex operator superalgebras, all vector spaces will be assumed to be complex vector superspaces, that is, graded by $\mathbb{Z}_{2}$. For any vector superspace $V$, we denote the subspace of even vectors by $V_{\overline{0}}$, the subspace of odd vectors by $V_{\overline{1}}$ and the parity reversal of $V$ by $\Pi V$. When considering vector spaces without any obvious superspace structure, then the grading will be assumed to be trivial unless stated otherwise, that is, the entire vector space will be assumed to be even (examples include $\widehat{\mathfrak{s l}}(2)$ or the $\beta \gamma$ ghost vertex algebra considered below). We refer readers unfamiliar with Lie superalgebras to [35] for an exhaustive discussion of the subject and to [2, Sect. 2.1] for a summary of $\mathfrak{o s p}(1 \mid 2)$ beyond that given below.

\section{The finite-dimensional simple Lie superalgebra osp $(1 \mid 2)$}

The orthosymplectic Lie superalgebra $\mathfrak{o s p}(1 \mid 2)$ is the Lie superalgebra preserving the standard supersymmetric bilinear form of $\mathbb{C}^{1 \mid 2}$, where supersymmetric means that the bilinear form is symmetric on the even subspace and skew-symmetric on the odd subspace. This Lie superalgebra is 5-dimensional and we choose the following basis: $\{e, x, h, y, f\}$, where $\operatorname{span}\{e, h, f\}=\mathfrak{o s p}(1 \mid 2)_{\overline{0}}$ and $\operatorname{span}\{x, y\}=$ 
$\mathfrak{o} \mathfrak{s}(1 \mid 2)_{\overline{1}}$. The non-vanishing (anti-) commutation relations in this basis are

$$
\begin{aligned}
{[h, e] } & =2 e, \quad[e, f] & =h, \quad[h, f] & =-2 f \\
{[e, y] } & =-x, \quad[h, x] & =x, \quad[h, y] & =-y, \quad[f, x]=-y \\
\{x, x\} & =2 e, \quad\{x, y\} & =h, \quad\{y, y\} & =-2 f .
\end{aligned}
$$

In their standard normalisation, the non-vanishing pairings of the invariant bilinear form on $\mathfrak{o s p}(1 \mid 2)$ are

$$
\kappa(h, h)=2, \quad \kappa(e, f)=\kappa(f, e)=1, \quad \kappa(x, y)=-\kappa(y, x)=2 .
$$

The (anti-)commutation relations (1) imply that the even subspace of $\mathfrak{o s p}(1 \mid 2)$ is isomorphic to $\mathfrak{s l}(2)$ and that $h$ spans a choice of Cartan subalgebra for both $\mathfrak{o s p}(1 \mid 2)$ and $\mathfrak{s l}(2)$. We denote by $\alpha$ the simple root corresponding to the root vector $x$, whose length with respect to the norm induced from (2) is $\|\alpha\|^{2}=1 / 2$. Note that, for the copy of $\mathfrak{s l}(2)$ sitting inside $\mathfrak{o s p}(1 \mid 2)$, the simple root is $2 \alpha$ and thus $\alpha$ is the fundamental weight of $\mathfrak{s l}(2)$.

Definition 1. A weight module over a Lie superalgebra is a module over that algebra which decomposes into a direct sum of simultaneous eigenspaces of the Cartan subalgebra. The weight support of a weight module is the set of all weights for which the corresponding weight space is non-trivial.

In this article we shall focus exclusively on weight modules and hence the subalgebras of the universal enveloping algebras of $\mathfrak{o s p}(1 \mid 2)$ and $\mathfrak{s l}(2)$ which preserve weight spaces will prove vital. These subalgebras are just the centralisers of the Cartan subalgebra.

Lemma 1. Let $\mathfrak{g}$ be a Lie superalgebra with choice of Cartan subalgebra $\mathfrak{h}$ and let

$$
\mathrm{C}(\mathfrak{h}, \mathfrak{g})=\{w \in \mathbf{U}(g):[h, w]=0, \forall h \in \mathfrak{h}\}
$$

be the centraliser of $\mathfrak{h}$ in the universal enveloping algebra of $\mathfrak{g}$.

(1) As an associative algebra

$$
\mathrm{C}(\mathfrak{h}, \mathfrak{o s p}(1 \mid 2)) \cong \mathbb{C}[h, \Sigma],
$$

where $\Sigma=x y-y x+1 / 2$ is the super-Casimir of $\mathfrak{o s p}(1 \mid 2)$.

(2) As an associative algebra

$$
\mathrm{C}(\mathfrak{h}, \mathfrak{s l}(2)) \cong \mathbb{C}[h, Q],
$$

where $Q=h^{2} / 2+e f+f e$ is the quadratic Casimir of $\mathfrak{s l}(2)$.

Part 2 of the above lemma is well known and part 1 is an immediate consequence of Pinczon's work [36] on the universal enveloping algebra of $\mathfrak{o s p}(1 \mid 2)$ and Leśniewski's discovery [37] of the super-Casimir of $\mathfrak{o s p}(1 \mid 2)$. While part 1 is surely also known, the author was not able to find a source and so a proof has been given for completeness. 
Proof. It was shown in [36] that the centre of the universal enveloping algebra is isomorphic to $\mathbb{C}\left[Q^{\mathfrak{o s p}(1 \mid 2)}\right]$, where

$$
Q^{\mathfrak{o s p}(1 \mid 2)}=\frac{1}{2} h^{2}+e f+f e-\frac{1}{2} x y+\frac{1}{2} y x=\frac{1}{2} \Sigma^{2}-\frac{1}{8}
$$

is the quadratic Casimir of $\mathfrak{o s p}(1 \mid 2)$. Further, it was shown in [37] that the superCasimir $\Sigma$ commutes with all even elements of $\mathfrak{o s p}(1 \mid 2)$ and anticommutes with all odd ones.

To prove the proposition, fix any Poincaré-Birkhoff-Witt ordering of the bases of $\mathfrak{o s p}(1 \mid 2)$ and $\mathfrak{s l}(2)$ and note that since $x^{2}=e$ and $y^{2}=-f$, the exponents of $x$ and $y$ in an element of the Poincaré-Birkhoff-Witt basis of $\mathfrak{o s p}(1 \mid 2)$ are at most 1. Every element of the Poincaré-Birkhoff-Witt basis is an eigenvector of the adjoint action of the Cartan subalgebra. Hence, the centraliser algebras $\mathrm{C}(\mathfrak{h}, \mathfrak{o s p}(1 \mid 2))$ and $\mathrm{C}(\mathfrak{h}, \mathfrak{s l}(2))$ are spanned by Poincaré-Birkhoff-Witt basis vectors whose eigenvalues are 0 . Within such basis vectors, every occurrence of the $f$ generator must be countered by an $e$ and vice versa for both $\mathfrak{o s p}(1 \mid 2)$ and $\mathfrak{s l}(2)$. Similarly, for $\mathfrak{o s p}(1 \mid 2)$, every occurrence of $y$ must be countered by an $x$ and vice versa. Using the commutation relations, the generators in these basis vectors can be reordered, so that that basis vectors become sums of monomials in $h, f e, y x$ for $\mathfrak{o} \mathfrak{s p}(1 \mid 2)$ and $h, f e$ for $\mathfrak{s l}(2)$. It then follows by direct computation that

$$
y x=\frac{1}{2}\left(h-\Sigma+\frac{1}{2}\right), \quad f e=-\frac{1}{4}\left(h+\Sigma+\frac{3}{2}\right)\left(h-\Sigma+\frac{1}{2}\right),
$$

for $\mathfrak{o s p}(1 \mid 2)$ and for $\mathfrak{s l}(2)$ that

$$
f e=\frac{1}{2}\left(Q-\frac{1}{2} h^{2}-h\right) .
$$

The algebraic independence of $h$ and $\Sigma$ then follow from the algebraic independence of $h$ and $Q$, since any algebraic relation of $h$ and $\Sigma$ would also imply a relation for $h$ and $Q$.

As mentioned in the proof above, the quadratic Casimir operator of $\mathfrak{o s p}(1 \mid 2)$ has the nice property of freely generating the centre of the universal enveloping algebra of $\mathfrak{o s p}(1 \mid 2)$, however, the super-Casimir operator $\Sigma$ shall prove to be far more convenient in the considerations that follow. For example, isomorphism classes of simple $\mathfrak{o s p}(1 \mid 2)$ weight modules can be characterised in terms of their weight support and the action of the super-Casimir operator $\Sigma$. Similarly, isomorphism classes of simple $\mathfrak{s l}(2)$ weight modules can be characterised in terms of their weight support and the action of the Casimir operator $Q$.

Theorem 2 (Block [38], [39]). Any simple $\mathfrak{s l}(2)$ weight module is isomorphic to one of the following:

(1) The simple $(\lambda+1)$-dimensional module $\overline{\mathcal{F}}_{\lambda}$ which is both highest and lowestweight, where $\lambda \in \mathbb{Z}_{\geq 0}$. The weights of the highest and lowest-weight vectors are $\lambda \alpha$ and $-\lambda \alpha$, respectively, and the eigenvalue of $Q$ is $\lambda(\lambda+2) / 2$. 
(2) The simple infinite-dimensional highest weight module $\overline{\mathcal{D}}_{\lambda}^{+}$, where $\lambda \in \mathbb{C} \backslash$ $\mathbb{Z}_{\geq 0}$. This module is generated by a highest weight vector $v_{\lambda}$ of weight $\lambda \alpha$, which therefore satisfies $e v_{\lambda}=0$ and $h v_{\lambda}=\lambda v_{\lambda}$. A basis is given by the weight vectors $\left\{f^{n} v_{\lambda}: n \in \mathbb{Z}_{\geq 0}\right\}$ and the eigenvalue of $Q$ is $\lambda(\lambda+2) / 2$. This is an example of a Verma module.

(3) The simple infinite-dimensional lowest-weight module $\overline{\mathcal{D}}_{\lambda}^{-}$, where $\lambda \in \mathbb{C} \backslash$ $\mathbb{Z}_{\leq 0}$. This module is generated by a lowest-weight vector $v_{\lambda}$ of weight $\lambda \alpha$, which therefore satisfies $f v_{\lambda}=0$ and $h v_{\lambda}=\lambda v_{\lambda}$. A basis is given by the weight vectors $\left\{e^{n} v_{\lambda}: n \in \mathbb{Z}_{\geq 0}\right\}$ and the eigenvalue of $Q$ is $\lambda(\lambda-2) / 2$. This is an example of a Verma module for which $f$ (as opposed to e) has been chosen as the simple root vector.

(4) The simple infinite-dimensional dense weight module $\overline{\mathcal{E}}_{\lambda, q}$, with $[\lambda] \in \mathbb{C} / 2 \mathbb{Z}$ and $q \in \mathbb{C}$ satisfying $q \neq \mu(\mu+2)$ for all $\mu \in[\lambda]$. This module is generated by a weight vector $v_{\lambda, q}$ that is neither highest nor lowest-weight, satisfying $h v_{\lambda, q}=\lambda v_{\lambda, q}$ and $Q v_{\lambda, q}=q v_{\lambda, q}$, and has a basis of weight vectors $\left\{v_{\lambda, q}\right.$, $\left.e^{n} v_{\lambda, q}, f^{n} v_{\lambda, q}: n \in \mathbb{Z}_{\geq 1}\right\}$.

Theorem 3. Any simple $\mathbb{Z}_{2}$-graded osp $(1 \mid 2)$ weight module is isomorphic to one of the following or its parity reversal:

(1) The simple $(2 \lambda+1)$-dimensional module $\overline{\mathcal{A}}_{\lambda}$ which is both highest and lowestweight, where $\lambda \in \mathbb{Z}_{\geq 0}$. The highest and lowest-weight vectors both have even parity and their respective weights are $\lambda \alpha$ and $-\lambda \alpha$. The eigenvalue of $\Sigma$ is $\lambda(\lambda+1) / 2$ on even vectors and $-\lambda(\lambda+1) / 2$ on odd vectors.

(2) The simple infinite-dimensional highest weight module $\overline{\mathcal{B}}_{\lambda}^{+}$, where $\lambda \in \mathbb{C} \backslash$ $\mathbb{Z}_{\geq 0}$. This module is generated by an even parity highest weight vector $v_{\lambda}$ of weight $\lambda \alpha$, which therefore satisfies $x v_{\lambda}=0$ and $h v_{\lambda}=\lambda v_{\lambda}$. A basis of weight vectors of this module is given by $\left\{y^{n} v_{\lambda}: n \in \mathbb{Z}_{\geq 0}\right\}$. The eigenvalue of $\Sigma$ is $\lambda(\lambda+1) / 2$ on even vectors and $-\lambda(\lambda+1) / 2$ on odd vectors. This is an example of a Verma module.

(3) The simple infinite-dimensional lowest-weight module $\overline{\mathcal{B}}_{\lambda}^{-}$, where $\lambda \in \mathbb{C} \backslash$ $\mathbb{Z}_{<0}$. This module is generated by an even parity lowest-weight vector $v_{\lambda}$, which therefore satisfies $y v_{\lambda}=0$ and $h v_{\lambda}=\lambda v_{\lambda}$. A basis of this module is given by the weight vectors $\left\{x^{n} v_{\lambda}: n \in \mathbb{Z}_{\geq 0}\right\}$. The eigenvalue of $\Sigma$ is $\lambda(\lambda-1) / 2$ on even vectors and $-\lambda(\lambda-1) / 2$ on odd vectors. This is an example of a Verma module for which $y$ has been chosen as the simple root vector.

(4) The simple infinite-dimensional dense weight module $\overline{\mathcal{C}}_{[\lambda], s}$, with $[\lambda] \in \mathbb{C} / 2 \mathbb{Z}$ and $s \in \mathbb{C}$ satisfying $s^{2} \neq(\mu+1 / 2)^{2}$ for any $\mu \in[\lambda] \cup[\lambda+1]$. This module is generated by an even parity weight vector $v_{\lambda, s}$ that is neither highest nor lowest-weight, satisfying $h v_{\lambda, s}=\lambda v_{\lambda, s}$ and $\Sigma v_{\lambda, s}=s v_{\lambda, s}$, and has a basis of weight vectors $\left\{v_{\lambda, s}, x^{n} v_{\lambda, s}, y^{n} v_{\lambda, s}: n \in \mathbb{Z}_{\geq 1}\right\}$.

Note that the parity reversal of $\overline{\mathcal{C}}_{[\lambda], s}$ is $\Pi \overline{\mathcal{C}}_{[\lambda], s} \cong \overline{\mathcal{C}}_{[\lambda+1],-s}$.

See [40] for a proof of Theorem 2 and a comprehensive discussion of $\mathfrak{s l}(2)$ module theory. The proof of the classification of simple $\mathfrak{o s p}(1 \mid 2)$ weight modules is similar to that of $\mathfrak{s l}(2)$ and was given in [2]. 


\section{The affinisation of $\mathfrak{o s p}(1 \mid 2)$}

The Lie superalgebra $\mathfrak{o s p}(1 \mid 2)$ can be affinised by constructing its loop algebra and centrally extending it. It is common to add a degree operator to ensure that the induced invariant bilinear form on the newly constructed affine Lie superalgebra is non-degenerate. Here, however, we shall always identify the action of such a degree operator on modules with that of the negative of the Virasoro $L_{0}$ operator of the Sugawara construction. We therefore omit the degree operator from the affinisation of $\mathfrak{o s p}(1 \mid 2)$. We consider two affinisations $\widehat{\mathfrak{o s p}}(1 \mid 2)_{0}$ and $\widehat{\mathfrak{o s p}}(1 \mid 2)_{1 / 2}$, identifiable by spectral flow isomorphisms, which we shall respectively refer to as the Neveu-Schwarz and Ramond affinisation. We shall suppress superscripts and just write $\widehat{\mathfrak{o s p}}(1 \mid 2)$ whenever the distinction between the two affinisations is not important. Though these two affinisations are isomorphic, their conformal gradings differ and thus so do the module categories which one considers for each algebra.

As vector spaces the Neveu-Schwarz and Ramond affinisations of $\mathfrak{o s p}(1 \mid 2)$ are given by

$$
\begin{aligned}
\widehat{\mathfrak{o s p}}(1 \mid 2)_{0} & =\mathfrak{o s p}(1 \mid 2) \otimes \mathbb{C}\left[t, t^{-1}\right] \oplus \mathbb{C} K \\
\widehat{\mathfrak{o s p}}(1 \mid 2)_{1 / 2} & =\operatorname{span}\{e, h, f\} \otimes \mathbb{C}\left[t, t^{-1}\right] \oplus \operatorname{span}\{x, y\} \otimes \mathbb{C}\left[t, t^{-1}\right] t^{1 / 2} \oplus \mathbb{C} K
\end{aligned}
$$

The (anti-) commutation relations of these two algebras are constructed from those of $\mathfrak{o s p}(1 \mid 2)$ and are given by

$$
\left[a_{m}, b_{n}\right]=[a, b] \otimes t^{m+n}+m \kappa(a, b) \delta_{m+n, 0}, \quad a_{m}=a \otimes t^{m}, b_{n}=b \otimes t^{n},
$$

where $a, b \in \mathfrak{o s p}(1 \mid 2)$ are homogeneous vectors of definite parity, [, ] denotes either the commutator or the anti-commutator depending on the parities of $a$ and $b$ and $m, n$ lie in $\mathbb{Z}$ or $\mathbb{Z}+1 / 2$ depending on the parities of $a, b$ and the algebra considered. Finally, $K$ is even and central. Here and hereafter we shall also suppress the $t$ variables and write $a_{m}$ instead of $a \otimes t^{m}$.

The Neveu-Schwarz and Ramond affinisations of osp(1|2) admit triangular decompositions as well as relaxed triangular decompositions. For the Neveu-Schwarz affinisation we choose the decompositions

$$
\begin{aligned}
\widehat{\mathfrak{o s p}}(1 \mid 2)_{0} & =\widehat{\mathfrak{o s p}}(1 \mid 2)_{0}^{-} \oplus \widehat{\mathfrak{h}} \oplus \widehat{\mathfrak{o s p}}(1 \mid 2)_{0}^{+}, \\
\widehat{\mathfrak{o s p}}(1 \mid 2)_{0}^{-} & =\operatorname{span}\left\{e_{-n}, x_{-n}, h_{-n}, y_{1-n}, f_{1-n}: n \in \mathbb{Z}^{+}\right\}, \\
\widehat{\mathfrak{o s p}}(1 \mid 2)_{0}^{+} & =\operatorname{span}\left\{e_{n-1}, x_{n-1}, h_{n}, y_{n}, f_{n}: n \in \mathbb{Z}^{+}\right\}, \\
\widehat{\mathfrak{h}} & =\operatorname{span}\left\{h_{0}, K\right\} \\
\widehat{\mathfrak{o s p}}(1 \mid 2)_{0} & =\widehat{\mathfrak{o s p}}(1 \mid 2)_{0}^{<} \oplus \widehat{\mathfrak{o s p}}(1 \mid 2)_{0}^{0} \oplus \widehat{\mathfrak{o s p}}(1 \mid 2)_{0}^{>} \\
\widehat{\mathfrak{o s p}}(1 \mid 2)_{0}^{<} & =\operatorname{span}\left\{e_{-n}, x_{-n}, h_{-n}, y_{-n}, f_{-n}: n \in \mathbb{Z}^{+}\right\} \\
\widehat{\mathfrak{o s p}}(1 \mid 2)_{0}^{>} & =\operatorname{span}\left\{e_{n}, x_{n}, h_{n}, y_{n}, f_{n}: n \in \mathbb{Z}^{+}\right\}, \\
\widehat{\mathfrak{o s p}}(1 \mid 2)_{0}^{0} & =\mathfrak{o s p}(1 \mid 2) \oplus \mathbb{C} K,
\end{aligned}
$$

where the decomposition on the left is the usual triangular decomposition into negative roots, a Cartan subalgebra and positive roots, and the decomposition on 
the right is a relaxed triangular decomposition. For the Ramond affinisation we choose the decompositions

$$
\begin{aligned}
\widehat{\mathfrak{o s p}}(1 \mid 2)_{1 / 2} & =\widehat{\mathfrak{o s p}}(1 \mid 2)_{1 / 2}^{-} \oplus \widehat{\mathfrak{h}} \oplus \widehat{\mathfrak{o s p}}(1 \mid 2)_{1 / 2}^{+}, \\
\widehat{\mathfrak{o s p}}(1 \mid 2)_{1 / 2}^{-} & =\operatorname{span}\left\{e_{-n}, x_{1 / 2-n}, h_{-n}, y_{1 / 2-n}, f_{1-n}: n \in \mathbb{Z}^{+}\right\}, \\
\widehat{\mathfrak{o s p}}(1 \mid 2)_{1 / 2}^{+} & =\operatorname{span}\left\{e_{n-1}, x_{n-1 / 2}, h_{n}, y_{n-1 / 2}, f_{n}: n \in \mathbb{Z}^{+}\right\}, \\
\widehat{\mathfrak{h}} & =\operatorname{span}\left\{h_{0}, K\right\} \\
\widehat{\mathfrak{o s p}}(1 \mid 2)_{1 / 2} & =\widehat{\mathfrak{o s p}}(1 \mid 2)_{1 / 2}^{<} \oplus \widehat{\mathfrak{o s p}}(1 \mid 2)_{1 / 2}^{0} \oplus \widehat{\mathfrak{o s p}}(1 \mid 2)_{1 / 2}^{>}, \\
\widehat{\mathfrak{s s p}}(1 \mid 2)_{1 / 2}^{<} & =\operatorname{span}\left\{e_{-n}, x_{1 / 2-n}, h_{-n}, y_{1 / 2-n}, f_{-n}: n \in \mathbb{Z}^{+}\right\} \\
\widehat{\mathfrak{o s p}}(1 \mid 2)_{1 / 2}^{>} & =\operatorname{span}\left\{e_{n}, x_{n-1 / 2}, h_{n}, y_{n-1 / 2}, f_{n}: n \in \mathbb{Z}^{+}\right\} \\
\widehat{\mathfrak{o s p}}(1 \mid 2)_{1 / 2}^{0} & =\mathfrak{s l}(2) \oplus \mathbb{C} K,
\end{aligned}
$$

where the decomposition on the left is the usual triangular decomposition and the decomposition on the right is a relaxed triangular decomposition. Finally, the parabolic subalgebras are denoted by

$$
\widehat{\mathfrak{o s p}}(1 \mid 2)_{\epsilon}^{\geq}=\widehat{\mathfrak{o s p}}(1 \mid 2)_{\epsilon}^{0} \oplus \widehat{\mathfrak{o s p}}(1 \mid 2)_{\epsilon}^{>}
$$

Definition 2. Let $\mathcal{M}$ be an $\widehat{\mathfrak{o s p}}(1 \mid 2)_{\epsilon}$ module. A relaxed highest weight vector $m \in$ $\mathcal{M}$ is a simultaneous eigenvector of the Cartan subalgebra $\widehat{\mathfrak{h}}$ which is annihilated by $\widehat{\mathfrak{o s p}}(1 \mid 2)_{\epsilon}^{>}$. Further, $\mathcal{M}$ is said to be a relaxed highest weight module if it is generated by a relaxed highest weight vector and is said to be a relaxed Verma module if it is isomorphic to

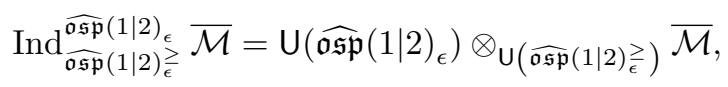

where $\mathrm{U}\left(\widehat{\mathfrak{o s p}}(1 \mid 2)_{\epsilon}\right)$ denotes the universal enveloping algebra and $\overline{\mathcal{M}}$ is some simple $\widehat{\mathfrak{o s p}}(1 \mid 2)_{\epsilon}^{0}$ weight module upon which $\widehat{\mathfrak{o s p}}(1 \mid 2)_{\epsilon}^{>}$acts trivially.

Since in the Neveu-Schwarz and Ramond sectors, respectively, we have

$$
\begin{aligned}
\widehat{\mathfrak{o s p}}(1 \mid 2)_{0}^{0} \cong \mathfrak{o s p}(1 \mid 2) \oplus \mathbb{C} K, \\
\widehat{\mathfrak{o s p}}(1 \mid 2)_{1 / 2}^{0} \cong \mathfrak{s l}(2) \oplus \mathbb{C} K,
\end{aligned}
$$

Neveu-Schwarz and Ramond Verma modules are respectively induced from $\mathfrak{o} \mathfrak{s p}(1 \mid 2)$ and $\mathfrak{s l}(2)$ modules on which the central element $K$ to act as $k \cdot \mathrm{id}, k \in \mathbb{C}$. For Ramond Verma modules, the $\mathfrak{s l}(2)$ modules they are induced from will be assigned even parity.

The above triangular decompositions suggest certain natural module categories within which to consider weight modules.

Definition 3. Category $\mathscr{R}$ is the category of $\widehat{\mathfrak{o s p}}(1 \mid 2)_{\epsilon}$ modules $\mathcal{M}$ which satisfy the following axioms: 
- $\mathcal{M}$ is $\mathbb{Z}_{2}$-graded.

- $\mathcal{M}$ is finitely generated.

- $\mathcal{M}$ is a weight module (the action of the Cartan subalgebra is semisimple).

- The action of $\widehat{\mathfrak{o s p}}(1 \mid 2)_{\epsilon}^{>}$is locally nilpotent: For any $m \in \mathcal{M}$, the space $\mathrm{U}\left(\widehat{\mathfrak{o s p}}(1 \mid 2)_{\epsilon}^{>}\right) \cdot m$ is finite-dimensional.

The morphisms are the $\widehat{\mathfrak{o s p}}(1 \mid 2)_{\epsilon}$ module homomorphism between these modules. Category $\mathscr{O}$ is the full subcategory of category $\mathscr{R}$ whose modules satisfy the additional property

- The action of $\widehat{\mathfrak{o s p}}(1 \mid 2)_{\epsilon}^{+}$is locally nilpotent.

All relaxed highest weight modules belong to category $\mathscr{R}$ and highest weight modules belong to category $\mathscr{O}$ and thus also $\mathscr{R}$. Additionally, since $\widehat{\mathfrak{o s p}}(1 \mid 2)_{\epsilon}$ has finite-dimensional root spaces (if one grades by both $\mathfrak{o s p}(1 \mid 2)$ and conformal weight), finite generation implies that each module in $\mathscr{R}$ has finite-dimensional weight spaces (again, if one grades by both $\mathfrak{o s p}(1 \mid 2)$ and conformal weight). If one grades only by conformal weight or only by $\mathfrak{o s p}(1 \mid 2)$ weight, then the weight spaces will be infinite-dimensional in general. Finally, the axioms also imply that every module in category $\mathscr{R}$ contains a relaxed highest weight vector, thus the simple objects of category $\mathscr{R}$ are simple relaxed highest weight modules.

Definition 4. We denote the unique simple quotients by maximal proper submodules of the inductions of the modules of Theorems Theorems 2 and 3 by the same symbols with the overline removed. To simplify notation, we suppress the algebras appearing in the super- and subscript of $\operatorname{Ind}_{\widehat{\mathfrak{o s p}}(1 \mid 2)_{\epsilon}}^{\widehat{\mathfrak{o s p}}(1 \mid 2)_{\epsilon}}$.

- In the Neveu-Schwarz sector:

(1) $\mathcal{A}_{\lambda}$, where $\lambda \in \mathbb{Z}_{\geq 0}$, is the simple quotient of Ind $\overline{\mathcal{A}}_{\lambda}$.

(2) $\mathcal{B}_{\lambda}^{+}$, where $\lambda \in \mathbb{C} \backslash \mathbb{Z}_{\geq 0}$, is the simple quotient of Ind $\overline{\mathcal{B}}_{\lambda}^{+}$.

(3) $\mathcal{B}_{\lambda}^{-}$, where $\lambda \in \mathbb{C} \backslash \mathbb{Z}_{\leq 0}$, is the simple quotient of Ind $\overline{\mathcal{B}}_{\lambda}^{-}$.

(4) $\mathcal{C}_{[\lambda], s}$, with $[\lambda] \in \mathbb{C} / 2 \mathbb{Z}, s \in \mathbb{C}$ satisfying $s^{2} \neq(\mu+1 / 2)^{2}$ for any $\mu \in$ $[\lambda] \cup[\lambda+1]$, is the simple quotient of Ind $\overline{\mathcal{C}}_{[\lambda], s}$.

- In the Ramond sector:

(5) $\mathcal{F}_{\lambda}$, where $\lambda \in \mathbb{Z}_{\geq 0}$, is the simple quotient of Ind $\overline{\mathcal{F}}_{\lambda}$.

(6) $\mathcal{D}_{\lambda}^{+}$, where $\lambda \in \mathbb{C} \backslash \mathbb{Z}_{\geq 0}$, is the simple quotient of Ind $\overline{\mathcal{D}}_{\lambda}^{+}$.

(7) $\mathcal{D}_{\lambda}^{-}$, where $\lambda \in \mathbb{C} \backslash \mathbb{Z}_{\leq 0}$, is the simple quotient of Ind $\overline{\mathcal{D}}_{\lambda}^{-}$.

(8) $\mathcal{E}_{[\lambda], q}$, with $[\lambda] \in \mathbb{C} / 2 \mathbb{Z}, q \in \mathbb{C}$ satisfying $q \neq \mu(\mu+2)$ for any $\mu \in[\lambda]$, is the simple quotient of Ind $\overline{\mathcal{E}}_{[\lambda], q}$.

Finally, before considering the vertex operator superalgebras that can be constructed from $\widehat{\mathfrak{o s p}}(1 \mid 2)$, we introduce a family of isomorphisms $\sigma^{\ell}, \quad \ell \in \frac{1}{2} \mathbb{Z}$, called spectral flow. These relate Neveu-Schwarz and Ramond affinisations of $\mathfrak{o s p}(1 \mid 2)$. The images of the basis vectors of $\widehat{\mathfrak{o s p}}(1 \mid 2)$ under $\sigma^{\ell}$ are

$$
\begin{array}{lll}
\sigma^{\ell}\left(e_{n}\right)=e_{n-2 \ell}, & \sigma^{\ell}\left(x_{n}\right)=x_{n-\ell}, & \sigma^{\ell}\left(h_{n}\right)=h_{n}-2 \ell \delta_{n, 0} K, \\
\sigma^{\ell}(K)=K, & \sigma^{\ell}\left(y_{n}\right)=y_{n+\ell}, & \sigma^{\ell}\left(f_{n}\right)=f_{n+2 \ell} .
\end{array}
$$


Clearly, the spectral flow isomorphism $\sigma^{\ell}$ is an automorphism relating the Neveu-Schwarz and Ramond affinisations to themselves if $\ell \in \mathbb{Z}$, otherwise it maps from the Neveu-Schwarz to the Ramond affinisation and vice versa. Algebra isomorphisms such as the spectral flow isomorphism $\sigma^{\ell}$ lead one naturally to consider modules whose algebra action has been twisted by such an automorphism.

Definition 5. Let $A$ and $B$ be Lie superalgebras, $\phi: A \rightarrow B$ an isomorphism and let $M$ be a module over $B$, then we define, $\phi^{-1} M$, the twist of $M$ by $\phi$, to be the $A$-module which as a vector superspace is just $M$ but on which the action of $A$ is defined to be

$$
a \cdot{ }_{\phi} m=\phi(a) m, \quad \forall a \in A, \forall m \in M .
$$

Because the spectral flow isomorphisms do not preserve the triangular decompositions (3) or (4), the twist of a module in category $\mathscr{R}$ need not lie in category $\mathscr{R}$. However, the algebra isomorphism $\zeta$ defined by

$$
\begin{array}{lll}
\zeta\left(e_{n}\right)=-f_{n+1}, & \zeta\left(x_{n}\right)=-y_{n+1 / 2}, & \zeta\left(h_{n}\right)=-h_{n}+2 \delta_{n, 0} K, \\
\zeta(K)=K, & \zeta\left(y_{n}\right)=x_{n-1 / 2}, & \zeta\left(f_{n}\right)=-e_{n-1} .
\end{array}
$$

preserves the standard triangular decompositions of $\widehat{\mathfrak{o s p}}(1 \mid 2)$, that is, $\zeta\left(\widehat{\mathfrak{o s p}}(1 \mid 2)_{\epsilon}^{ \pm}\right)$ $=\widehat{\mathfrak{o s p}}(1 \mid 2)_{1 / 2-\epsilon}^{ \pm}$and $\zeta(\widehat{\mathfrak{h}})=\widehat{\mathfrak{h}}$. Twisting by $\zeta$ therefore defines functors mapping between the Neveu-Schwarz and Ramond sectors of category $\mathscr{O}$. In particular, the twist of a highest weight Neveu-Schwarz module at level $k$ of highest weight $\lambda \alpha$ is a highest weight Ramond module at level $k$ of highest weight $(k-\lambda) \alpha$.

Remark 1. Our choice of representation category is informed by physics considerations coming from conformal field theory as well as technical considerations coming from Zhu's algebra, an associative algebra to be discussed below. A necessary condition for the consistency of a conformal field theory is that the representation category be closed under fusion and conjugation. Additionally, one generally requires characters to be well defined and to behave well under modular transformations so that modular invariants can be identified and fusion rules at the level of the Grothendieck group can be computed from Verlinde-like formulae. Neither closure under fusion nor conjugation appear to be satisfied for category $\mathscr{O}$ when considering non-integral admissible levels, see [11] for the case of $\mathfrak{s l}(2)$, [41] for the $\beta \gamma$ ghosts or [2] for $\mathfrak{o s p}(1 \mid 2)$ at level $k=-5 / 4$. While the generalisation to category $\mathscr{R}$ yields closure under conjugation, the Verlinde-like formulae in [2], [11], [41], computed using the standard module formalism, indicate that category $\mathscr{R}$ does not close under fusion and indeed also that the action of the modular group does not close on the span of category $\mathscr{R}$ characters.

The reason for focusing on category $\mathscr{R}$ is that modules in category $\mathscr{R}$ will always contain relaxed highest weight vectors and Zhu's algebra can be interpreted as the associative algebra of zero modes of fields acting on relaxed highest weight vectors. As Zhu algebra methods are blind to modules not containing relaxed highest weight vectors, category $\mathscr{R}$ is the largest category in which Zhu algebras can be used for module classification.

The success of the standard module formalism as a conjectured generalisation of the Verlinde formula for rational theories suggests that the natural module 
category to work with is the smallest category containing $\mathscr{R}$, all twists of $\mathscr{R}$ by the spectral flow isomorphisms $\sigma^{\ell}$ and all extensions of modules in these categories.

\section{The affine $\mathfrak{o s p}(1 \mid 2)$ vertex operator superalgebra}

The affine $\mathfrak{o s p}(1 \mid 2)$ vertex operator superalgebras are constructed by inducing from the trivial osp $(1 \mid 2)$ module. Let $\mathbb{C}_{k}, k \in \mathbb{C} \backslash\{-3 / 2\}$ be the 1 -dimensional $\widehat{\mathfrak{o s p}}(1 \mid 2)_{0}^{0}$ module on which $\mathfrak{o s p}(1 \mid 2)$ acts trivially and on which the central element $K$ acts as $k \cdot$ id. As an $\widehat{\mathfrak{o s p}}(1 \mid 2)_{0}$ module, the universal $\mathfrak{o s p}(1 \mid 2)$ vertex operator superalgebra $\mathrm{V}_{k}$ is given by the relaxed Verma module induced from $\mathbb{C}_{k}$, that is,

$$
\mathrm{V}_{k}=\operatorname{Ind}_{\widehat{\mathfrak{o s p}}(1 \mid 2)_{0}^{\geq}}^{\widehat{\mathfrak{o s p}}(1 \mid 2)_{0}} \mathbb{C}_{k}
$$

As a vertex operator superalgebra $V_{k}$ is freely generated under normal ordering and taking derivatives by 5 fields, labelled by the basis vectors of $\mathfrak{o s p}(1 \mid 2)$, subject to the operator product relations

$$
a(z) b(w) \sim \frac{\kappa(a, b)}{(z-w)^{2}}+\frac{[a, b](w)}{z-w}, \quad a, b \in \mathfrak{o s p}(1 \mid 2) .
$$

The Virasoro field, whose Laurent expansion coefficients generate the Virasoro algebra, is given by the standard Sugawara construction.

$$
\begin{aligned}
T(z)=\frac{1}{2 k+3}\left(\frac{1}{2}: h(z)^{2}:+: e(z) f(z):\right. & +: f(z) e(z): \\
& \left.-\frac{1}{2}: x(z) y(z):+\frac{1}{2}: y(z) x(z):\right) .
\end{aligned}
$$

We follow the definition given by Frenkel and Ben-Zvi [42, Chap. 5.1], augmented to include a $\mathbb{Z}_{2}$ grading by parity, for modules over a vertex operator superalgebra. Note that this implies that a module over $V_{k}$ is just a smooth $\mathbb{Z}_{2^{-}}$ graded level $k \widehat{\mathfrak{o s p}}(1 \mid 2)$-module and vice versa.

Corollary 4. All modules in the Neveu-Schwarz sector of $\mathscr{R}$ are modules over $\mathrm{V}_{k}$, while all modules in the Ramond sector of $\mathscr{R}$ are modules twisted by the parity automorphism over $\mathrm{V}_{k}$, that is, modules for which the action of odd fields has half integer monodromy about 0 .

We call a non-zero vector of a $\mathrm{V}_{k}$ module singular if it is a simultaneous eigenvector of $h_{0}$ and $L_{0}$, and is annihilated by all positive root vectors, that is, by $\widehat{\mathfrak{o s p}}(1 \mid 2)_{0}^{+}$. The highest weight vectors which generate Verma modules are prominent examples of singular vectors.

Proposition 5 (Gorelik and Kac [43]). The vertex operator superalgebra $\mathrm{V}_{k}, k \in$ $\mathbb{C} \backslash\{-3 / 2\}$ has a non-trivial proper ideal if and only if there exist integers $u \geq 2$, $v \geq 1$ satisfying $u-v \in 2 \mathbb{Z}$ and $\operatorname{gcd}(u,(u-v) / 2)=1$ such that

$$
2 k+3=\frac{u}{v} .
$$


Further, the ideal is unique (the only other ideals are the trivial one and the vertex operator superalgebra itself) and it is generated by a singular vector $\chi_{u, v}$ of $\mathfrak{o s p}(1 \mid 2)$ weight $(u-1) \alpha$ and conformal weight $(u-1) v / 2$.

Note that since the ideal of Theorem 5 is unique, $\mathrm{V}_{k}$ admits no singular vectors other than $\chi_{u, v}$ and the vacuum vector, up to rescaling. We refer to the levels satisfying the conditions of Theorem 5 as admissible and parametrise them as

$$
k_{u, v}=\frac{u-3 v}{2 v} .
$$

Definition 6. Let $u \geq 2, v \geq 1$ be integers satisfying

$$
u-v \in 2 \mathbb{Z} \quad \text { and } \operatorname{gcd}(u,(u-v) / 2)=1
$$

The minimal model $\mathfrak{o s p}(1 \mid 2)$ vertex operator superalgebra $\mathrm{B}_{0 \mid 1}(u, v)$ is defined to be the unique simple quotient of the universal $\mathfrak{o s p}(1 \mid 2)$ vertex operator superalgebra $\mathrm{V}_{k_{u, v}}$ by its maximal proper ideal:

$$
\mathrm{B}_{0 \mid 1}(u, v)=\frac{\bigvee_{k_{u, v}}}{\left\langle\chi_{u, v}\right\rangle} .
$$

Our choice of notation for $\mathrm{B}_{0 \mid 1}(u, v)$ mimics Kac's notation for $\mathfrak{o s p}(1 \mid 2)$ in his classification of simple Lie superalgebras [1]. Here and hereafter it will always be assumed that the variables $u$ and $v$ satisfy the conditions of Theorem 5 and Definition 6 . In the context of any formulae that $u, v$ appear in, the level will always be taken to satisfy Theorem 5 . Since $\mathrm{B}_{0 \mid 1}(u, v)$ is a quotient of $\mathrm{V}_{k_{u, v}}$, a module over $\mathrm{V}_{k_{u, v}}$ is also a module over $\mathrm{B}_{0 \mid 1}(u, v)$ if and only if the ideal $\left\langle\chi_{u, v}\right\rangle$ acts trivially. This clearly holds in both the Neveu-Schwarz and the Ramond sectors, that is, for both untwisted and twisted modules.

\section{Zhu algebras and module classification}

Zhu algebras are associative algebras constructed from vertex operator superalgebras. They can be interpreted as the algebras of zero modes of vertex operator superalgebra fields acting on relaxed highest weight vectors. They are invaluable aides to module classification, because there is a one-to-one correspondence between simple Zhu algebra modules and simple relaxed highest weight vertex operator superalgebra modules. This is due to the fact that the space of relaxed highest weight vectors of a simple relaxed highest weight vertex operator superalgebra module is naturally a Zhu algebra module and conversely any Zhu algebra module can be induced to a vertex operator superalgebra module whose space of relaxed highest weights is the Zhu algebra module. As their name suggests, Zhu algebras were originally considered by Zhu [15] for vertex operator algebras. Zhu's work was then later generalised to untwisted modules over vertex operator superalgebras by Kac and Wang [5] and to twisted vertex operator superalgebra modules by Dong, $\mathrm{Li}$ and Mason [44]. We refer to [34, App. A] for the definitions and conventions used here. 
Proposition 6. For $k \in \mathbb{C} \backslash\{-3 / 2\}$, the untwisted and twisted Zhu algebras of $\mathrm{V}_{k}$ are

$$
\mathrm{Zhu}\left[\mathrm{V}_{k}\right] \cong \mathrm{U}(\mathfrak{o s p}(1 \mid 2)), \quad \mathrm{Zhu}^{\tau}\left[\mathrm{V}_{k}\right] \cong \mathrm{U}(\mathfrak{s l}(2))
$$

The untwisted case was proved in [5] and the twisted case in [2]; both follow from the same reasoning as in [45] where non-super affine Lie algebras were considered.

Proposition 7. Let $u \geq 2, v \geq 1$ be integers satisfying the condition (8). The untwisted and twisted Zhu algebras of $\mathrm{B}_{0 \mid 1}(u, v)$ are

$$
\begin{aligned}
\operatorname{Zhu}\left[\mathrm{B}_{0 \mid 1}(u, v)\right] \cong \frac{\mathrm{U}(\mathfrak{o s p}(1 \mid 2))}{\left\langle\left[\chi_{u, v}\right]\right\rangle}, \\
\operatorname{Zhu}^{\tau}\left[\mathrm{B}_{0 \mid 1}(u, v)\right] \cong \begin{cases}\frac{\mathrm{U}(\mathfrak{s l}(2))}{\left\langle\left[\chi_{u, v}\right]^{\tau}\right\rangle}, & u, v \text { odd }, \\
\frac{\mathrm{U}(\mathfrak{s l}(2))}{\left\langle\left[y_{0} \chi_{u, v}\right]^{\tau}\right\rangle}, & u, v \text { even },\end{cases}
\end{aligned}
$$

where $\left\langle\left[\chi_{u, v}\right]\right\rangle$ and $\left\langle\left[\chi_{u, v}\right]^{\tau}\right\rangle\left(\right.$ or $\left.\left\langle\left[y_{0} \chi_{u, v}\right]^{\tau}\right\rangle\right)$ are the two-sided ideals generated by the images of the singular vector $\chi_{u, v}\left(\right.$ or $\left.y_{0} \chi_{u, v}\right)$ in the algebra $\mathrm{Zhu}\left[\mathrm{B}_{0 \mid 1}(u, v)\right]$ and $\mathrm{Zhu}^{\tau}\left[\mathrm{B}_{0 \mid 1}(u, v)\right]$, respectively.

The above presentations for the untwisted and twisted Zhu algebras were proved in [2]. We will be computing the image of the singular vector in the Zhu algebras by evaluating certain polynomial constraints. These constraints are only meaningful once one knows that the image of the singular vector is non-zero.

Lemma 8. Let $u \geq 2, v \geq 1$ be integers satisfying the condition (8). The image of the singular vector $\chi_{u, v}$ of $\mathrm{V}_{k_{u, v}}$ in the untwisted and twisted Zhu algebras is non-zero.

Proof. We prove the lemma by contradiction. If the image of the singular vector were zero in the untwisted or twisted Zhu algebras, then the two-sided ideals of Theorem 7 would be zero ideals. Hence the $\mathrm{Zhu}$ algebras of $\mathrm{V}_{k_{u, v}}$ and $\mathrm{B}_{0 \mid 1}(u, v)$ would be isomorphic and every module over a $\mathrm{V}_{k}$ Zhu algebra would also be a module over the corresponding $\mathrm{B}_{0 \mid 1}(u, v)$ Zhu algebra. This would imply that every simple $\mathrm{V}_{k_{u, v}}$ module would also be a simple $\mathrm{B}_{0 \mid 1}(u, v)$ module and in particular that the field $\chi_{u, v}(z)$ acts trivially on every simple $\mathrm{V}_{k_{u, v}}$ module.

Consider the mode of $\chi_{u, v}(z)$ of index $-(u-1) v / 2$, that is, the coefficient of $z^{0}$. This mode acts non-trivially in $\mathrm{V}_{k_{u, v}}$ and therefore corresponds to a non-zero element in (a completion of) the universal enveloping algebras of $\widehat{\mathfrak{o s p}}(1 \mid 2)_{0}$, in particular its projection onto the universal enveloping algebra of the subalgebra $\widehat{\mathfrak{o s p}}(1 \mid 2)_{0}^{-} \oplus \widehat{\mathfrak{h}}$ is non-zero. So applying this mode to the highest weight vector of the Verma module Ind $\overline{\mathcal{B}}_{\lambda}^{+}$evaluates to a non-zero linear combination of monomials in $\widehat{\mathfrak{o s p}}(1 \mid 2)_{0}$ generators with non-positive index, where all occurrences of $h_{0}$ are replaced by $\lambda$, that is, the coefficients of the monomials will be polynomials in $\lambda$. These polynomials cannot vanish for every simple Verma module Ind $\overline{\mathcal{B}}_{\lambda}^{+}$, since there are infinitely many values of $\lambda$ for which Ind $\overline{\mathcal{B}}_{\lambda}^{+}$is simple. This contradicts 
$\chi_{u, v}(z)$ acting trivially on every simple $\mathrm{V}_{k_{u, v}}$ module, thus the image of the singular vector in the untwisted Zhu algebra must be non-zero.

The twisted case follows from the untwisted case. Since there are simple NeveuSchwarz Verma modules Ind $\overline{\mathcal{B}}_{\lambda}^{+}$which are not modules over $\mathrm{B}_{0 \mid 1}(u, v)$, the Ramond Verma module Ind $\overline{\mathcal{D}}_{k-\lambda}^{+} \cong \zeta\left(\right.$ Ind $\left.\overline{\mathcal{B}}_{\lambda}^{+}\right)$is also not a twisted module over $\mathrm{B}_{0 \mid 1}(u, v)$ and thus the image of the singular vector in the twisted Zhu algebras must be non-zero.

Recall that the Zhu algebras are filtered by conformal weight and so vectors in $\mathfrak{o s p}(1 \mid 2)$ such as $e$ and $h$ are assigned degree 1 in the Zhu algebras because they are the images of the conformal weight 1 fields $e(z)$ and $h(z)$. The conformal weight of the Virasoro field is 2 and its image in the untwisted and twisted Zhu algebras is proportional to the quadratic Casimirs $Q^{\mathfrak{o s p}}(1 \mid 2)$ and $Q$, respectively, which are therefore assigned degree 2. Finally, since the osp (1|2) quadratic Casimir, $Q^{\mathfrak{s} \mathfrak{p}}(1 \mid 2)$, is quadratic in the super-Casimir, $\Sigma$, the degree of $\Sigma$ in the untwisted Zhu algebra is 1 .

Lemma 9. Let $u \geq 2, v \geq 1$ be integers satisfying the condition (8). Then in the untwisted Zhu algebra of $\mathrm{V}_{k_{u, v}}$ the image of the singular vector is

$$
\left[\chi_{u, v}\right]= \begin{cases}e^{(u-1) / 2} g(h, \Sigma) & \text { if } u, v \text { odd } \\ e^{(u-2) / 2} x g(h, \Sigma) & \text { if } u, v \text { even }\end{cases}
$$

while in the twisted Zhu algebra it is

$$
\begin{aligned}
{\left[\chi_{u, v}\right]^{\tau}=e^{(u-1) / 2} g_{\tau}(h, Q) } & \text { if } u, v \text { odd }, \\
{\left[y_{0} \chi_{u, v}\right]^{\tau}=e^{(u-2) / 2} g_{\tau}(h, Q) } & \text { if } u, v \text { even },
\end{aligned}
$$

where $g$ and $g_{\tau}$ are polynomials whose degrees satisfy

$$
\operatorname{deg} g(h, \Sigma) \leq \frac{(u-1)(v-1)}{2}, \quad \operatorname{deg} g_{\tau}(h, Q) \leq \frac{(u-1)(v-1)+1}{2} .
$$

Proof. The $\mathfrak{o s p}(1 \mid 2)$ weight of the singular vector is $(u-1) \alpha$ and so the images of the singular vector in the untwisted and twisted Zhu algebras are elements of the universal enveloping algebras of $\mathfrak{o s p}(1 \mid 2)$ and $\mathfrak{s l}(2)$, respectively, of the same weight. Since $\mathfrak{o s p}(1 \mid 2)$ and $\mathfrak{s l}(2)$ are rank 1, it is clear that any homogeneous element of the universal enveloping algebras of positive weight can be written as the product of a monomial in the positive root vectors and an element of the centralisers of the Cartan subalgebra introduced in Theorem 1. The images of the singular must therefore be as given in (9) and (10).

Since the conformal weight of the singular vectors is $(u-1) v / 2$, the total degree of the images of the singular vectors is at most $(u-1) v / 2$. The factors of $e$ and $x$ in the images of the singular vectors each contribute 1 unit of degree and the right-hand sides of the inequalities of (11) are just the upper bounds on how many units of degree the polynomials $g$ and $g_{\tau}$ can contribute. 
Definition 7. The Kac table of $\mathrm{B}_{0 \mid 1}(u, v)$ is the set of pairs of integers

$$
\mathrm{K}_{u, v}=\{(i, j): 1 \leq i \leq u-1,1 \leq j \leq v-1\}
$$

and the Neveu-Schwarz and Ramond Kac tables are the subsets

$$
\begin{aligned}
& \mathrm{K}_{u, v}^{\mathrm{NS}}=\left\{(i, j) \in \mathrm{K}_{u, v}: i+j \text { is odd }\right\}, \\
& \mathrm{K}_{u, v}^{\mathrm{R}}=\left\{(i, j) \in \mathrm{K}_{u, v}: i+j \text { is even }\right\},
\end{aligned}
$$

respectively. Let $\sim$ denote the equivalence relation on $\mathrm{K}_{u, v}$ given by $(i, j) \sim\left(i^{\prime}, j^{\prime}\right)$ if and only if $(i, j)=\left(i^{\prime}, j^{\prime}\right)$ or $(i, j)=\left(u-i^{\prime}, v-j^{\prime}\right)$. The reduced Kac table, the reduced Neveu-Schwarz Kac table and the reduced Ramond Kac table are then respectively defined to be

$$
\overline{\mathrm{K}}_{u, v}=\mathrm{K}_{u, v} / \sim, \quad \overline{\mathrm{K}}_{u, v}^{\mathrm{NS}}=\mathrm{K}_{u, v}^{\mathrm{NS}} / \sim, \quad \overline{\mathrm{K}}_{u, v}^{\mathrm{R}}=\mathrm{K}_{u, v}^{\mathrm{R}} / \sim .
$$

For $i, j \in \mathbb{Z}$, let

$$
\lambda_{i, j}=\frac{i-1}{2}-\frac{1+(-1)^{i+j}}{4}-\frac{u}{2 v} j, \quad s_{i, j}=\frac{i}{2}-\frac{u}{2 v} j, \quad q_{i, j}=\frac{(u j-v i)^{2}-4 v^{2}}{8 v^{2}} .
$$

Theorem 10. Let $u \geq 2, v \geq 1$ be integers satisfying the condition (8). Up to normalisation, the images of the singular vectors $\chi_{u, v}$ in $\mathrm{V}_{k_{u, v}}$ are given by the following formulae: In the untwisted Zhu algebra,

$$
\left[\chi_{u, v}\right]= \begin{cases}e^{(u-1) / 2} g(\Sigma) & u, v \text { odd } \\ e^{(u-2) / 2} x g(\Sigma) & u, v \text { even }\end{cases}
$$

and in the twisted Zhu algebra,

$$
e^{(u-2) / 2} g_{\tau}(Q)= \begin{cases}{\left[\chi_{u, v}\right]^{\tau}} & u, v \text { odd } \\ {\left[y_{0} \chi_{u, v}\right]^{\tau}} & u, v \text { even }\end{cases}
$$

where

$$
g(\Sigma)=\prod_{(i, j) \in \mathrm{K}_{u, v}^{\mathrm{NS}}}\left(\Sigma-s_{i, j}\right) \quad \text { and } \quad g_{\tau}(Q)=\prod_{[(i, j)] \in \overline{\mathrm{K}}_{u, v}^{\mathrm{R}}}\left(Q-q_{i, j}\right) .
$$

The proof of the above polynomial formulae is where most of the effort of this paper is spent. The main idea is to evaluate the action of the zero mode of the singular vector on candidate relaxed highest weight vectors. Since the images of the singular vector in the Zhu algebras are polynomials in $\mathfrak{o s p}(1 \mid 2)$ or $\mathfrak{s l}(2)$ generators, these polynomials can be determined through sufficiently many evaluations. Unfortunately, closed formulae for singular vectors are notoriously hard to find. We sidestep this issue by constructing the singular vector $\chi_{u, v}$ within free field realisations as the image of screening operators. However, these free field methods require significant preparation and so, for greater clarity of presentation, we postpone the proof of Theorem 10 to Section 4 where it has been split up into four cases: Theorems 25, 26, 28 and 29. 
Theorem 11. Let $u \geq 2, v \geq 1$ be integers satisfying the condition (8). The minimal model $\mathfrak{o s p}(1 \mid 2)$ vertex operator superalgebra $\mathrm{B}_{0 \mid 1}(u, v)$ is rational in category $\mathscr{O}$ in both the Neveu-Schwarz and Ramond sectors, that is, both sectors admit only a finite number of isomorphism classes of simple modules and every module is semisimple. Any simple $\mathrm{B}_{0 \mid 1}(u, v)$ module in category $\mathscr{R}$ is isomorphic to one of the following mutually inequivalent modules.

In the Neveu-Schwarz sector:

(1) $\mathcal{A}_{\lambda_{i, 0}}$ or $\Pi \mathcal{A}_{\lambda_{i, 0}}$, where $1 \leq i \leq u-1$ and $i$ is odd.

(2) $\mathcal{B}_{\lambda_{i, j}}^{+}$or $\Pi \mathcal{B}_{\lambda_{i, j}}^{+}$, where $(i, j) \in \mathrm{K}_{u, v}^{\mathrm{NS}}$.

(3) $\mathcal{B}_{-\lambda_{i, j}}^{-}$or $\Pi \mathcal{B}_{-\lambda_{i, j}}^{-}$, where $(i, j) \in \mathrm{K}_{u, v}^{\mathrm{NS}}$.

(4) $\mathcal{C}_{[\lambda], s_{i, j}}$, where $[\lambda] \in \mathbb{C} / 2 \mathbb{Z},(i, j) \in \mathrm{K}_{u, v}^{\mathrm{NS}}$ and $s_{i, j}^{2} \neq(\mu+1 / 2)^{2}$ for all $\mu \in$ $[\lambda] \cup[\lambda+1]$.

In the Ramond sector:

(5) $\mathcal{F}_{\lambda_{i, 0}}$ or $\Pi \mathcal{F}_{\lambda_{i, 0}}$, where $1 \leq i \leq u-1$ and $i$ is even.

(6) $\mathcal{D}_{\lambda_{i, j}}^{+}$or $\Pi \mathcal{D}_{\lambda_{i, j}}^{+}$, where $(i, j) \in \mathrm{K}_{u, v}^{\mathrm{R}}$.

(7) $\mathcal{D}_{-\lambda_{i, j}}^{-}$or $\Pi \mathcal{D}_{-\lambda_{i, j}}^{-}$, where $(i, j) \in \mathrm{K}_{u, v}^{\mathrm{R}}$.

(8) $\mathcal{E}_{[\lambda], q_{i, j}}$ or $\Pi \mathcal{E}_{[\lambda], q_{i, j}}$, where $[\lambda] \in \mathbb{C} / 2 \mathbb{Z},[(i, j)] \in \overline{\mathrm{K}}_{u, v}^{\mathrm{R}}$ and $q_{i, j} \neq \mu(\mu+2)$ for all $\mu \in[\lambda]$.

Note that the parity reversals of the dense modules $\mathcal{C}_{[\lambda], s_{i, j}}$ do not need to be included, as $\Pi \mathcal{C}_{[\lambda], s_{i, j}} \cong \mathcal{C}_{[\lambda+1],-s_{i, j}}=\mathcal{C}_{[\lambda+1], s_{u-i, v-j}}$.

Proof. The theorem follows by evaluating the action of the image of the singular vector in the untwisted and twisted Zhu algebras on the simple modules of Theorems 2 and 3 . Those simple $\mathfrak{o s p}(1 \mid 2)$ and $\mathfrak{s l}(2)$ modules on which the image of the singular vector acts trivially can be induced to modules over $\mathrm{B}_{0 \mid 1}(u, v)$.

In both the Neveu-Schwarz and Ramond sectors, the factors of $e$ and $x$ in the image of the singular vectors cannot act trivially on infinite-dimensional simple weight modules. They do however act trivially on finite-dimensional simple weight modules up to a certain dimension, that is, those of items (1) and (5).

For the infinite-dimensional simple weight modules, since the factors of $e$ and $x$ cannot act trivially, the polynomials $g$ or $g_{\tau}$ must. Thus, in the Neveu-Schwarz sector the super-Casimir $\Sigma$ must act as $\pm s_{i, j} \cdot$ id for one of the $s_{i, j}$ in the factorisation (12) of $g$. The modules for which this is the case are precisely those of items (2)-(4). Similarly, in the Ramond sector the $\mathfrak{s l}(2)$ Casimir $Q$ must act as $q_{i, j} \cdot$ id for one of the $q_{i, j}$ in the factorisation (12) of $g_{\tau}$. The modules for which this is the case are precisely those of items (6)-(8).

To conclude rationality in category $\mathscr{O}$ one must show that there exist no indecomposable extensions of modules in items (1) and (2) or modules in items (5) and (6). Consider first the Neveu-Schwarz sector. An indecomposable self-extension would have as its space of relaxed highest weight vectors an indecomposable selfextension $\overline{\mathcal{M}}$ of a simple highest weight Zhu algebra module of highest weight $\lambda \alpha$. Further, the weight space $\overline{\mathcal{M}}_{\lambda \alpha}$ of $\overline{\mathcal{M}}$ of weight $\lambda \alpha$ would be a two-dimensional module over $\mathbb{C}[h, \Sigma]$. If $\overline{\mathcal{M}}_{\lambda \alpha}$ were semisimple it would generate two simple direct summands of $\overline{\mathcal{M}}$ under the action of the Zhu algebra contradicting the indecomposability of $\overline{\mathcal{M}}$. Thus $\overline{\mathcal{M}}_{\lambda \alpha}$ would be indecomposable over $\mathbb{C}[h, \Sigma]$. If we choose 
a Poincaré-Birkhoff-Witt ordering which places $y$ to the left of $x$, then $\Sigma=$ $h-2 y x+1 / 2$. By Definition $3, h$ acts semisimply in category $\mathscr{O}$ and $y x$ acts trivially on the weight space of weight $\lambda \alpha$, because both composition factors of $\overline{\mathcal{M}}$ contain no vectors of weight $(\lambda+1) \alpha$. Thus, the formula for $\Sigma$ implies that $\Sigma$ acts semisimply on the weight space $\overline{\mathcal{M}}_{\lambda \alpha}$, thereby contradicting indecomposability. To rule out indecomposable extensions of two inequivalent simple modules, $\mathcal{M}$ and $\mathcal{N}$, in category $\mathscr{O}$, note that either the indecomposable module or its contragredient dual would be a highest weight module and thus a quotient of a Verma module. In particular the highest weight of the submodule would need to match the weight of a singular vector of the Verma module. However, from [46, Thm. 3.1], it is easy to verify that Verma modules with highest weights as in items (1) or (2) do not have singular vectors with weights as in items (1) or (2).

The rationality of the Ramond sector in category $\mathscr{O}$ follows from that of the Neveu-Schwarz sector after twisting by the algebra isomorphism $\zeta$ of (6): Since $\zeta$ preserves the triangular decomposition used to define category $\mathscr{O}$, the twist of an indecomposable extension of Ramond modules in category $\mathscr{O}$ would be an indecomposable extension of Neveu-Schwarz modules in category $\mathscr{O}$, but no such extensions exist in the Neveu-Schwarz sector of category $\mathscr{O}$ and so neither can they in the Ramond sector of category $\mathscr{O}$.

\section{Free field algebras and screening}

In this section we shall set the stage for proving Theorem 10 by following a line of reasoning that has already been proved to be very successful for similar Zhu algebra related problems [32], [33], [25], [34]. The basic idea is as follows. First we construct a free field realisation of the universal $\mathfrak{o s p}(1 \mid 2)$ vertex operator superalgebra $\bigvee_{k}$, that is, we embed $\bigvee_{k}$ into free field vertex operator superalgebras. Free field vertex operator superalgebras have the convenient property of allowing one to easily construct certain module homomorphisms called screening operators which in turn can be used to realise the singular vector as the image of certain highest weight vectors. Armed with these formulae for singular vectors, we then evaluate the action of the zero mode of the singular vector on candidate relaxed highest weight vectors to deduce the image of the singular vector in the Zhu algebras.

\section{The Heisenberg free field algebra and lattice vertex algebras}

The rank $r$ Heisenberg algebra is the affinisation of the trivial $r$ dimensional complex Lie algebra. Though the only ranks required here will be $r=1$ and $r=2$, we give the general definition for conceptual clarity. The definition presented here is essentially the definition of "free (super) bosons" in [47]. An exhaustive description of lattice vertex algebras is given in [48] and all results of this subsection are a specialisation of those of [48].

Let $\mathcal{H}_{r}$ be an $r$-dimensional complex (purely even) vector space together with a symmetric non-degenerate bilinear form $(-,-)$ (recall that all such forms are equivalent over the complex numbers). The Heisenberg algebra is given by

$$
\mathfrak{H}_{r}=\mathcal{H}_{r} \otimes \mathbb{C}\left[t, t^{-1}\right] \oplus \mathbb{C} \mathbf{1},
$$


as a vector space, where $\mathbf{1}$ is the central element which will always be identified with the identity when acting on modules. The Lie bracket is given by

$$
\left[a_{m}, b_{n}\right]=m(a, b) \delta_{m+n, 0} \mathbf{1}, \quad a_{m}=a \otimes t^{m}, \quad b_{n}=b \otimes t^{n}, \quad a, b \in \mathcal{H}_{r} .
$$

The usual triangular decomposition of the Heisenberg algebra is then given by

$$
\mathfrak{H}_{r}^{ \pm}=\mathcal{H}_{r} \otimes \mathbb{C}\left[t^{ \pm}\right] t^{ \pm}, \quad \mathfrak{H}_{r}^{0}=\mathcal{H}_{r} \oplus \mathbb{C} \mathbf{1} .
$$

The Verma modules with respect to this decomposition are called Fock spaces and they are all simple. Writing $\mathfrak{H}_{r}^{\geq}=\mathfrak{H}_{r}^{+} \oplus \mathfrak{H}_{r}^{0}$, we define

$$
\mathbb{F}_{b}=\operatorname{Ind}_{\mathfrak{H}_{r}}^{\mathfrak{H}_{r}} \mathbb{C}|b\rangle, \quad b \in \mathcal{H}_{r},
$$

to be the Verma module induced from the 1-dimensional $\mathfrak{H}_{r}^{\geq}$module characterised by

$$
a_{0}|b\rangle=(a, b)|b\rangle, \quad \mathbf{1}|b\rangle=|b\rangle, \quad \mathfrak{H}_{r}^{+}|b\rangle=0 .
$$

Definition 8. Let $\mathcal{H}_{r}$ be an $r$-dimensional complex vector space with any choice of symmetric non-degenerate bilinear form $(-,-)$ and any choice of basis $\left\{a^{1}, \ldots, a^{r}\right\}$. The rank $r$ Heisenberg vertex algebra is the unique vertex algebra that is strongly generated by fields $a^{1}(z), \ldots, a^{r}(z)$ (one for each basis vector), subject to the defining operator product expansions

$$
a^{i}(z) a^{j}(z) \sim \frac{\left(a^{i}, a^{j}\right)}{(z-w)^{2}}, \quad 1 \leq i, j \leq r,
$$

and satisfies no additional relations beyond those required by vertex algebra axioms.

The operator product expansions (8) imply that the modes of the Laurent expansions

$$
a(z)=\sum_{n \in \mathbb{Z}} a_{n} z^{-n-1}, \quad a \in \mathcal{H}_{r}
$$

satisfy the commutation relations (13) of the Heisenberg algebra $\mathfrak{H}_{r}$. As a module over itself $\mathrm{H}_{r}$ corresponds to $\mathbb{F}_{0}$. The Heisenberg vertex algebras admit continuous families of conformal structures, however, we shall wait until later applications to choose a specific one.

Vertex operators are maps between Fock spaces whose definition requires us to extend the universal enveloping algebra of the Heisenberg Lie algebra $\mathbf{U}\left(\mathfrak{H}_{r}\right)$ by the group algebra $\mathbb{C}\left[\mathcal{H}_{r}\right]$, where $\mathcal{H}_{r}$ is viewed as an abelian group under vector space addition. For any vector $a \in \mathcal{H}_{r}$, we denote the corresponding group algebra element by $\mathrm{e}^{a}$. Then $\mathbb{C}\left[\mathcal{H}_{r}\right] \otimes \mathbf{U}\left(\mathfrak{H}_{r}\right)$ is an associative algebra after imposing the relations

$$
\left[a_{n}, \mathrm{e}^{b}\right]=(a, b) \delta_{n, 0} \mathrm{e}^{b}, \quad a, b \in \mathcal{H}, n \in \mathbb{Z} .
$$

Finally, we define the group algebra elements to act on the highest weight vectors of Fock spaces in the following way:

$$
\mathrm{e}^{a}|b\rangle=|a+b\rangle
$$


For any $a \in \mathcal{H}_{r}$, the vertex operator $\mathrm{V}_{a}(z)$, corresponding to $a$, is given by

$$
\mathrm{V}_{a}(z)=\mathrm{e}^{a} z^{a_{0}} \prod_{m \geq 1} \exp \left(\frac{a_{-m}}{m} z^{m}\right) \exp \left(-\frac{a_{m}}{m} z^{-m}\right) .
$$

On Fock spaces $\mathrm{V}_{a}(z)$ defines a map

$$
\mathrm{V}_{a}(z): \mathbb{F}_{b} \mapsto \mathbb{F}_{a+b} \llbracket z, z^{-1} \rrbracket z^{(a, b)}
$$

and the composition of $\ell$ vertex operators associated to vectors $a^{1}, \ldots, a^{\ell} \in \mathcal{H}_{r}$ is given by

$$
\begin{aligned}
& \mathrm{V}_{a^{1}}\left(z_{1}\right) \cdots \mathrm{V}_{a^{\ell}}\left(z_{\ell}\right) \\
& =\prod_{1 \leq i<j \leq \ell}\left(z_{i}-z_{j}\right)^{\left(a^{i}, a^{j}\right)} \mathrm{e}^{a^{1}+\cdots+a^{\ell}} \prod_{i=1}^{\ell} z_{i}^{a_{0}^{i}} \prod_{m \geq 1} \exp \left(\sum_{i=1}^{\ell} \frac{a_{-m}^{i}}{m} z_{i}^{m}\right) \exp \left(-\sum_{i=1}^{\ell} \frac{a_{m}^{i}}{m} z_{i}^{-m}\right) .
\end{aligned}
$$

Lattice vertex algebras are (infinite order) extensions of Heisenberg vertex algebras $\mathrm{H}_{r}$ constructed by picking linearly independent vectors $a^{1}, \ldots, a^{m}$ in $\mathcal{H}_{r}$ such that the pairings of these vectors are integral. Let $\left\langle a^{1}, \ldots, a^{m}\right\rangle=\bigoplus_{i=1}^{m} \mathbb{Z} a^{i}$ denote the lattice in $\mathcal{H}_{r}$ generated by $a^{1}, \ldots, a^{m}$.

Definition 9. Let $\mathcal{H}_{r}$ be an $r$-dimensional complex vector space with a choice of symmetric non-degenerate bilinear form $(-,-)$ and associated Heisenberg vertex algebra $\mathrm{H}_{r}$, further let $\left\{a^{1}, \ldots, a^{m}\right\}, 1 \leq m \leq r$ be a choice of linearly independent vectors in $\mathcal{H}_{r}$, such that the pairings of these vectors are integral. The lattice vertex algebra $\mathrm{L}_{\left\langle a^{1}, \ldots, a^{m}\right\rangle}$ is the extension of $\mathrm{H}_{r} \cong \mathbb{F}_{0}$ which as a vector space is given by

$$
\mathrm{L}_{\left\langle a^{1}, \ldots, a^{m}\right\rangle}=\bigoplus_{\mu \in\left\langle a^{1}, \ldots, a^{m}\right\rangle} \mathbb{F}_{\mu}=\bigoplus_{n_{1}, \ldots, n_{m} \in \mathbb{Z}} \mathbb{F}_{n_{1} a^{1}+\cdots+n_{m} a^{m}},
$$

where the state-field correspondence is uniquely determined by assigning to each highest weight vector $\left|n_{1} a^{1}+\cdots+n_{m} a^{m}\right\rangle$ the vertex operator $\mathrm{V}_{n_{1} a^{1}+\cdots n_{m} a^{m}}(z)$.

Remark 2. Note that we are not requiring the pairing on the lattice in the above definition to be even or positive definite, as it will be necessary to consider indefinite lattices for the free field realisation of the $\beta \gamma$ ghost vertex algebra constructed below.

Proposition 12. Let $\left\langle a^{1}, \ldots, a^{m}\right\rangle^{*}=\left\{\mu \in \mathcal{H}_{r}:(\mu, \lambda) \in \mathbb{Z} \forall \lambda \in\left\langle a^{1}, \ldots, a^{m}\right\rangle\right\}$ be the dual of $\left\langle a^{1}, \ldots, a^{m}\right\rangle$ in $\mathcal{H}_{r}$. For each coset $[\lambda] \in\left\langle a^{1}, \ldots, a^{m}\right\rangle^{*} /\left\langle a^{1}, \ldots, a^{m}\right\rangle$,

$$
\mathbb{F}_{[\lambda]}=\bigoplus_{\mu \in\left\langle a^{1}, \ldots, a^{m}\right\rangle} \mathbb{F}_{\lambda+\mu}
$$

is a module over $\mathrm{L}_{\left\langle a^{1}, \ldots, a^{m}\right\rangle}$.

Proof. Clearly the action of $\mathrm{L}_{\left\langle a^{1}, \ldots, a^{m}\right\rangle}$ closes on $\mathbb{F}_{[\lambda]}$, so the only potential obstruction to $\mathbb{F}_{[\lambda]}$ being a module (and not a twisted module) is the fields of $\mathrm{L}_{\left\langle a^{1}, \ldots, a^{m}\right\rangle}$ being single valued, that is, that their series expansions only have integer exponents. This is true if and only if it is true for the vertex operators $\mathrm{V}_{a^{i}}(z), 1 \leq i \leq m$ and it is true for those by construction due to the Fock space weights all lying in $\left\langle a^{1}, \ldots, a^{m}\right\rangle^{*}$. 


\section{The $\beta \gamma$ ghosts}

We refer to [41] for an in depth treatment of the $\beta \gamma$ ghosts. The $\beta \gamma$ ghost algebra is an infinite-dimensional complex Lie algebra

$$
\mathfrak{G}_{\beta \gamma}=\bigoplus_{n \in \mathbb{Z}} \mathbb{C} \beta_{n} \oplus \bigoplus_{n \in \mathbb{Z}} \mathbb{C} \gamma_{n} \oplus \mathbb{C} 1
$$

whose Lie brackets are

$$
\left[\gamma_{m}, \beta_{n}\right]=\delta_{m+n, 0} \mathbf{1}, \quad\left[\beta_{m}, \beta_{n}\right]=\left[\gamma_{m}, \gamma_{n}\right]=0, \quad m, n \in \mathbb{Z} .
$$

The $\beta \gamma$ ghost algebra admits the relaxed triangular decomposition

$$
\mathfrak{G}_{\beta \gamma}^{ \pm}=\bigoplus_{n \geq 1} \mathbb{C} \beta_{ \pm n} \oplus \bigoplus_{n \geq 1} \mathbb{C} \gamma_{ \pm n}, \quad \mathfrak{G}_{\beta \gamma}^{0}=\mathbb{C} \beta_{0} \oplus \mathbb{C} \gamma_{0} \oplus \mathbb{C} \mathbf{1}
$$

Conveniently, $\mathfrak{G}_{\beta \gamma}^{0}$ is just the $A_{1}$ Weyl algebra for which Block classified all simple modules [39]. Here we shall only need simple weight modules, and to list them we introduce the operator $J=\gamma_{0} \beta_{0}$.

Proposition 13. Every simple $\mathfrak{G}_{\beta \gamma}^{0}$ weight module is isomorphic to one of the following.

(1) The simple highest weight module $\overline{\mathcal{G}}^{+}=\mathbb{C}\left[\gamma_{0}\right] \bar{\Omega}$ generated by a highestweight vector $\bar{\Omega}$ satisfying $\beta_{0} \bar{\Omega}=0$, hence $J \bar{\Omega}=0$.

(2) The simple lowest weight $\mathfrak{G}_{\beta \gamma}^{0}$-module $\overline{\mathcal{G}}^{-}=\mathbb{C}\left[\beta_{0}\right] \bar{\omega}$ generated by a lowestweight vector $\bar{\omega}$ satisfying $\gamma_{0} \bar{\omega}=0$, hence $J \bar{\omega}=\bar{\omega}$.

(3) The simple dense module $\overline{\mathcal{W}}_{[\lambda]},[\lambda] \in \mathbb{C} / \mathbb{Z},[\lambda] \neq[0]$ with a basis of weight $\bar{u}_{j}$, satisfying $J \bar{u}_{j}=j \bar{u}_{j}$, hence $\overline{\mathcal{W}}_{[\lambda]}=\mathbb{C}\left[\beta_{0}\right] \bar{u}_{\mu} \oplus \mathbb{C}\left[\gamma_{0}\right] \gamma_{0} \bar{u}_{\mu}$.

In addition to the simple weight modules above, we also need to consider two indecomposable weight modules whose $J$-weight support is $\mathbb{Z}$ and whose isomorphism classes are determined by the following short exact sequences $\left(\operatorname{Ext}^{1}\left(\overline{\mathcal{G}}^{-}, \overline{\mathcal{G}}^{+}\right)=\operatorname{Ext}^{1}\left(\overline{\mathcal{G}}^{+}, \overline{\mathcal{G}}^{-}\right)=\mathbb{C}\right)$ :

$$
0 \rightarrow \overline{\mathcal{G}}^{+} \rightarrow \overline{\mathcal{W}}_{0}^{+} \rightarrow \overline{\mathcal{G}}^{-} \rightarrow 0, \quad 0 \rightarrow \overline{\mathcal{G}}^{-} \rightarrow \overline{\mathcal{W}}_{0}^{-} \rightarrow \overline{\mathcal{G}}^{+} \rightarrow 0
$$

Both may be realised on the space $\mathbb{C}\left[\gamma_{0}\right] \bar{u}_{0} \oplus \mathbb{C}\left[\beta_{0}\right] \bar{u}_{1}$, where $\beta_{0} \bar{u}_{0}=0$ and $\gamma_{0} \bar{u}_{1}=$ $a^{+} \bar{u}_{0}$, for $\mathcal{W}_{0}^{+}$, and $\beta_{0} \bar{u}_{0}=a^{-} \bar{u}_{1}$ and $\gamma_{0} \bar{u}_{1}=0$, for $\mathcal{W}_{0}^{-}$. We may normalise the basis vectors so that $a^{+}=a^{-}=1$.

We denote the inductions of the above modules by the same symbols but without the overlines:

$$
\begin{aligned}
& \mathcal{G}^{+}=\operatorname{Ind}_{\mathfrak{G} \frac{\bar{\beta} \gamma}{\bar{\beta} \gamma}}^{\mathfrak{G}_{\beta \gamma}} \overline{\mathcal{G}}^{+}, \quad \mathcal{G}^{-}=\operatorname{Ind}_{\mathfrak{G} \frac{\bar{\beta} \gamma}{\mathfrak{G}_{\beta \gamma}}}^{\mathfrak{G}_{\mathcal{G}}} \overline{\mathcal{G}}^{-},
\end{aligned}
$$

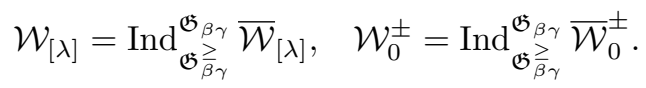


The first three of the above modules are simple while the last are characterised by the non-split exact sequences

$$
0 \rightarrow \mathcal{G}^{+} \rightarrow \mathcal{W}_{0}^{+} \rightarrow \mathcal{G}^{-} \rightarrow 0, \quad 0 \rightarrow \mathcal{G}^{-} \rightarrow \mathcal{W}_{0}^{-} \rightarrow \mathcal{G}^{+} \rightarrow 0 .
$$

The $\beta \gamma$ algebra admits an algebra automorphism $\sigma$ called spectral flow whose action on generators is given by

$$
\sigma\left(\beta_{n}\right)=\beta_{n-1}, \quad \sigma\left(\gamma_{n}\right)=\gamma_{n+1}, \quad \sigma(\mathbf{1})=\mathbf{1} .
$$

Since spectral flow is an algebra automorphism, it can be used to twist modules (recall Definition 5). In particular, $\mathcal{G}^{+}$and $\mathcal{G}^{-}$are related by spectral flow:

$$
\mathcal{G}^{-} \cong \sigma^{-1} \mathcal{G}^{+} \text {. }
$$

Note, however, that spectral flow does not preserve the relaxed triangular decomposition (16) and therefore the spectral flow of a relaxed highest weight module will generally no longer be relaxed highest weight (it will instead be relaxed highest weight with respect to a different triangular decomposition).

Definition 10. The $\beta \gamma$ ghost vertex algebra $\mathrm{G}$ is the unique vertex algebra that is strongly generated by two even fields $\beta(z)$ and $\gamma(z)$, has the operator product expansions

$$
\gamma(z) \beta(w) \sim \beta(w) \gamma(z) \sim \frac{1}{z-w}, \quad \beta(z) \beta(w) \sim \gamma(z) \gamma(w) \sim 0 .
$$

and satisfies no additional relations beyond those required by vertex algebra axioms.

The operator product expansion (17) implies that the modes of the Laurent expansion

$$
\beta(z)=\sum_{n \in \mathbb{Z}} \beta_{n} z^{-n-1}, \quad \gamma(z)=\sum_{n \in \mathbb{Z}} \gamma_{n} z^{-n}
$$

satisfy the commutation relations (15) of the $\beta \gamma$ ghost algebra. As a $\beta \gamma$ ghost algebra module, $\mathrm{G}$ is isomorphic to $\mathcal{G}^{+}$. The $\beta \gamma$ ghost vertex algebra admits a 1parameter family of Virasoro fields. Here we shall only consider the unique choice $T=-: \beta(z) \partial \gamma(z)$ : which assigns conformal weight 1 to $\beta$ and conformal weight 0 to $\gamma$. Below it will also be necessary to consider the field $J(z)=: \beta(z) \gamma(z)$ : which generates a rank 1 Heisenberg vertex algebra and grades the $\beta \gamma$ ghost vertex algebra by assigning weight 1 to $\beta$ and weight -1 to $\gamma$. The central charge for this choice of Virasoro field is $c=2$.

The $\beta \gamma$ ghost vertex algebra admits a free field realisation in terms of a lattice algebra, that is, there exists an embedding from $\mathrm{G}$ to a lattice algebra.

Proposition 14. Let $\mathrm{H}_{2}$ be the rank 2 Heisenberg vertex algebra and let $\theta, \psi$ be a basis of $\mathcal{H}_{2}$ such that the non-vanishing pairings of $(-,-)$ are $(\psi, \psi)=-(\theta, \theta)=1$. Then there exists an embedding $\mathrm{G} \rightarrow \mathrm{L}_{\langle\theta+\psi\rangle}$ of vertex algebras defined by

$$
\beta(z) \mapsto \mathrm{V}_{\theta+\psi}(z), \quad \gamma(z) \mapsto: \psi(z) \mathrm{V}_{-\theta-\psi}(z):
$$


Proof. The map is a homomorphism of vertex algebras because $\mathrm{V}_{\theta+\psi}(z)$ and $: \psi(z) \mathrm{V}_{-\theta-\psi}(z)$ : satisfy the same operator product expansions as $\beta(z)$ and $\gamma(z)$ do. It is injective because it is non-trivial and the $\beta \gamma$ ghost vertex algebra is simple.

Since $G$ is a vertex subalgebra of $L_{\langle\theta+\psi\rangle}$, we can decompose $L_{\langle\theta+\psi\rangle}$ modules into $\mathrm{G}$ modules. Before we do so, note that the dual lattice of $\langle\theta+\psi\rangle$ can be written as

$$
\langle\theta+\psi\rangle^{*}=\{\lambda(\theta+\psi)+n \psi: \lambda \in \mathbb{C}, n \in \mathbb{Z}\} .
$$

Proposition 15. As modules over $\mathrm{G}$, the $\mathrm{L}_{\langle\theta+\psi\rangle}$ modules of Theorem 12 decompose as

$$
\begin{aligned}
& \mathbb{F}_{[n \psi]} \cong \sigma^{n+1} \mathcal{W}_{0}^{-} \quad \text { for } n \in \mathbb{Z}, \\
& \mathbb{F}_{[\lambda(\theta+\psi)+n \psi]} \cong \sigma^{n+1} \mathcal{W}_{[\lambda]} \quad \text { for } \lambda \in \mathbb{C} \backslash \mathbb{Z}, n \in \mathbb{Z}
\end{aligned}
$$

Proof. It was shown in [41] that the characters of $\sigma^{n+1} \mathcal{W}_{0}^{-}$and $\sigma^{n+1} \mathcal{W}_{[\lambda]}$, with $[\lambda] \in \mathbb{Z} / \mathbb{Z},[\lambda] \neq[0]$ and $n \in \mathbb{Z}$ form a basis of the span of characters of relaxed highest weight modules and their spectral flow twists. Thus, the proposition follows by comparing characters of the left and right-hand sides of (19). The embedding (18) implies that

$$
\begin{aligned}
& J(z)=: \beta(z) \gamma(z): \mapsto-\theta(z) \\
& T(z)=-: \beta(z) \partial \gamma(z): \mapsto \frac{1}{2}: \psi^{2}(z):-\frac{1}{2}: \theta^{2}(z):-\frac{1}{2} \partial(\psi(z)-\theta(z)) .
\end{aligned}
$$

Thus

$$
\begin{aligned}
\operatorname{tr}_{\mathbb{F}_{[\lambda(\theta+\psi)+n \psi]}} & \left(z^{J_{0}} q^{L_{0}-1 / 12}\right) \\
& =\sum_{m \in \mathbb{Z}} \operatorname{tr}_{\mathbb{F}_{(\lambda+m)(\theta+\psi)+n \psi}}\left(z^{J_{0}} q^{L_{0}-1 / 12}\right)=\sum_{m \in \mathbb{Z}} \frac{z^{\lambda+m} q^{(n+1)(2(\lambda+m)+n) / 2}}{\eta(q)^{2}} \\
& =\frac{z^{\lambda} q^{(n+1) \lambda+(n+1) n / 2}}{\eta(q)^{2}} \sum_{m \in \mathbb{Z}} z^{m} q^{(n+1) m}=\operatorname{ch}\left[\sigma^{n+1} \mathcal{W}_{\lambda}\right],
\end{aligned}
$$

where the last equality was taken from Equation (5.1) of [41]. This proves the proposition whenever the right-hand side of (19) is simple, that is, when $\lambda$ is not an integer. For the non-simple case the character implies 3 possibilities: the righthand side is either semisimple or isomorphic to either $\sigma^{n+1} \mathcal{W}_{0}^{+}$or $\sigma^{n+1} \mathcal{W}_{0}^{-}$. That the right-hand side is isomorphic to $\sigma^{n+1} \mathcal{W}_{0}^{-}$then follows by checking the action of the $\beta$ and $\gamma$ fields on the highest weight vectors of the Fock space summands in $\mathbb{F}_{[n \psi]}$.

\section{The $b c$ ghosts}

The final free field algebra which we shall consider is the $b c$ ghost algebra, the fermionic analogue of the $\beta \gamma$ ghosts. The $b c$ ghost algebras are a pair of infinitedimensional Lie superalgebras

$$
\mathfrak{b} \mathfrak{c}_{\epsilon}=\bigoplus_{n \in \mathbb{Z}+\epsilon} \mathbb{C} b_{n} \oplus \bigoplus_{n \in \mathbb{Z}+\epsilon} \mathbb{C} \gamma_{n} \oplus \mathbb{C} \mathbf{1}, \quad \epsilon=0, \frac{1}{2},
$$


whose Lie brackets are

$$
\left\{b_{m}, c_{n}\right\}=\delta_{m+n, 0}, \quad\left\{b_{m}, b_{n}\right\}=\left\{c_{m}, c_{n}\right\}=0, \quad m, n \in \mathbb{Z}+\epsilon .
$$

As in the previous sections on $\mathfrak{o s p}(1 \mid 2), \epsilon=0$ corresponds to the Neveu-Schwarz sector and $\epsilon=1 / 2$ corresponds to the Ramond sector.

The $b c$ ghost algebra $\mathfrak{b c}_{\epsilon}$ admits a triangular decomposition

$$
\mathfrak{b} \mathfrak{c}_{\epsilon}^{ \pm}=\bigoplus_{n \geq 1} b_{ \pm(n-\epsilon)} \oplus \bigoplus_{n \geq 1} c_{ \pm(n-\epsilon)}, \quad \mathfrak{b} \mathfrak{c}_{0}^{0}=\mathbb{C} b_{0} \oplus \mathbb{C} c_{0} \oplus \mathbb{C} \mathbf{1}, \quad \mathfrak{b} \mathfrak{c}_{1 / 2}^{0}=\mathbb{C} \mathbf{1}
$$

Up to isomorphism and parity reversal there is only one simple $\mathfrak{b} \mathfrak{c}_{0}^{\geq}=\mathfrak{b} \mathfrak{c}_{0}^{0} \oplus \mathfrak{b} \mathfrak{c}_{0}^{+}$ module $\mathbb{C}|\mathrm{NS}\rangle \oplus \mathbb{C} c_{0}|\mathrm{NS}\rangle$, on which $\mathbf{1}$ acts as the identity and $\mathfrak{b c}_{0}^{+}$acts trivially. The action of $b_{0}$ satisfies $b_{0}|\mathrm{NS}\rangle=0$ (this is necessary for simplicity) and the global parity of the module is fixed by assigning even parity to $|\mathrm{NS}\rangle$. The Verma module,

$$
\mathbb{F}^{\mathrm{NS}}=\operatorname{Ind}_{\mathfrak{b c}_{0}^{\geq}}^{\mathfrak{b} \mathfrak{c}_{0}}\left(\mathbb{C}|\mathrm{NS}\rangle \oplus \mathbb{C} c_{0}|\mathrm{NS}\rangle\right)
$$

together with its parity reversal are the only Neveu-Schwarz Verma modules and both are simple. They are called Neveu-Schwarz Fock spaces.

In the Ramond sector, via $\mathfrak{b} \mathfrak{c}_{1 / 2}^{\geq}=\mathfrak{b c}_{1 / 2}^{0} \oplus \mathfrak{b c}_{1 / 2}^{+}$, one has the Verma module

$$
\mathbb{F}^{\mathrm{R}}=\operatorname{Ind}_{\mathfrak{b} \mathfrak{c}_{1 / 2}^{\geq}}^{\mathfrak{b} \mathfrak{c}_{1 / 2}} \mathbb{C}|\mathrm{R}\rangle
$$

where $\mathbb{C}|R\rangle$ is the 1 -dimensional $\mathfrak{b c}_{1 / 2}^{\geq}$module characterised by $|R\rangle$ having even parity and

$$
\mathbf{1}|\mathrm{R}\rangle=|\mathrm{R}\rangle, \quad \mathfrak{b c}_{1 / 2}^{+}|\mathrm{R}\rangle=0 .
$$

This Verma module and its parity reversal are simple and they are the only Ramond Verma modules.

Definition 11. The bc ghost vertex superalgebra bc is the unique vertex superalgebra that is strongly generated by two odd fields $b(z), c(z)$, has the defining operator product expansions

$$
b(z) c(w) \sim-c(w) b(z) \sim \frac{1}{z-w}
$$

and satisfies no additional relations beyond those required by the vertex superalgebra axioms.

As a $\mathfrak{b c}_{0}$ module bc is isomorphic to $\mathbb{F}^{\mathrm{NS}}$.

\section{Free field realisations of the universal vertex operator superalgebra $\mathbf{V}_{\boldsymbol{k}}$}

In this section we consider two free field realisations of $\mathrm{V}_{k}$, once as a subalgebra of $F=H_{1} \otimes G \otimes b c$ and once as a subalgebra of $B=H_{1} \otimes L_{\langle\theta+\psi\rangle} \otimes b c$. 
Proposition 16. Let $\xi \in \mathbb{C} \backslash\{0\}$ and $k=\frac{\xi^{2}-3}{2}$. There exists a vertex operator superalgebra homomorphism $\mathrm{V}_{k} \rightarrow \mathrm{F}$ uniquely characterised by the assignment

$$
\begin{aligned}
& e(z) \mapsto \beta(z) \\
& x(z) \mapsto b(z)+: \beta(z) c(z): \mapsto \xi a(z)+: b(z) c(z):+2: \beta(z) \gamma(z): \\
& h(z) \mapsto\left(\xi^{2}-2\right) \partial c(z)+\xi: a(z) c(c):+: \gamma(z) b(z):+: \beta(z) \gamma(z) c(z):, \\
& y(z) \mapsto(z) \gamma(z) \gamma(z):-\xi^{2}(z) b(z) c(z): \\
& f(z) \mapsto-\xi: a(z) \gamma(z):-: \beta(z) \\
&+\frac{1-\xi^{2}}{2}: \partial c(z) c(z):+\frac{3-\xi^{2}}{2} \partial \gamma(z) .
\end{aligned}
$$

We omit the tensor product symbols for brevity, identifying a with $a \otimes \mathbf{1} \otimes \mathbf{1}$ and so on. The image of the Virasoro field under the above homomorphism is

$$
T(z) \mapsto \frac{1}{2}: a(z) a(z):-\frac{1}{2 \xi} \partial a(z)-: \beta(z) \partial \gamma(z):-\quad: b(z) \partial c(z):
$$

Proof. This free field realisation appears to have first been considered by Bershadsky and Ooguri in [49]. The validity of the proposition follows by checking, through direct calculation, that the generating fields of $\mathrm{V}_{k}$ and their images in $\mathrm{F}$ satisfy the same operator product expansions and that the formulae for the Virasoro fields match. Therefore the above map is a well defined vertex operator superalgebra homomorphism.

Remark 3. The admissible levels of Theorem 5 at which $\mathrm{V}_{k}$ is not simple are realised by the free field realisation whenever $\xi^{2}=u / v$, where $u, v$ are the integer parameters of Theorem 5 .

Since the above homomorphism is non-trivial, it follows that it is injective for the levels at which $\mathrm{V}_{k}$ is simple. To show that the homomorphism remains injective at the levels for which $V_{k}$ is not simple, we need to take a closer look at $F$ modules, which are automatically also $\mathrm{V}_{k}$ modules due to the homomorphism $\mathrm{V}_{k} \rightarrow \mathrm{F}$. Let $|p ; \mathrm{NS}\rangle=|p\rangle \otimes \Omega \otimes|\mathrm{NS}\rangle,|p ; j ; \mathrm{NS}\rangle=|p\rangle \otimes u_{j} \otimes|\mathrm{NS}\rangle,|p ; \mathrm{R}\rangle=|p\rangle \otimes \Omega \otimes|\mathrm{R}\rangle$ and $|p ; j ; \mathrm{R}\rangle=|p\rangle \otimes u_{j} \otimes|\mathrm{R}\rangle$ respectively be relaxed highest weight vectors in

$$
\begin{aligned}
\mathrm{F}_{\mathbb{F}_{p}^{N S}}=\mathbb{F}_{p} \otimes \mathcal{G}^{+} \otimes \mathbb{F}^{\mathrm{NS}}, & \mathrm{F}_{\mathbb{F}_{p ;[j]}^{\mathrm{NS}}}=\mathbb{F}_{p} \otimes \mathcal{W}_{[j]} \otimes \mathbb{F}^{\mathrm{NS}}, \\
\mathrm{F}_{\mathbb{F}_{p}^{\mathrm{R}}}^{\mathrm{R}}=\mathbb{F}_{p} \otimes \mathcal{G}^{+} \otimes \mathbb{F}^{\mathrm{R}} & \mathrm{F}_{\mathbb{F}_{p ;[j]}^{\mathrm{R}}}=\mathbb{F}_{p} \otimes \mathcal{W}_{[j]} \otimes \mathbb{F}^{\mathrm{R}}
\end{aligned}
$$

where $\Omega$ is the highest weight vector of $\mathcal{G}^{+}$and $u_{j}$ a relaxed highest weight vector of $\mathcal{W}_{[j]}$ of weight $j$. Let

$$
\begin{aligned}
\lambda_{p}^{\mathrm{NS}}=\xi p, \quad \lambda_{p ; j}^{\mathrm{NS}} & =\xi p+2 j, \quad \lambda_{p}^{\mathrm{R}}=\xi p-\frac{1}{2}, \quad \lambda_{p ; j}^{\mathrm{R}}=\xi p-\frac{1}{2}+2 j, \\
s_{p} & =\xi p+\frac{1}{2}, \quad q_{p}=\frac{(\xi p+1 / 2)^{2}-1}{2} .
\end{aligned}
$$


From the vertex operator superalgebra homomorphism (20) it then follows that

$$
\begin{aligned}
h_{0}|p ; \mathrm{NS}\rangle & =\lambda_{p}^{\mathrm{NS}}|p ; \mathrm{NS}\rangle, & \Sigma|p ; \mathrm{NS}\rangle & =s_{p}|p ; \mathrm{NS}\rangle, \\
h_{0} c_{0}|p ; \mathrm{NS}\rangle & =\left(\lambda_{p}^{\mathrm{NS}}-1\right) c_{0}|p ; \mathrm{NS}\rangle, & \Sigma c_{0}|p ; \mathrm{NS}\rangle, & =-s_{p} c_{0}|p ; \mathrm{NS}\rangle, \\
h_{0}|p ; j ; \mathrm{NS}\rangle & =\lambda_{p ; j}^{\mathrm{NS}}|p ; j ; \mathrm{NS}\rangle, & \Sigma|p ; j ; \mathrm{NS}\rangle & =s_{p}|p ; j ; \mathrm{NS}\rangle, \\
h_{0} c_{0}|p ; j ; \mathrm{NS}\rangle & =\left(\lambda_{p ; j}^{\mathrm{NS}}-1\right) c_{0}|p ; j ; \mathrm{NS}\rangle, & \Sigma c_{0}|p ; j ; \mathrm{NS}\rangle & =-s_{p} c_{0}|p ; j ; \mathrm{NS}\rangle, \\
h_{0}|p ; \mathrm{R}\rangle & =\lambda_{p}^{\mathrm{R}}|p ; \mathrm{R}\rangle, & Q|p ; \mathrm{R}\rangle & =q_{p}|p ; \mathrm{R}\rangle, \\
h_{0}|p ; j ; \mathrm{R}\rangle & =\lambda_{p ; j}^{\mathrm{R}}|p ; j ; \mathrm{R}\rangle, & Q|p ; j ; \mathrm{R}\rangle & =q_{p}|p ; j ; \mathrm{R}\rangle,
\end{aligned}
$$

where $\Sigma=x_{0} y_{0}-y_{0} x_{0}+1 / 2$ and $Q=h_{0}^{2} / 2+e_{0} f_{0}+f_{0} e_{0}$. Note that while the eigenvalue of $h_{0}$ depends on the $\beta \gamma$ weight of the relaxed highest vector, the eigenvalues of $\Sigma$ and $Q$ do not.

\section{Lemma 17.}

(1) The vertex operator superalgebra homomorphism of Theorem 16 is injective for all $k \in \mathbb{C} \backslash\{-3 / 2\}$ and therefore any singular vectors of $\mathrm{V}_{k}$ will have non-trivial image.

(2) For admissible levels $k=(u-3 v) / 2 v$, the singular vector at $\mathfrak{o s p}(1 \mid 2)$ weight $(u-1) \alpha$ and conformal weight $(u-1) v / 2$ is unique, up to rescaling, in $\mathrm{F}$, where $\mathrm{F}$ is regarded as a $\mathrm{V}_{k}$ module.

It was shown in [46] that $\widehat{\mathfrak{o s p}}(1 \mid 2)$ Verma modules have the same submodule structure as the Virasoro algebra, $\widehat{\mathfrak{s l}}(2)$ and the $N=1$ superconformal algebra. Additionally, since the latter three algebras all share the same submodule structure when decomposing their free field modules (the analogues of Feigin-Fuchs modules for the Virasoro algebra), it would come as no surprise if the $\widehat{\mathfrak{o s p}}(1 \mid 2)$ free field modules also shared this structure. If this were true, then that would imply that singular vectors are unique if they exist, that is, any given weight space contains at most one singular vector up to rescaling. Part 2 of the above lemma would then immediately follow from part 1 . The author is not aware of any literature giving a rigorous decomposition of $\widehat{\mathfrak{o s p}}(1 \mid 2)$ free field modules. Fortunately, detailed knowledge of the decomposition of $\widehat{\mathfrak{o s p}}(1 \mid 2)$ free field modules is not needed here. All that is required is that there is an embedding of $\mathrm{V}_{k}$ and the uniqueness of the singular vector.

Proof. Recall from Theorem 5 that $\mathrm{V}_{k}$ admits at most one singular vector other than the vacuum vector and that this non-trivial singular vector exists if and only if $\mathrm{V}_{k}$ has a proper non-trivial ideal. At the levels $k$ where $\mathrm{V}_{k}$ has no ideals and is thus simple, part 1 follows automatically because the homomorphism is non-zero. To show part 1 at the levels where $\mathrm{V}_{k}$ admits non-trivial proper ideals, we note that the uniqueness of the ideal implies that the image of the homomorphism is isomorphic to either $\mathrm{V}_{k}$ or the affine minimal model vertex operator superalgebra of the same level. Consider the formulae for the $h_{0}$ eigenvalues of $|p ; \mathrm{NS}\rangle$. It is clear that $|p ; \mathrm{NS}\rangle$ is an $\widehat{\mathfrak{o s p}}(1 \mid 2)_{0}$ highest weight vector and as one varies $p$, there are no restrictions on the $\mathfrak{o s p}(1 \mid 2)$ weights that can be obtained. Thus any simple $\mathrm{V}_{k}$ highest weight module can be realised as a subquotient of $\mathrm{F}_{\mathbb{F}_{p}}^{\mathrm{NS}}$ for suitable values 
of $p \in \mathbb{C}$. However, for the affine minimal model vertex operator superalgebras $\mathrm{B}_{0 \mid 1}(u, v)$ not all $\mathfrak{o s p}(1 \mid 2)$ weights are allowed as highest weights. For example, $(u-1) \alpha$, the weight of the singular vector, is not allowed. Thus, the image of the homomorphism must be isomorphic to $\mathrm{V}_{k}$ and not just a quotient thereof.

We show part 2 by contradiction. In order to do so we prepare some results from [46] on the submodule structure and singular vectors of $\widehat{\mathfrak{o s p}}(1 \mid 2)_{0}$ Verma modules. We denote by $\mathcal{V}_{\lambda}$ the Verma at admissible level $k_{u, v}$ generated by a highest weight vector of $\mathfrak{o s p}(1 \mid 2)$ weight $\lambda \alpha$ and by $\mathcal{L}_{\lambda}$ its simple quotient by its unique maximal proper submodule. Specialising [46, Thm. 3.1] to the case at hand shows that the last few terms of the BGG resolution of $\mathcal{L}_{0}, \mathcal{L}_{-1}$ and $\mathcal{L}_{u-1}$ are given by

$$
\begin{aligned}
\cdots \rightarrow & \mathcal{V}_{-u-1} \oplus \mathcal{V}_{2 u-1} \rightarrow \mathcal{V}_{-u} \oplus \mathcal{V}_{u} \rightarrow \mathcal{V}_{-1} \oplus \mathcal{V}_{u-1} \rightarrow \mathcal{V}_{0} \rightarrow \mathcal{L}_{0} \rightarrow 0 \\
& \cdots \rightarrow \mathcal{V}_{-u-1} \oplus \mathcal{V}_{2 u-1} \rightarrow \mathcal{V}_{-u} \oplus \mathcal{V}_{u} \rightarrow \mathcal{V}_{-1} \rightarrow \mathcal{L}_{-1} \rightarrow 0 \\
& \cdots \rightarrow \mathcal{V}_{-u-1} \oplus \mathcal{V}_{2 u-1} \rightarrow \mathcal{V}_{-u} \oplus \mathcal{V}_{u} \rightarrow \mathcal{V}_{u-1} \rightarrow \mathcal{L}_{u-1} \rightarrow 0
\end{aligned}
$$

These resolutions imply that $\mathcal{V}_{0}$ has two independent singular vectors ${ }^{2}$ whose $\mathfrak{o s p}(1 \mid 2)$ and conformal weights are $(-\alpha, 0)$ and $((u-1) \alpha,(u-1) v / 2)$, respectively. These two singular vectors each generate a Verma submodule, and both of these submodules share a pair of independent singular vectors whose $\mathfrak{o s p}(1 \mid 2)$ and conformal weights are $(-u \alpha,(u-1) v / 2)$ and $(u \alpha,(u+1) v / 2)$, respectively. These two additional singular vectors each generate a Verma submodule, and again both of these submodules share a pair of independent singular vectors whose $\mathfrak{o s p}(1 \mid 2)$ and conformal weights are $(-(u+1) \alpha,(u+1) v / 2)$ and $((2 u-1) \alpha,(u+1) v / 2)$, respectively, and so on. As an $\widehat{\mathfrak{o s p}}(1 \mid 2)$ module $\mathrm{V}_{k_{u, v}}$ is isomorphic to $\mathcal{V}_{0} / \mathcal{V}_{-1}$ and, as stated in Theorem 5, this quotient has a unique singular vector at $\mathfrak{o s p}(1 \mid 2)$ and conformal weight $((u-1) \alpha,(u-1) v / 2)$. In part 1 , we showed that $\mathrm{V}_{k_{u, v}}$ is isomorphic to the $\widehat{\mathfrak{o s p}}(1 \mid 2)$ submodule of $\mathrm{F}$ generated by the vector $|0 ; \mathrm{NS}\rangle$. Thus, $\mathrm{U}(\widehat{\mathfrak{o s p}}(1 \mid 2))|0 ; \mathrm{NS}\rangle \cong \mathcal{V}_{0} / \mathcal{V}_{-1}$. A direct calculation shows that $x_{0} c_{0}|0 ; \mathrm{NS}\rangle=|0 ; \mathrm{NS}\rangle$ and therefore, $\mathrm{U}(\widehat{\mathfrak{o s p}}(1 \mid 2)) c_{0}|0 ; \mathrm{NS}\rangle / \mathrm{U}(\widehat{\mathfrak{o s p}}(1 \mid 2))|0 ; \mathrm{NS}\rangle$ is an $\widehat{\mathfrak{o s p}}(1 \mid 2)$ highest weight module of weight $-\alpha$ and is therefore isomorphic to a quotient of $\mathcal{V}_{-1}$.

Due to the weights at which the singular vectors of $\mathcal{V}_{-1}$ appear, the $((u-$ 1) $\alpha,(u-1) v / 2)$ weight spaces of $\mathcal{V}_{-1}$ and $\mathcal{L}_{-1}$ have the same dimension. This implies that neither $\mathcal{V}_{-1}$ nor any quotient of $\mathcal{V}_{-1}$ contains a singular vector in the $((u-1) \alpha,(u-1) v / 2)$ weight space. Further, since both $\mathrm{F}$ and $\mathcal{V}_{0}$ have the same characters as $\widehat{\mathfrak{o s p}}(1 \mid 2)$ modules, their weight spaces also have the same dimensions. Thus, the weight spaces of $F$ have the same dimensions as those of the direct sum $\left(\mathcal{V}_{0} / \mathcal{V}_{-1}\right) \oplus \mathcal{V}_{-1}$. Finally, this implies that the dimensions of the $((u-1) \alpha,(u-1) v / 2)$ weight spaces of $\mathrm{F}$ and $\mathrm{U}\left(\widehat{\mathfrak{o s p}}(1 \mid 2)_{0}\right) c_{0}|0 ; \mathrm{NS}\rangle$ are equal, since the dimension of the $((u-1) \alpha,(u-1) v / 2)$ weight spaces of $\mathcal{V}_{-1}$ and $\mathcal{L}_{-1}$ are.

Assume that the $\widehat{\mathfrak{o s p}}(1 \mid 2)_{0}$ singular vector of weight $((u-1) \alpha,(u-1) v / 2)$ in $\mathrm{F}$ is not unique, then the quotient module $\mathrm{U}\left(\widehat{\mathfrak{o s p}}(1 \mid 2)_{0}\right) c_{0}|0 ; \mathrm{NS}\rangle / \mathrm{U}\left(\widehat{\mathfrak{o s p}}(1 \mid 2)_{0}\right)|0 ; \mathrm{NS}\rangle$ would have to contain at least one singular vector at this weight, because the weight space of $\mathrm{F}$ of weight $((u-1) \alpha,(u-1) v / 2)$ is contained in $\mathrm{U}\left(\widehat{\mathfrak{o s p}}(1 \mid 2)_{0}\right) c_{0}|0 ; \mathrm{NS}\rangle$

\footnotetext{
${ }^{2}$ Independent here meaning that the singular vector is a descendant of the highest weight vector only and not of other singular vectors.
} 
by the above dimension counting arguments. However, neither $\mathcal{V}_{-1}$ nor any of its quotients contain a singular vector at this weight, so in particular, neither can the quotient module $\mathrm{U}\left(\widehat{\mathfrak{o s p}}(1 \mid 2)_{0}\right) c_{0}|0 ; \mathrm{NS}\rangle / \mathrm{U}\left(\widehat{\mathfrak{o s p}}(1 \mid 2)_{0}\right)|0 ; \mathrm{NS}\rangle$.

The second free field realisation of $\mathrm{V}_{k}$ is constructed by embedding the $\beta \gamma$ vertex algebra of the first realisation into a lattice algebra as in Theorem 14.

\section{Lemma 18.}

(1) The composition of the algebra homomorphisms in Theorems 14 and 16 is injective. Therefore any singular vectors of $\mathrm{V}_{k}$ will have non-trivial image in $\mathrm{B}$.

(2) For admissible levels $k=(u-3 v) / 2 v$, the singular vector at $\mathfrak{o s p}(1 \mid 2)$ weight $(u-1) \alpha$ and conformal weight $(u-1) v / 2$ is unique, up to rescaling, in $\mathrm{B}$.

Proof. Part 1 follows from the fact that the two algebra homomorphisms in Theorems 14 and 16 are both injective and thus so is their composition.

We show part 2 by contradiction. As a module over $F$ the vertex superalgebra $\mathrm{B}$ is isomorphic to $\mathbb{F}_{0} \otimes \sigma \mathcal{W}_{0}^{-} \otimes \mathbb{F}^{\mathrm{NS}}$. Further, since $\sigma \mathcal{W}_{0}^{-}$satisfies the non-split exact sequence

$$
0 \rightarrow \mathcal{G}^{+} \rightarrow \sigma \mathcal{W}_{0}^{-} \rightarrow \sigma \mathcal{G}^{+} \rightarrow 0
$$

B satisfies the non-split exact sequence:

$$
0 \rightarrow \mathrm{F} \rightarrow \mathrm{B} \rightarrow \mathbb{F}_{0} \otimes \sigma \mathcal{G}^{+} \otimes \mathbb{F}^{\mathrm{NS}} \rightarrow 0
$$

Since the singular vector at $\mathfrak{o s p}(1 \mid 2)$ and conformal weights $(u-1) \alpha$ and $(u-1) \frac{v}{2}$ is unique in $F$, it is unique in $B$ if and only if there is no $\widehat{\mathfrak{o s p}}(1 \mid 2)$ singular vector at those weights in $\mathbb{F}_{0} \otimes \sigma \mathcal{G}^{+} \otimes \mathbb{F}^{\mathrm{NS}}$. As shall shortly become apparent, no such singular vector exists because $\mathbb{F}_{0} \otimes \sigma \mathcal{G}^{+} \otimes \mathbb{F}^{\mathrm{NS}}$ admits no $\widehat{\mathfrak{o s p}}(1 \mid 2)$ singular vectors at any weight. A necessary condition for a vector to be singular is that it is annihilated by $f_{1}$. We will show that no non-trivial such vector exists by using the fact that $\gamma_{1}$ acts injectively on $\sigma \mathcal{G}^{+}$(this is a consequence of $\gamma_{0}$ acting injectively on $\mathcal{G}^{+}$) to show that $f_{1}$ acts injectively on $\mathbb{F}_{0} \otimes \sigma \mathcal{G}^{+} \otimes \mathbb{F}^{\mathrm{NS}}$. The expansion of $f_{1}$ in free field generators is

$$
\begin{aligned}
f_{1}= & -\xi a_{0} \gamma_{1}-\left(2 \sum_{m \geq 2} \beta_{-m} \gamma_{m}+2 \sum_{m \geq 0} \gamma_{-m} \beta_{m}+\beta_{-1} \gamma_{1}\right) \gamma_{1} \\
& -: b c:{ }_{0} \gamma_{1}-\frac{3-\xi^{2}}{2} \gamma_{1}+C \\
= & -\left(\xi a_{0}+2: \beta \gamma:_{0}+: b c:{ }_{0}-\beta_{-1} \gamma_{1}\right) \gamma_{1}-\frac{3-\xi^{2}}{2} \gamma_{1}+C,
\end{aligned}
$$

where $C$ denotes all summands not containing $\gamma_{1}$, while $: b c:{ }_{0}$ denotes the zero mode of the normally ordered product $: b(z) c(z):$ and $: \beta \gamma:{ }_{0}$ denotes the zero mode of the normally ordered product : $\beta(z) \gamma(z)$ : . Next, we refine the grading of $\mathbb{F}_{0} \otimes \sigma \mathcal{G}^{+} \otimes \mathbb{F}^{\mathrm{NS}}$ by Heisenberg, $\beta \gamma$ and $b c$ weights to also include the eigenvalue of $\beta_{-1} \gamma_{1}$ (on $\sigma \mathcal{G}^{+}$the eigenvalues of $\beta_{-1} \gamma_{1}$ are all integers). When acting on a homogeneous vector with respect to this refined grading, the terms collected in $C$ in the expansion of $f_{1}$ either do not change the eigenvalue of $\beta_{-1} \gamma_{1}$ or increase 
the eigenvalue by a positive integer if they contain factors of $\beta_{-1}$. Conversely, the summands containing $\gamma_{1}$ shift the eigenvalue of $\beta_{-1} \gamma_{1}$ by -1 . Let $w$ be a homogeneous vector with respect to the refined grading, then

$$
\begin{aligned}
f_{1} w & =\left(\xi a_{0}+2: \beta \gamma:_{0}+: b c:_{0}-\beta_{-1} \gamma_{1}\right) \gamma_{1} w-\frac{3-\xi^{2}}{2} \gamma_{1} w+C w \\
& =\left(m+2-\frac{u+v}{2 v}\right) \gamma_{1} w+C w
\end{aligned}
$$

where $m \in \mathbb{Z}$ is the eigenvalue of $\xi a_{0}+2: \beta \gamma:_{0}+: b c::_{0}-\beta_{-1} \gamma_{1}$ and we have used the fact that $\xi^{2}=u / v$. Since $v$ and $(u+v) / 2$ are coprime, $(u+v) / 2 v$ is not an integer and the coefficient of $\gamma_{1} w$ is therefore non-zero. Further, $\gamma_{1} w$ and $C w$ have different $\beta_{-1} \gamma_{1}$ eigenvalues, so they cannot be linearly dependent. Thus, $f_{1} w \neq 0$ for any homogeneous vector of the refined grading and $f_{1}$ acts injectively.

Remark 4. The importance of the uniqueness of the singular vectors in Theorems 17 and 18 will become apparent in Section 4, where these singular vectors will be constructed as images of highest weight under screening operators. Since screening operators are module homomorphisms it follows immediately that these images are singular vectors, but without uniqueness it would not be clear that the correct singular vectors have been constructed.

The second free field realisation implies that modules over B are also modules over $\mathrm{V}_{k}$ by restriction. We introduce the following notation

$$
\begin{array}{ll}
\mathrm{B}_{\mathbb{F}_{p}^{\mathrm{NS}}}^{\mathrm{NS}}=\mathbb{F}_{p} \otimes \mathbb{F}_{[0]} \otimes \mathbb{F}^{\mathrm{NS}}, & \mathrm{B}_{\mathbb{F}_{p ;[\lambda], n}^{\mathrm{NS}}}^{\mathrm{NS}}=\mathbb{F}_{p} \otimes \mathbb{F}_{[\lambda(\psi+\theta)+n \psi]} \otimes \mathbb{F}^{\mathrm{NS}}, \\
\mathrm{B}_{\mathbb{F}_{p}^{\mathrm{R}}}^{\mathrm{R}}=\mathbb{F}_{p} \otimes \mathbb{F}_{[0]} \otimes \mathbb{F}^{\mathrm{R}}, & \mathrm{B}_{\mathbb{F}_{p ;[\lambda], n}^{\mathrm{R}}}^{\mathrm{R}}=\mathbb{F}_{p} \otimes \mathbb{F}_{[\lambda(\psi+\theta)+n \psi]} \otimes \mathbb{F}^{\mathrm{R}}
\end{array}
$$

We now turn screening operators - the original introduced by Dotsenko and Fateev in the context of the Coulomb gas formalism [20] — as a means of constructing $\mathrm{V}_{k}$ singular vectors.

Definition 12. Let $\mathrm{V}$ be a vertex operator superalgebra together with a free field realisation, $\mathrm{V} \hookrightarrow \mathrm{W}$, in a free field vertex operator superalgebra $\mathrm{W}$. A screening field is a field corresponding to a vector in a module over $\mathbf{W}$, whose operator product expansions with the fields of $\mathrm{V}$ have singular parts that are total derivatives. It suffices to check this for the generating fields of $V$.

Proposition 19. The field

$$
\mathcal{Q}_{1}(z)=(\beta(z) c(z)-b(z)) \mathrm{V}_{-a / \xi}(z)
$$

is a screening field for the free field realisations $\mathrm{V}_{k} \hookrightarrow \mathrm{F}$ and also for $\mathrm{V}_{k} \hookrightarrow \mathrm{B}$ when $\beta(z)$ is realised as $\beta(z)=\mathrm{V}_{\theta+\psi}(z)$. The field

$$
\mathcal{Q}_{2}(z)=(\beta(z) c(z)-b(z)) \mathrm{V}_{\xi a-\left(1+\xi^{2}\right)(\theta+\psi) / 2}(z)
$$

is a screening field for $\mathrm{V}_{k} \hookrightarrow \mathrm{B}$, where again $\beta(z)=\mathrm{V}_{\theta+\psi}(z)$. 
Proof. The proposition follows by direct computation, that is, computing the operator product expansions of the generators of $\mathrm{V}_{k}$ with the screening fields $\mathcal{Q}_{1}(z)$ and $\mathcal{Q}_{2}(z)$ and verifying that they are indeed total derivatives. For $\mathcal{Q}_{1}(z)$ the nonzero operator product expansions are

$$
y(z) \mathcal{Q}_{1}(w) \sim \partial_{w} \frac{\xi^{2} \mathrm{~V}_{-a / \xi}(w)}{z-w}, \quad f(z) \mathcal{Q}_{1}(w) \sim \partial_{w} \frac{\xi^{2} c(w) \mathrm{V}_{-a / \xi}(w)}{z-w}
$$

while for $\mathcal{Q}_{2}(z)$ they are

$$
\begin{aligned}
y(z) & \mathcal{Q}_{2}(w) \\
& \sim \partial_{w} \frac{\mathrm{V}_{\xi a-\left(1+\xi^{2}\right)(\theta+\psi) / 2}(w)}{z-w}, \\
f(z) \mathcal{Q}_{2}(w) & \\
& \sim \partial_{w} \frac{\frac{1+\xi^{2}}{2} b(w) \mathrm{V}_{\xi a-\left(3+\xi^{2}\right)(\theta+\psi) / 2}(w)+\frac{1-\xi^{2}}{2} c(w) \mathrm{V}_{\xi a-\left(1+\xi^{2}\right)(\theta+\psi) / 2}(w)}{z-w} .
\end{aligned}
$$

Remark 5. While screening operators of the form (21) are well known, those of the form (22) are less commonly considered. They have appeared in the physics literature as formal non-integer powers of screening operators, that is, through identities of the form " $\mathcal{Q}_{2}(z)=\mathcal{Q}_{1}(z)^{-\xi^{2}}$ " see for example [50]. In [51] such noninteger powers were interpreted as formal quantities which were to be expanded within normally ordered products in a way that avoids non-integer powers.

Here, instead, we enlarge the $\beta \gamma$ vertex algebra, by embedding it into a lattice vertex algebra, through a free field realisation. In this larger vertex algebra noninteger powers of $\beta(z)=\mathrm{V}_{\theta+\psi}(z)$ can be defined as $\beta(z)^{\lambda}=\mathrm{V}_{\lambda(\theta+\psi)}(z)$ which is well defined because $\theta+\psi$ has norm 0 and thus, for all $\lambda, \mu \in \mathbb{C}$, we have $\mathrm{V}_{\lambda(\theta+\psi)}(z) \mathrm{V}_{\mu(\theta+\psi)}(z)=\mathrm{V}_{(\lambda+\mu)(\theta+\psi)}(z)$.

The interest in screening fields, for a free field realisation $V \hookrightarrow W$, stems from the fact that their residues, when well defined, commute with the action of $\mathrm{V}$. These residues, referred to as screening operators, thus define $\mathrm{V}$ module homomorphisms. This implies that the image of any singular vector will again be singular (or zero). Thus screening fields and operators are an invaluable tool for constructing singular vectors in $\mathrm{V}$ modules.

Consider the composition of $\ell$ copies of the screening field $\mathcal{Q}_{1}(z)$ :

$$
\begin{aligned}
& \mathcal{Q}_{1}\left(z_{1}\right) \cdots \mathcal{Q}_{1}\left(z_{\ell}\right) \\
& =\left(\beta\left(z_{1}\right) c\left(z_{1}\right)-b\left(z_{1}\right)\right) \cdots\left(\beta\left(z_{\ell}\right) c\left(z_{\ell}\right)-b\left(z_{\ell}\right)\right) \mathrm{e}^{-\ell / \xi a} \prod_{1 \leq i<j \leq \ell}\left(z_{i}-z_{j}\right)^{\xi^{-2}} \\
& \quad \cdot \prod_{i=1}^{\ell} z_{i}^{-a_{0} / \xi} \cdot \prod_{m \geq 1}\left[\exp \left(\frac{-1}{\xi} \frac{a_{-m}}{m} \sum_{i=1}^{\ell} z_{i}^{m}\right) \exp \left(\frac{1}{\xi} \frac{a_{m}}{m} \sum_{i=1}^{\ell} z_{i}^{-m}\right)\right]
\end{aligned}
$$

The action of screening fields and operators is most easily evaluated using methods coming from the theory of symmetric functions, however, products of fermions such 
as those above are skew-symmetric. This obstacle can be overcome by factoring out the Vandermonde determinant $\Delta(z)=\prod_{1 \leq i<j \leq \ell}\left(z_{i}-z_{j}\right)$ :

$$
\begin{aligned}
\prod_{1 \leq i<j \leq \ell}\left(z_{i}-z_{j}\right)^{\xi^{-2}} & =\Delta(z) \prod_{1 \leq i<j \leq \ell}\left(z_{i}-z_{j}\right)^{\xi^{-2}-1} \\
& =\Delta(z) \prod_{1 \leq i \neq j \leq \ell}\left(z_{i}-z_{j}\right)^{\left(\xi^{-2}-1\right) / 2}
\end{aligned}
$$

where we have suppressed a complex phase in the second equality which will later be absorbed into integration cycles. This allows us to rewrite the product of screening fields (23) in the form

$$
\begin{aligned}
\mathcal{Q}_{1}\left(z_{1}\right) \cdots & \mathcal{Q}_{1}\left(z_{\ell}\right) \\
= & \mathrm{e}^{-\ell / \xi a} \prod_{1 \leq i \neq j \leq \ell}\left(1-\frac{z_{i}}{z_{j}}\right)^{\left(\xi^{-2}-1\right) / 2} \\
& \cdot \prod_{i=1}^{\ell} z_{i}^{-a_{0} / \xi+(\ell-1)\left(\xi^{-2}-1\right) / 2} \\
& \cdot \Delta(z)\left(\beta\left(z_{1}\right) c\left(z_{1}\right)-b\left(z_{1}\right)\right) \cdots\left(\beta\left(z_{\ell}\right) c\left(z_{\ell}\right)-b\left(z_{\ell}\right)\right) \\
& \cdot \prod_{m \geq 1}\left[\exp \left(\frac{-1}{\xi} \frac{a_{-m}}{m} \sum_{i=1}^{\ell} z_{i}^{m}\right) \exp \left(\frac{1}{\xi} \frac{a_{m}}{m} \sum_{i=1}^{\ell} z_{i}^{-m}\right)\right]
\end{aligned}
$$

where the skew symmetry of the fermions is now countered by that of the Vandermonde determinant.

To define screening operators as integrals of products of screening fields, there need to exist cycles over which to integrate. The obstruction to the existence of such cycles lies in the multivaluedness of the second product on the righthand side of $(24)$. If the exponent $-a_{0} / \xi+(\ell-1)\left(\xi^{-2}-1\right) / 2$ evaluates to an integer, when acting on a Neveu-Schwarz free field module, then there exists such a cycle $\Gamma(\ell, \xi)$, generically unique in homology up to normalisation and constructed in [21]. For example, on the free field module $\mathbb{F}_{p} \otimes \mathcal{W}_{[j]} \otimes \mathbb{F}^{\mathrm{NS}}, a_{0}$ acts as $p$ and the exponent evaluates to $-p / \xi+(\ell-1)\left(\xi^{-2}-1\right) / 2$. The cycles $\Gamma(\ell, \xi)$ are homologically equivalent to the cycles over which one integrates in the theory of symmetric polynomials to define inner products - see [32, Sect. 3] for details. The actual construction of the cycles $\Gamma(\ell, \xi)$ is rather subtle and we refer the interested reader to [21] for the complete picture.

For completeness, we mention that when acting on a Ramond free field module, the cycles $\Gamma(\ell, \xi)$ exist when $-a_{0} / \xi+(\ell-1)\left(\xi^{-2}-1\right) / 2$ evaluates to a half integer (to compensate for the half integer exponents of free fermion fields). Screening operators between Ramond modules shall, however, not be needed in what follows.

Analogously, composing $\ell$ copies of the screening field $\mathcal{Q}_{2}(z)$ yields 


$$
\begin{aligned}
\mathcal{Q}_{2}\left(z_{1}\right) \cdots \mathcal{Q}_{2}\left(z_{\ell}\right) \\
=\mathrm{e}^{\ell \xi a-\ell\left(1+\xi^{2}\right)(\theta+\psi) / 2} \\
\quad \cdot \prod_{1 \leq i \neq j \leq \ell}\left(1-\frac{z_{i}}{z_{j}}\right)^{\left(\xi^{2}-1\right) / 2} \prod_{i=1}^{\ell} z_{i}^{\xi a_{0}+(\ell-1)\left(\xi^{2}-1\right) / 2-\left(1+\xi^{2}\right)\left(\theta_{0}+\psi_{0}\right) / 2} \\
\cdot \Delta(z)\left(\beta\left(z_{1}\right) c\left(z_{1}\right)-b\left(z_{1}\right)\right) \cdots\left(\beta\left(z_{\ell}\right) c\left(z_{\ell}\right)-b\left(z_{\ell}\right)\right) \\
\quad \cdot \prod_{m \geq 1}\left[\exp \left(\xi \frac{a_{-m}}{m} \sum_{i=1}^{\ell} z_{i}^{m}\right) \exp \left(-\xi \frac{a_{m}}{m} \sum_{i=1}^{\ell} z_{i}^{-m}\right)\right] \\
\quad \cdot \prod_{m \geq 1}\left[\exp \left(-\frac{1+\xi^{2}}{2} \frac{\theta_{-m}+\psi_{-m}}{m} \sum_{i=1}^{\ell} z_{i}^{m}\right) \exp \left(\frac{1+\xi^{2}}{2} \frac{\theta_{m}+\psi_{m}}{m} \sum_{i=1}^{\ell} z_{i}^{-m}\right)\right],
\end{aligned}
$$

Definition 13. Let $\ell$ and $m$ be integers, and $\ell \geq 1$.

(1) For $p=(\ell-2 m-1) / 2 \xi-\xi(\ell-1) / 2$, the screening operators $\mathcal{Q}_{1}^{[\ell]}:{ }^{\mathrm{F}_{F}}{ }_{p}^{\mathrm{NS}} \rightarrow$ $\mathrm{F}_{\mathbb{F}_{p-\ell / \xi}^{N S}}$ and $\mathcal{Q}_{1}^{[\ell]}:{ }_{\mathbb{F}^{N}}^{\mathrm{NS}} \rightarrow \mathrm{F}_{\mathbb{F}_{p-\ell / \xi ; j}^{N S}}^{\mathrm{N}}$ are defined by

$$
\mathcal{Q}_{1}^{[\ell]}=\int_{\Gamma(\ell, \xi)} \mathcal{Q}_{1}\left(z_{1}\right) \cdots \mathcal{Q}_{1}\left(z_{\ell}\right) \mathrm{d} z_{1} \cdots \mathrm{d} z_{\ell},
$$

meaning that the cycle $\Gamma(\ell, \xi)$ exists.

(2) For $p=(\ell+2 m-1) / 2 \xi-\xi(\ell-1) / 2$, the screening operators $\mathcal{Q}_{2}^{[\ell]}:{ }^{\mathrm{B}} \mathbb{F}_{p}^{\mathrm{NS}} \rightarrow$

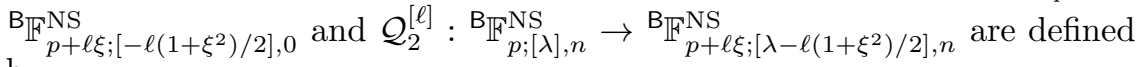
by

$$
\mathcal{Q}_{2}^{[\ell]}=\int_{\Gamma\left(\ell, \xi^{-1}\right)} \mathcal{Q}_{2}\left(z_{1}\right) \cdots \mathcal{Q}_{2}\left(z_{\ell}\right) \mathrm{d} z_{1} \cdots \mathrm{d} z_{\ell},
$$

meaning that the cycle $\Gamma\left(\ell, \xi^{-1}\right)$ exists.

We normalise these cycles such that

$$
\int_{\Gamma\left(\ell, \xi^{ \pm 1}\right)} \prod_{1 \leq i \neq j \leq \ell}\left(1-\frac{z_{i}}{z_{j}}\right)^{\left(\xi^{\mp 2}-1\right) / 2} \frac{\mathrm{d} z_{1} \cdots \mathrm{d} z_{\ell}}{z_{1} \cdots z_{\ell}}=1 .
$$

We shall lighten notation in what follows by suppressing the cycle $\Gamma\left(\ell, \xi^{ \pm 1}\right)$ in all integrals.

Remark 6. As mentioned previously, the

$$
\Delta(z)\left(\beta\left(z_{1}\right) c\left(z_{1}\right)-b\left(z_{1}\right)\right) \cdots\left(\beta\left(z_{\ell}\right) c\left(z_{\ell}\right)-b\left(z_{\ell}\right)\right)
$$

factor and the factors of exponential functions that appear on the right-hand side of (24) and (25) are both invariant under permuting the $z_{i}$. The action of the screening operators $\mathcal{Q}_{1}^{[\ell]}$ and $\mathcal{Q}_{1}^{[\ell]}$ can thus be evaluated using the well-studied family of inner products of symmetric polynomials defined by 


$$
\langle f, g\rangle_{\ell}^{t}=\int \prod_{1 \leq i \neq j \leq \ell}\left(1-\frac{z_{i}}{z_{j}}\right)^{1 / t} f\left(z_{1}^{-1}, \ldots, z_{\ell}^{-1}\right) g\left(z_{1}, \ldots, z_{\ell}\right) \frac{\mathrm{d} z_{1} \cdots \mathrm{d} z_{\ell}}{z_{1} \cdots z_{\ell}}
$$

where $f$ and $g$ are symmetric polynomials and $t \in \mathbb{C} \backslash\{0\}$. One of the characterising properties of Jack symmetric polynomials $\mathrm{P}_{\lambda}^{t}(z)$ is that they are orthogonal with respect to these inner products labelled by $t$. For readers unfamiliar with Jack symmetric polynomials we recommend Macdonald's comprehensive book [52], while for readers familiar with Jack symmetric polynomials, a short summary of their properties and the notation used here can be found in [25, App. A].

\section{Free field correlation functions}

In this section we derive identities involving the correlation functions of the Heisenberg, $\beta \gamma$ and $b c$ fields. These will be needed for evaluating the action of the zero modes of singular vectors in Section 4.

Let $\mathbb{F}_{p}^{*}$ be the graded dual of the highest-weight $\mathrm{H}_{r}$-module $\mathbb{F}_{p}, p \in \mathcal{H}_{r}$. Then, $\mathbb{F}_{p}^{*}$ is a lowest-weight right $\mathfrak{H}_{r}$-module generated by a lowest-weight vector $\langle p|$ satisfying

$$
\langle p \mid p\rangle=1, \quad\langle p| \mathfrak{H}_{r}^{-}=0 .
$$

It is convenient to extend the domain of the functionals in $\mathbb{F}_{p}^{*}$ to all Fock spaces $\mathbb{F}_{q}, q \in \mathcal{H}_{r}$, but to have them act trivially unless $q=p$.

Definition 14. Let $B$ be any combination of normally ordered products of free bosons $a(z)$, vertex operators $\mathrm{V}_{p}(z)$ and their derivatives. The free boson correlation function in $\mathbb{F}_{p}$ and $\mathbb{F}_{q}^{*}$ is then defined to be $\langle q|B| p\rangle$.

The operator product expansion formulae (14) then imply the following wellknown correlation function formulae.

Proposition 20. The correlation function of $k$ vertex operators is given by

$$
\left\langle q\left|\mathrm{~V}_{p_{1}}\left(z_{1}\right) \cdots \mathrm{V}_{p_{k}}\left(z_{k}\right)\right| p\right\rangle=\delta_{q, p_{1}+\cdots+p_{k}+p} \prod_{1 \leq i<j \leq k}\left(z_{i}-z_{j}\right)^{\left(p_{i}, p_{j}\right)} \cdot \prod_{i=1}^{k} z_{i}^{\left(p, p_{i}\right)}
$$

Let $\mathcal{G}^{+*}$ be the graded dual of the highest weight $\mathfrak{G}_{\beta \gamma}$ module $\mathcal{G}^{+}$. Then, $\mathcal{G}^{+*}$ is a lowest-weight right $\mathfrak{G}_{\beta \gamma}$ module generated by a lowest-weight vector $\phi$ satisfying

$$
\langle\phi \mid \Omega\rangle=1, \quad \phi \mathfrak{G}_{\beta \gamma}^{-}=0 .
$$

Further, let $\mathcal{W}_{q}^{*}$ be the graded dual of the highest weight $\mathfrak{G}_{\beta \gamma}$ module $\mathcal{W}_{q}$. Then, $\mathcal{W}_{q}^{*}$ is a lowest-weight right $\mathfrak{G}_{\beta \gamma}$ module generated by a lowest-weight vector $\phi_{q}$ satisfying

$$
\left\langle\phi_{q} \mid u_{q}\right\rangle=1, \quad \phi_{q} \mathfrak{G}_{\beta \gamma}^{-}=0 .
$$

Definition 15. Let $B$ be any combination of normally ordered products of $\mathrm{G}$ fields. The bosonic ghost correlation functions in $\mathcal{G}^{+}$and $\mathcal{W}_{q}$ are then respectively defined to be

$$
\langle B\rangle=\langle\phi|B| \Omega\rangle, \quad\langle B\rangle^{q}=\left\langle\phi_{q}|B| u_{q}\right\rangle
$$


Lemma 21. Let $n$ be a non-negative integer, $q \in \mathbb{C}$ and let $(q)_{n}$ be the rising factorial $\prod_{i=1}^{n}(q+i-1)$. The $\beta, \gamma$ fields satisfy

$$
\begin{aligned}
\left\langle\gamma_{1}^{n} \beta\left(z_{1}\right) \cdots \beta\left(z_{n}\right)\right\rangle & =n ! \\
\left\langle\gamma_{0}^{n} \beta\left(z_{1}\right) \cdots \beta\left(z_{n}\right)\right\rangle^{q} & =(q)_{n} \prod_{i=1}^{n} z_{i}^{-1} .
\end{aligned}
$$

Proof. The formulae follow by induction after noting that $\left[\gamma_{n}, \beta(z)\right]=\mathbf{1} z^{-n}$, that $\gamma_{1} \Omega=0$ and that $\gamma_{0} \beta_{0} u_{q}=q u_{q}$.

Let $\mathbb{F}^{\mathrm{NS} *}$ be the graded dual of the highest weight $\mathfrak{b} \mathfrak{c}_{0}$ module $\mathbb{F}^{\mathrm{NS}}$. Then, $\mathbb{F}^{\mathrm{NS} *}$ is a lowest-weight right $\mathfrak{b} \mathfrak{c}_{0}$ module generated by a lowest-weight vector $\langle\mathrm{NS}|$ satisfying

$$
\langle\mathrm{NS} \mid \mathrm{NS}\rangle=1, \quad\left\langle\mathrm{NS}\left|b_{0} c_{0}\right| \mathrm{NS}\right\rangle=1, \quad\langle\mathrm{NS}| \mathfrak{b c}_{0}^{-}=0 .
$$

Further, let $\mathbb{F}^{\mathrm{R} *}$ be the graded dual of the highest weight $\mathfrak{b c}_{1 / 2}$ module $\mathbb{F}^{\mathrm{R}}$. Then, $\mathbb{F}^{\mathrm{R} *}$ is a lowest-weight right $\mathfrak{b c}_{1 / 2}$ module generated by a lowest-weight vector $\langle\mathrm{R}|$ satisfying

$$
\langle\mathrm{R} \mid \mathrm{R}\rangle=1, \quad\langle\mathrm{R}| \mathfrak{b} \mathfrak{c}_{1 / 2}^{-}=0 .
$$

Definition 16. Let $B$ be any combination of normally ordered products of bc fields. The fermionic ghost correlation functions in $\mathbb{F}^{\mathrm{NS}}$ and $\mathbb{F}^{\mathrm{R}}$ are then defined to be

$$
\langle B\rangle_{\mathrm{NS}}^{+}=\langle\mathrm{NS}|B| \mathrm{NS}\rangle, \quad\langle B\rangle_{\mathrm{NS}}^{-}=\left\langle\mathrm{NS}\left|b_{0} B c_{0}\right| \mathrm{NS}\right\rangle, \quad\langle B\rangle_{\mathrm{R}}=\langle\mathrm{R}|B| \mathrm{R}\rangle .
$$

Correlation functions involving fermions can often be efficiently expressed using pfaffians and this remains true for $b c$ ghosts. The determinant of a skew-symmetric matrix $A=-A^{\top}$ can always be written as the square of a polynomials in the coefficients of $A$. This polynomial, up to a choice of sign, is the pfaffian $\operatorname{pf}(A)$ of $A$.

Definition 17. Let $A$ be a $2 n \times 2 n$ skew-symmetric matrix, so that $A$ is uniquely determined by its upper-triangular entries $A_{i, j}, i<j$. We shall write $A=$ $\left(A_{i, j}\right)_{1 \leq i<j \leq 2 n}$ to indicate a skew-symmetric matrix $A$ with given upper-triangular entries. After defining the pfaffian of the $0 \times 0$ matrix to be 1 , the pfaffian of $A$ can be recursively defined by

$$
\operatorname{pf}(A)=\sum_{\substack{j=1 \\ j \neq i}}^{2 n}(-1)^{i+j+\theta(j-i)} A_{i, j} \operatorname{pf}\left(A_{\hat{\imath}, \hat{\jmath}}\right),
$$

where the row index $i$ may be chosen arbitrarily, $A_{\hat{\imath}, \hat{\jmath}}$ denotes the matrix $A$ with the $i$ th and $j$ th rows and columns removed, and

$$
\theta(x)= \begin{cases}1 & \text { if } x>0 \\ 0 & \text { if } x<0\end{cases}
$$

is the Heaviside step function. In particular, $i=1$ gives the simplified formula

$$
\operatorname{pf}(A)=\sum_{j=2}^{2 n}(-1)^{j} A_{1, j} \operatorname{pf}\left(A_{\hat{1}, \hat{\jmath}}\right) .
$$


Lemma 22. Let $n$ be a non-negative integer and $m \in \mathbb{Z}$. The $\beta \gamma$ and bc fields satisfy the following correlation function formulae.

(1) In the Neveu-Schwarz sector,

$$
\begin{aligned}
& \left\langle\prod_{i=1}^{2 n}\left(c\left(z_{i}\right) z_{i}^{m}-b\left(z_{i}\right)\right)\right\rangle_{\mathrm{NS}}^{+}=(-1)^{n} \operatorname{pf}\left(\frac{z_{i}^{m}+z_{j}^{m}}{z_{i}-z_{j}}\right)_{1 \leq i<j \leq 2 n}, \\
& \left\langle\prod_{i=1}^{2 n}\left(c\left(z_{i}\right) z_{i}^{m}-b\left(z_{i}\right)\right)\right\rangle_{\mathrm{NS}}^{-}=(-1)^{n} \operatorname{pf}\left(\frac{z_{i}^{m+1} z_{j}^{-1}+z_{i}^{-1} z_{j}^{m+1}}{z_{i}-z_{j}}\right)_{1 \leq i<j \leq 2 n} .
\end{aligned}
$$

(2) In the Ramond sector,

$$
\left\langle\prod_{i=1}^{2 n}\left(c\left(z_{i}\right) z_{i}^{m}-b\left(z_{i}\right)\right)\right\rangle_{\mathrm{R}}=(-1)^{n} \mathrm{pf}\left(\frac{z_{i}^{m+\frac{1}{2}} z_{j}^{-\frac{1}{2}}+z_{i}^{-\frac{1}{2}} z_{j}^{m+\frac{1}{2}}}{z_{i}-z_{j}}\right)_{1 \leq i<j \leq 2 n} .
$$

Proof. The above identities all follow by induction using the recursive definition of pfaffians. We give the details for the first identity and leave the rest as an exercise for the reader.

The base step of the induction follows from the fact that for $n=0$ the correlator is empty and therefore equal to 1 . For the inductive step, assume the identity is true for $n-1$ and consider

$$
\begin{aligned}
& \left\langle\prod_{i=1}^{2 n}\left(c\left(z_{i}\right) z_{i}^{m}-b\left(z_{i}\right)\right)\right\rangle_{\mathrm{NS}}^{+}=\left\langle\left(c\left(z_{1}\right) z_{1}^{m}-b\left(z_{1}\right)\right) \prod_{i=2}^{2 n}\left(c\left(z_{i}\right) z_{i}^{m}-b\left(z_{i}\right)\right)\right\rangle_{\mathrm{NS}}^{+} \\
& =\sum_{k \geq 1} z_{1}^{m-k}\left\langle c_{k} \prod_{i=2}^{2 n}\left(c\left(z_{i}\right) z_{i}^{m}-b\left(z_{i}\right)\right)\right\rangle_{\mathrm{NS}}^{+}-\sum_{k \geq 0} z_{1}^{-k-1}\left\langle b_{k} \prod_{i=2}^{2 n}\left(c\left(z_{i}\right) z_{i}^{m}-b\left(z_{i}\right)\right)\right\rangle_{\mathrm{NS}}^{+} \\
& =\sum_{j=2}^{2 n}(-1)^{j-1}\left\langle\prod_{\substack{i=2 \\
i \neq j}}^{2 n}\left(c\left(z_{i}\right) z_{i}^{-1}-b\left(z_{i}\right)\right)\right\rangle_{\mathrm{NS}}^{+} \sum_{k \geq 0}\left(z_{1}^{m-k-1} z_{j}^{k}+z_{1}^{-k-1} z_{j}^{k+m}\right) \\
& =\sum_{j=2}^{2 n}(-1)^{j}\left(-1 \frac{z_{1}^{m}+z_{j}^{m}}{z_{1}-z_{j}}\right)\left\langle\prod_{\substack{i=2 \\
i \neq j}}^{2 n}\left(c\left(z_{i}\right) z_{i}^{-1}-b\left(z_{i}\right)\right)\right\rangle_{\mathrm{NS}}^{+}=\operatorname{pf}\left(-\frac{z_{i}^{m}+z_{j}^{m}}{z_{i}-z_{j}}\right)_{1 \leq i<j \leq 2 n} \\
& =(-1)^{n} \operatorname{pf}\left(\frac{z_{i}^{m}+z_{j}^{m}}{z_{i}-z_{j}}\right)_{1 \leq i<j \leq 2 n}
\end{aligned}
$$

where the third equality follows from the anti-commutation relations $\left\{c_{k}, b(z)\right\}=$ $\mathbf{1} z^{k-1}$ and $\left\{b_{k}, c(z)\right\}=\mathbf{1} z^{k}$ and the fifth from the recursive definition of pfaffians.

The above correlation functions involve fermions and so it is unsurprising that they are skew-symmetric with respect to permuting the $z_{i}$ variables. This skewsymmetry can be compensated for by multiplying the above correlation functions 
with the Vandermonde determinant. In addition to being symmetric, the products of the above correlation functions with the Vandermonde determinant also vanish whenever 3 variables coincide. Symmetric polynomials with this property form an ideal of the ring of symmetric polynomials. This ideal and generalisations thereof were studied by by Feigin and Jimbo and Miwa and Mukhin [31]. This ideal is spanned by Jack symmetric functions at parameter value $t=-3$ whose associated partitions satisfy certain admissibility conditions. We refer the reader to [34, Sect. 3] for a detailed account of how to apply Feigin and Jimbo and Miwa and Mukhin's work to correlation functions and for the definitions of the notation used below. See in particular the notation introduced in Definitions 3.1 and 3.4 for the admissible partitions $\delta^{(m)}\left(n_{1}, n_{2}\right)$ and $\epsilon^{(m)}(n)$, and the involution of partitions $\left[k-\epsilon^{(m)}(n)\right]$ in Lemma 3.5 of [34], as they shall be used repeatedly in what follows.

Let $B$ be some combination of $\beta \gamma$ and $b c$ fields, then we denote the combined correlation functions by $\langle B\rangle_{\mathrm{NS}}^{q ; \pm}=\left\langle\langle B\rangle^{q}\right\rangle_{\mathrm{NS}}^{ \pm},\langle B\rangle_{\mathrm{R}}^{q}=\left\langle\langle B\rangle^{q}\right\rangle_{\mathrm{R}}$ and we abbreviate $\langle\langle B\rangle\rangle_{\mathrm{NS}}^{ \pm}$as $\langle B\rangle_{\mathrm{NS}}^{ \pm}$.

Lemma 23. Let $n$ be a non-negative integer and $q \in \mathbb{C}$.

(1) In the Neveu-Schwarz sector,

$$
\begin{aligned}
& \prod_{i=1}^{2 n} z_{i}^{-2 n+2} \Delta(z)\left\langle\gamma_{1}^{n} \prod_{i=1}^{2 n}\left(\beta\left(z_{i}\right) c\left(z_{i}\right)-b\left(z_{i}\right)\right)\right\rangle_{\mathrm{NS}}^{+} \\
& \quad=n ! \prod_{i=1}^{2 n} z_{i}^{-2 n+2} \Delta(z)\left\langle\prod_{i=1}^{2 n}\left(c\left(z_{i}\right)-b\left(z_{i}\right)\right)\right\rangle_{\mathrm{NS}}^{+}=n !(-2)^{n} \mathrm{P}_{\delta^{(2 n)}(0,0)}^{-3}\left(z^{-1}\right) \\
& \prod_{i=1}^{2 n+1} z_{i}^{-2 n} \Delta(z)\left\langle\gamma_{1}^{n} b_{0} \prod_{i=1}^{2 n+1}\left(\beta\left(z_{i}\right) c\left(z_{i}\right)-b\left(z_{i}\right)\right)\right\rangle_{\mathrm{NS}}^{+} \\
& \quad=n ! \prod_{i=1}^{2 n+1} z_{i}^{-2 n} \Delta(z)\left\langle b_{0} \prod_{i=1}^{2 n+1}\left(c\left(z_{i}\right)-b\left(z_{i}\right)\right)\right\rangle_{\mathrm{NS}}^{+}-n !(-2)^{n+1} \mathrm{P}_{\delta^{(2 n)}(2,0)}^{-3}\left(z^{-1}\right)
\end{aligned}
$$

and

$$
\begin{aligned}
& \prod_{i=1}^{2 n} z_{i}^{-2 n+1} \Delta(z)\left\langle\gamma_{0}^{n} \prod_{i=1}^{2 n}\left(\beta\left(z_{i}+w\right) c\left(z_{i}+w\right)-b\left(z_{i}+w\right)\right)\right\rangle_{\mathrm{NS}}^{q ; \pm} \\
& \quad=(q)_{n}(-1)^{n} \prod_{i=1}^{2 n}\left(z_{i}+w\right)^{-1} \sum_{i=0}^{n} w^{n-i} c_{i}^{(2 n)} \mathrm{P}_{\left[2 n-1-\epsilon^{(2 n)}(i)\right]}^{-3}\left(z^{-1}\right) \\
& \prod_{i=1}^{2 n+1} z_{i}^{-2 n-1} \Delta(z)\left\langle\gamma_{0}^{n+1} b_{0} \prod_{i=1}^{2 n+1}\left(\beta\left(z_{i}+w\right) c\left(z_{i}+w\right)-b\left(z_{i}+w\right)\right)\right\rangle_{\mathrm{NS}}^{q ;+} \\
& =(q)_{n+1}(-1)^{n+1} \prod_{i=1}^{2 n+1}\left(z_{i}+w\right)^{-1} \sum_{i=0}^{n+1} w^{n+1-i} c_{i}^{(2 n+1)} \mathrm{P}_{\left[2 n+1-\epsilon^{(2 n+1)}(i)\right]}^{-3}\left(z^{-1}\right) \\
& \prod_{i=1}^{2 n+1} z_{i}^{-2 n-1} \Delta(z)\left\langle\gamma_{0}^{n} c_{0} \prod_{i=1}^{2 n+1}\left(\beta\left(z_{i}+w\right) c\left(z_{i}+w\right)-b\left(z_{i}+w\right)\right)\right\rangle_{\mathrm{NS}}^{q ;-} \\
& =(q)_{n}(-1)^{n+1} \prod_{i=1}^{2 n+1}\left(z_{i}+w\right)^{-1} \sum_{i=0}^{n} w^{n+1-i} c_{i}^{(2 n+1)} \mathrm{P}_{\left[2 n+1-\epsilon^{(2 n+1)}(i)\right]}^{-3}\left(z^{-1}\right)
\end{aligned}
$$


where the $c_{i}^{(k)} \in \mathbb{C}$ are non-zero constants.

(2) In the Ramond sector,

$$
\begin{aligned}
& \prod_{i=1}^{2 n} z_{i}^{-2 n+2} \Delta(z)\left\langle\gamma_{0}^{n} \prod_{i=1}^{2 n}\left(\beta\left(z_{i}+w\right) c\left(z_{i}+w\right)-b\left(z_{i}+w\right)\right)\right\rangle_{\mathbb{R}}^{q} \\
& =(q)_{n}(-2)^{n} \prod_{i=1}^{2 n}\left(z_{i}+w\right)^{-\frac{1}{2}} \mathrm{P}_{\delta^{(2 n)}(0,0)}^{-3}\left(z^{-1}\right) \\
& \prod_{i=1}^{2 n+1} z_{i}^{-2 n+1} \Delta(z)\left\langle\gamma_{0}^{n} \prod_{i=1}^{2 n+1}\left(\beta\left(z_{i}+w\right) c\left(z_{i}+w\right)-b\left(z_{i}+w\right)\right) \cdot c(w)\right\rangle_{\mathbb{R}}^{q} \\
& =-(q)_{n}(-2)^{n} w^{\frac{1}{2}} \prod_{i=1}^{2 n+1}\left(z_{i}+w\right)^{-\frac{1}{2}} \mathrm{P}_{\delta^{(2 n+1)(0,0)}}^{-3}\left(z^{-1}\right)
\end{aligned}
$$

Proof. The main stepping stones to the above identities are the identities, proved in Proposition 3.8 of [34],

$$
\begin{aligned}
& \Delta\left(z_{1}, \ldots, z_{2 n}\right) \operatorname{pf}\left(\frac{1}{z_{i}-z_{j}}\right)_{1 \leq i<j \leq 2 n}=\mathrm{P}_{\delta^{(2 n)}(0,0)}^{-3}\left(z_{1}, \ldots, z_{2 n}\right), \\
& \Delta\left(z_{1}, \ldots, z_{2 n}\right) \operatorname{pf}\left(\frac{z_{i}+z_{j}}{z_{i}-z_{j}}\right)_{1 \leq i<j \leq 2 n}=\mathrm{P}_{\delta^{(2 n)}(1,0)}^{-3}\left(z_{1}, \ldots, z_{2 n}\right)
\end{aligned}
$$

the translation invariance of $\mathrm{P}_{\delta^{(2 n)}(0,0)}^{-3}$, that is,

$$
\mathrm{P}_{\delta^{(2 n)}(0,0)}^{-3}\left(z_{1}+w, \ldots, z_{2 n}+w\right)=\mathrm{P}_{\delta^{(2 n)}(0,0)}^{-3}\left(z_{1}, \ldots, z_{2 n}\right)
$$

and the Taylor expansion

$$
\mathrm{P}_{\delta^{(2 n)}(1,0)}^{-3}\left(z_{1}+w, \ldots, z_{2 n}+w\right)=\sum_{i=0}^{n} c_{i}^{(2 n)} \mathrm{P}_{\epsilon^{(2 n)}(i)}^{-3}\left(z_{1}, \ldots, z_{2 n}\right) w^{n-i}
$$

The non-vanishing of the expansion coefficients $c_{i}^{(2 n)}$ was shown in the Remark directly after Proposition 3.9 of [34]. Verifying the above formulae for correlation functions now follows in exactly the same way as it did in Proposition 3.9 of [34], so we give the details for first two cases of (32) only and leave the rest as an exercise for the reader.

When multiplying out the product $\prod_{i=1}^{2 n}\left(\beta\left(z_{i}\right) c\left(z_{i}\right)-b\left(z_{i}\right)\right)$ in

$$
\left\langle\gamma_{0}^{n} \prod_{i=1}^{2 n}\left(\beta\left(z_{i}\right) c\left(z_{i}\right)-b\left(z_{i}\right)\right)\right\rangle_{\mathrm{NS}}^{q ; \pm}
$$

the only summands which can contribute are those containing an equal number of $b$ and $c$ fields, that is, $n$ of each. These summands will also contain $n \beta$ fields and 
so applying the second identity of Theorem 21 implies

$$
\begin{aligned}
\left\langle\gamma_{0}^{n} \prod_{i=1}^{2 n}\left(\beta\left(z_{i}\right) c\left(z_{i}\right)-b\left(z_{i}\right)\right)\right\rangle_{\mathrm{NS}}^{q ; \pm} & =(q)_{n}\left\langle\prod_{i=1}^{2 n}\left(c\left(z_{i}\right) z_{i}^{-1}-b\left(z_{i}\right)\right)\right\rangle_{\mathrm{NS}}^{ \pm} \\
& =(q)_{n}(-1)^{n} \operatorname{pf}\left(\frac{z_{i}^{-1}+z_{j}^{-1}}{z_{i}-z_{j}}\right)_{1 \leq i<j \leq 2 n} \\
& =(q)_{n}(-1)^{n} \prod_{i=1}^{2 n} z_{i}^{-1} \operatorname{pf}\left(\frac{z_{i}+z_{j}}{z_{i}-z_{j}}\right)_{1 \leq i<j \leq 2 n}
\end{aligned}
$$

where the second equality follows from the identities of Theorem 22. Multiplying by the Vandermonde determinant and applying the second identity of (34) then gives

$$
\Delta(z)\left\langle\gamma_{0}^{n} \prod_{i=1}^{2 n}\left(\beta\left(z_{i}\right) c\left(z_{i}\right)-b\left(z_{i}\right)\right)\right\rangle_{\mathrm{NS}}^{q ; \pm}=(q)_{n}(-1)^{n} \prod_{i=1}^{2 n} z_{i}^{-1} \mathrm{P}_{\delta^{(2 n)}(1,0)}^{-3}(z)
$$

Finally, translating all variables by $w$ (recall that the Vandermonde determinant is translation invariant), applying the Taylor expansion (35) and multiplying by $\prod_{i=1}^{2 n} z_{i}^{-2 n+1}$ gives

$$
\begin{aligned}
& \prod_{i=1}^{2 n} z_{i}^{-2 n+1} \Delta(z)\left\langle\gamma_{0}^{n} \prod_{i=1}^{2 n}\left(\beta\left(z_{i}+w\right) c\left(z_{i}+w\right)-b\left(z_{i}+w\right)\right)\right\rangle_{\mathrm{NS}}^{q ; \pm} \\
& \quad=(q)_{n}(-1)^{n} \prod_{i=1}^{2 n}\left(z_{i}+w\right)^{-1} \prod_{i=1}^{2 n} z_{i}^{-2 n+1} \mathrm{P}_{\delta^{(2 n)}(1,0)}^{-3}\left(z_{1}+w, \ldots, z_{2 n}+w\right) \\
& \quad=(q)_{n}(-1)^{n} \prod_{i=1}^{2 n}\left(z_{i}+w\right)^{-1} \sum_{i=0}^{n} w^{n-i} c_{i}^{(2 n)} \mathrm{P}_{\left[2 n-1-\epsilon^{(2 n)}(i)\right]}^{-3}\left(z^{-1}\right)
\end{aligned}
$$

The formulae for an odd number of $\beta\left(z_{i}\right) c\left(z_{i}\right)-b\left(z_{i}\right)$ factors follow from the even case by specialisation. Consider

$$
\begin{aligned}
\prod_{i=0}^{2 n+1} & z_{i}^{-2 n-1} \Delta\left(z_{0}, \ldots, z_{2 n+1}\right)\left\langle\gamma_{0}^{n+1} \prod_{i=0}^{2 n+1}\left(\beta\left(z_{i}+w\right) c\left(z_{i}+w\right)-b\left(z_{i}+w\right)\right)\right\rangle_{\mathrm{NS}}^{q ;+} \\
= & \prod_{i=1}^{2 n+1}\left(1-\frac{z_{i}}{z_{0}}\right) \prod_{i=1}^{2 n+1} z_{i}^{-2 n+1} \Delta\left(z_{1}, \ldots, z_{2 n+1}\right) \\
& \cdot\left\langle\gamma_{0}^{n+1}\left(\sum\right)_{\substack{k \geq 0 \\
l \geq 1}} \beta_{k} c_{l}\left(z_{0}+w\right)^{-k-l-1}-\sum_{k \geq 0} b_{k}\left(z_{0}+w\right)^{-k-1}\right. \\
& \left.\cdot \prod_{i=1}^{2 n+1}\left(\beta\left(z_{i}+w\right) c\left(z_{i}+w\right)-b\left(z_{i}+w\right)\right)\right\rangle_{\mathrm{NS}}^{q ;+}
\end{aligned}
$$


Multiplying by $z_{0}$ and then taking the limit $z_{0} \rightarrow \infty$ (or setting $z_{0}^{-1}=0$ gives

$$
\prod_{i=1}^{2 n+1} z_{i}^{-2 n+1} \Delta\left(z_{1}, \ldots, z_{2 n+1}\right)\left\langle\gamma_{0}^{n+1} b_{0} \prod_{i=1}^{2 n+1}\left(\beta\left(z_{i}+w\right) c\left(z_{i}+w\right)-b\left(z_{i}+w\right)\right)\right\rangle_{\mathrm{NS}}^{q ;+}
$$

while multiplying the right-hand side of (36) by $z_{0}$ and then taking the limit $z_{0} \rightarrow \infty$ gives

$$
\begin{gathered}
(q)_{n+1}(-1)^{n+1} \prod_{i=1}^{2 n+1}\left(z_{i}+w\right)^{-1} \sum_{i=0}^{n+1} w^{n+1-i} c_{i}^{(2 n+2)} \mathrm{P}_{\left[2 n+1-\epsilon^{(2 n+2)}(i)\right]}^{-3}\left(0, z_{1}^{-1}, \ldots, z_{2 n+1}^{-1}\right) \\
=(q)_{n+1}(-1)^{n+1} \prod_{i=1}^{2 n+1}\left(z_{i}+w\right)^{-1} \\
\cdot \sum_{i=0}^{n+1} w^{n+1-i} c_{i}^{(2 n+2)} \mathrm{P}_{\left[2 n+1-\epsilon^{(2 n+1)(i)]}\right.}^{-3}\left(z_{1}^{-1}, \ldots, z_{2 n+1}^{-1}\right),
\end{gathered}
$$

where we have used the fact that the $(2 n+2)$ th part of the partitions $[2 n+1-$ $\left.\epsilon^{(2 n+1)}(i)\right]$ is 0 , so setting $z_{0}^{-1}=0$ amounts to dropping the $(2 n+2)$ th part of the partition and considering the corresponding Jack polynomials in $2 n+1$ variables.

\section{The proof of Theorem 10}

In this section we prove Theorem 10 by evaluating the zero mode of the singular vector on suitable candidate relaxed highest weight vectors and thereby calculating the image of the singular vector in the Zhu algebras. The evaluation of these zero modes boils down to evaluating inner products of symmetric polynomials of the form

$$
\left\langle\mathrm{P}_{\kappa}^{-3}(z), \prod_{i=1}^{n}\left(1+\frac{z_{i}}{w}\right)^{\lambda}\right\rangle_{n}^{t}
$$

where $\lambda$ is a complex number depending on free fields weights, $\kappa$ is an admissible partition, so that the Jack polynomial $\mathrm{P}_{\kappa}^{-3}(z)$ is well defined, $t$ is positive rational and the inner product is the integral inner product (26) with respect to which Jack polynomials at parameter value $t$ are orthogonal. In order to evaluate this inner product both the left and right argument need to be expanded in Jack polynomials at parameter value $t$. The right argument expands as, see [25, Eq. (A.28)] with $X=-\lambda t$

$$
\prod_{i=1}^{n}\left(1+\frac{z_{i}}{w}\right)^{\lambda}=\sum_{\mu}(-w)^{|\mu|} \prod_{b \in \mu} \frac{-\lambda t+t a^{\prime}(b)-\ell^{\prime}(b)}{t(a(b)+1)+\ell(b)} \mathrm{P}_{\mu}^{t}(z)
$$

where $\mu$ runs over all partitions of integers of length at most $n, b$ runs over all the cells in the Young diagram of $\mu$, and $a(b), \ell(b), a^{\prime}(b)$ and $\ell^{\prime}(b)$ are the arm length, leg length, arm colength and leg colength, respectively, of $b$. The left argument satisfies an upper triangular decomposition $\mathrm{P}_{\kappa}^{-3}(z)=\mathrm{P}_{\kappa}^{t}(z)+\sum_{\mu<\kappa} c_{\kappa, \mu} \mathrm{P}_{\mu}^{t}(z)$, 
where the $c_{\kappa, \mu}$ are rational functions of $t$ and $\mu<\kappa$ denotes the dominance partial ordering of partitions. The inner product therefore evaluates to

$$
\begin{aligned}
\left\langle\mathrm{P}_{\kappa}^{-3}(z), \prod_{i=1}^{n}\left(1+\frac{z_{i}}{w}\right)^{\lambda}\right\rangle_{n}^{t} & =(-w)^{|\kappa|} \sum_{\mu<\kappa} c_{\mu} \prod_{b \in \mu} \frac{-\lambda t+t a^{\prime}(b)-\ell^{\prime}(b)}{t(a(b)+1)+\ell(b)}, \\
c_{\mu} & =c_{\kappa, \mu}\left\langle\mathrm{P}_{\mu}^{t}(z), \mathrm{P}_{\mu}^{t}(z)\right\rangle_{n}^{t} .
\end{aligned}
$$

Note in particular, that the coefficients $c_{\mu}$ do not depend on the exponent $\lambda$. So the inner product (37) is a polynomial in $\lambda$ and because the numerators of the summands on the right-hand side of (38) depend only on arm and leg colengths, the cells common to all partitions dominated by $\kappa$ will give rise to factors common to the numerators of all summands and hence zeros in the parameter $\lambda$ common to all summands. Finding these common zeros is key to the proofs below and we shall find them using Lemma 3.2 of [34].

\section{The case when $v>u$ and $k<-1$}

Throughout this section we assume that $u, v$ are integers satisfying $v>u \geq 2$, $u-v \in 2 \mathbb{Z}$ and $\operatorname{gcd}(u,(u-v) / 2)$ and that $\xi=\sqrt{u / v}$. We will prove Theorem 10 under the assumption $v>u$ by using the screening operator $\mathcal{Q}_{1}$ to construct the singular vector of $\mathrm{V}_{k_{u, v}}$ and then evaluating the action of its zero mode on relaxed highest weight vectors to deduce its image in the Zhu algebras. Note that for $v>u$, the exponent $\left(\xi^{-2}-1\right) / 2=(v-u) / 2 u$ in $(24)$ is positive rational and will form the Jack polynomial parameter.

Lemma 24. The singular vector of $\mathrm{V}_{k_{u, v}}$, as a subalgebra of $\mathrm{F}$, is given by

$$
\chi_{u, v}=\mathcal{Q}_{1}^{[u-1]}|(u-1) / \xi ; \mathrm{NS}\rangle
$$

where $|(u-1) / \xi ; \mathrm{NS}\rangle$ is the highest weight vector of $\mathrm{F}_{(u-1) / \xi}^{\mathrm{NS}}$.

To prove this lemma we consider both symmetric polynomials and their infinitevariable limits, the symmetric functions. For easy visual distinction, we denote the infinite alphabet of variables for symmetric functions by $y=\left(y_{1}, y_{2}, \ldots\right)$ and the finite alphabet of variables for symmetric polynomials by $z=\left(z_{1}, \ldots, z_{n}\right)$. We shall use both the infinite- and finite-variable inner products $\langle\cdot, \cdot\rangle^{t}$ and $\langle\cdot, \cdot\rangle_{n}^{t}$, referring to $[25$, App. A] for our conventions, as well as the identity (see [25, Eq. (A.16)])

$$
\prod_{m \geq 1} \exp \left(\frac{1}{t} \frac{\mathrm{p}_{m}(z) \mathrm{p}_{m}(y)}{m}\right)=\sum_{\lambda} \mathrm{P}_{\lambda}^{t}(z) \mathrm{Q}_{\lambda}^{t}(y)
$$

where $\mathrm{p}_{m}$ is the $m$-th power sum and the $\mathrm{Q}_{\lambda}^{t}(y)$ are the symmetric functions dual (with respect to $\langle\cdot, \cdot\rangle^{t}$ ) to the Jack symmetric functions $\mathrm{P}_{\lambda}^{t}(y)$.

A simple, but very useful, observation concerning the ring of symmetric functions $\Lambda$ is that it is isomorphic, as a commutative algebra, to the universal enveloping of either the positive or negative subalgebra, $\mathfrak{H}_{1}^{+}$or $\mathfrak{H}_{1}^{-}$, of the rank 1 Heisenberg algebra $\mathfrak{H}_{1}$. We denote the corresponding isomorphisms by 


$$
\begin{aligned}
\rho_{\gamma}^{+}: \Lambda & \rightarrow \mathbb{C}\left[a_{1}, a_{2}, \ldots\right], & \rho_{\gamma}^{-}: \Lambda & \rightarrow \mathbb{C}\left[a_{-1}, a_{-2}, \ldots\right], \\
\mathrm{p}_{m} & \mapsto \gamma a_{m}, & \mathrm{p}_{m} & \mapsto \gamma a_{-m},
\end{aligned}
$$

We shall use these isomorphisms to identify inner products involving Heisenberg generators with the symmetric function inner product $\langle\cdot, \cdot\rangle^{t}$. For example, one easily verifies in the power sum basis, hence for arbitrary $f, g \in \Lambda$, that

$$
\langle f, g\rangle^{t}=\left\langle q\left|\rho_{t / \gamma}^{+}(f) \rho_{\gamma}^{-}(g)\right| q\right\rangle
$$

where the right-hand side is evaluated in the Fock space $\mathbb{F}_{q}$, for any $q \in \mathbb{C}$ and any $\gamma \in \mathbb{C} \backslash\{0\}$.

Proof. Since $|(u-1) \sqrt{v / u} ; \mathrm{NS}\rangle$ is an $\widehat{\mathfrak{o s p}}(1 \mid 2)$ highest weight vector and $\mathcal{Q}_{1}^{[u-1]}$ is a module homomorphism, the image of $\mid(u-1) \sqrt{v / u}$; NS $\rangle$ must be either a singular vector or zero. By Theorem 17 the singular vector in $\mathrm{F}$ is unique and therefore it is sufficient to show that $\mathcal{Q}_{1}^{[u-1]} \mid(u-1) \sqrt{v / u}$; NS $\rangle$ is non-zero. We do this by explicitly evaluating certain matrix elements and verifying that they are non-zero.

Evaluating formula $(24)$ for $\mathcal{Q}_{1}^{[u-1]}$ on $|(u-1) / \xi ; \mathrm{NS}\rangle$ yields

$$
\begin{aligned}
\mathcal{Q}_{1}^{[u-1]}|(u-1) / \xi ; \mathrm{NS}\rangle=\int \prod_{i \neq j}\left(1-\frac{z_{i}}{z_{j}}\right)^{\left(\xi^{-2}-1\right) / 2} \\
\cdot \prod_{i=1}^{u-1} z_{i}^{2-(u+v) / 2} \Delta(z)\left(\beta\left(z_{1}\right) c\left(z_{1}\right)-b\left(z_{1}\right)\right) \cdots\left(\beta\left(z_{u-1}\right) c\left(z_{u-1}\right)-b\left(z_{u-1}\right)\right) \\
\cdot \prod_{m \geq 1} \exp \left(\frac{-1}{\xi} \frac{a_{-m}}{m} \mathrm{p}_{m}(z)\right)|0 ; \mathrm{NS}\rangle \frac{\mathrm{d} z_{1} \cdots \mathrm{d} z_{u-1}}{z_{1} \cdots z_{u-1}} .
\end{aligned}
$$

To further evaluate this formula, we distinguish between $u$ and $v$ even or odd.

Suppose first that $u$ and $v$ are odd. Then, by the identities (31) in Theorem 23,

$$
\begin{aligned}
& \prod_{i=1}^{u-1} z_{i}^{2-(u+v) / 2} \Delta(z)\left\langle\frac{\gamma_{1}^{(u-1) / 2}}{(u-1) / 2 !}\left(\beta\left(z_{1}\right) c\left(z_{1}\right)-b\left(z_{1}\right)\right) \cdots\left(\beta\left(z_{u-1}\right) c\left(z_{u-1}\right)-b\left(z_{u-1}\right)\right)\right\rangle_{\mathrm{NS}}^{+} \\
& =\prod_{i=1}^{u-1} z_{i}^{(u-v) / 2-1} \mathrm{P}_{\delta^{(u-1)}(0,0)}^{-3}\left(z^{-1}\right)=\mathrm{P}_{\kappa}^{-3}\left(z^{-1}\right),
\end{aligned}
$$

where $\kappa$ is the admissible partition $\kappa=\delta^{(u-1)}((v-u) / 2+1,(v-u) / 2+1)$ and \langle\rangle$_{\mathrm{NS}}^{+}$denotes the combination of the $\beta \gamma$ and $b c$ correlation functions. The nonvanishing of $\mathcal{Q}_{1}^{[u-1]} \mid(u-1) / \xi$; NS $\rangle$ then follows by evaluating the following matrix 
element as an integral of correlators:

$$
\begin{gathered}
\left\langle 0 ; \mathrm{NS}\left|\frac{\gamma_{1}^{(u-1) / 2}}{(u-1) / 2 ! 2^{(u-1) / 2}} \rho_{-\xi}^{+}\left(\mathrm{P}_{\kappa}^{2 /\left(\xi^{-2}-1\right)}(y)\right) \mathcal{Q}_{1}^{[u-1]}\right|(u-1) / \xi ; \mathrm{NS}\right\rangle \\
=\int \prod_{i \neq j}\left(1-\frac{z_{i}}{z_{j}}\right)^{\left(\xi^{-2}-1\right) / 2} \mathrm{P}_{\kappa}^{-3}\left(z^{-1}\right) \mathrm{P}_{\kappa}^{2 /\left(\xi^{-2}-1\right)}(z) \frac{\mathrm{d} z_{1} \cdots \mathrm{d} z_{u-1}}{z_{1} \cdots z_{u-1}} \\
=\left\langle\mathrm{P}_{\kappa}^{-3}(z), \mathrm{P}_{\kappa}^{2 /\left(\xi^{-2}-1\right)}(z)\right\rangle_{u-1}^{2 /\left(\xi^{-2}-1\right)} \\
=\left\langle\mathrm{P}_{\kappa}^{2 /\left(\xi^{-2}-1\right)}(z), \mathrm{P}_{\kappa}^{2 /\left(\xi^{-2}-1\right)}(z)\right\rangle_{u-1}^{2 /\left(\xi^{-2}-1\right)} \neq 0
\end{gathered}
$$

where the third equality uses the fact that Jack symmetric polynomials decompose upper triangularly (with leading coefficient equal to 1) in terms of Jack symmetric polynomials at different values of the parameter and that Jack symmetric polynomials at parameter value $2 /\left(\xi^{-2}-1\right)$ are orthogonal with respect to the inner product $\langle,\rangle_{u-1}^{2 /\left(\xi^{-2}-1\right)}$.

The case where $u$ and $v$ are even follows similarly by evaluating the matrix element

$$
\begin{aligned}
&\left\langle 0 ; \mathrm{NS}\left|\frac{\gamma_{1}^{(u-2) / 2}}{u / 2 ! 2^{u / 2}} b_{0} \rho_{-\xi}^{+}\left(\mathrm{P}_{\kappa}^{2 /\left(\xi^{-2}-1\right)}(y)\right) \mathcal{Q}_{1}^{[u-1]}\right|(u-1) / \xi ; \mathrm{NS}\right\rangle \\
&=\left\langle\mathrm{P}_{\kappa}^{-3}(z), \mathrm{P}_{\kappa}^{2 /\left(\xi^{-2}-1\right)}(z)\right\rangle_{u-1}^{2 /\left(\xi^{-2}-1\right)} \\
&=\left\langle\mathrm{P}_{\kappa}^{2 /\left(\xi^{-2}-1\right)}(z), \mathrm{P}_{\kappa}^{2 /\left(\xi^{-2}-1\right)}(z)\right\rangle_{u-1}^{2 /\left(\xi^{-2}-1\right)} \neq 0
\end{aligned}
$$

where $\kappa=\delta^{(u-1)}((v-u) / 2+2,(v-u) / 2)$.

Lemma 25. Up to an irrelevant scale factor, the polynomial $g(h, \Sigma)$ of Theorem 9 is given by

$$
g(h, \Sigma)=\prod_{(i, j) \in \mathrm{K}_{u, v}^{\mathrm{NS}}}\left(\Sigma-s_{i, j}\right), \quad s_{i, j}=\frac{i}{2}-\frac{u j}{2 v} .
$$

In particular, $g(h, \Sigma)$ does not depend on $h$.

Proof. As stated in Theorem 9, the degree of the polynomial $g(h, \Sigma)$ is bounded by $(u-1)(v-1) / 2$. We shall show that the factors $\left(\Sigma-s_{i, j}\right)$ of $(41)$ divide $g(h, \Sigma)$ by showing that $g\left(h, s_{i, j}\right)$ vanishes independently of $h$. Since these factors saturate the maximal degree of $g$ and $g$ is non-zero by Theorem 8 , they uniquely determine $g$ up to scaling.

Consider first the case when $u$ and $v$ are odd. The zero mode of the singular vector maps the relaxed highest weight vectors $\mid p ; q$; NS $\rangle$ and $c_{0} \mid p ; q$; NS $\rangle$ to multiples of $\beta_{0}^{(u-1) / 2}|p ; q ; \mathrm{NS}\rangle$ and $\beta_{0}^{(u-1) / 2} c_{0}|p ; q ; \mathrm{NS}\rangle$, respectively. This mapping is 
non-zero if and only if the following matrix elements are also non-zero.

$$
\begin{aligned}
\left\langle p ; q ; \mathrm{NS}\left|\gamma_{0}^{(u-1) / 2} \mathcal{Q}_{1}^{[u-1]} \mathrm{V}_{((u-1) / \xi) a}(w)\right| p ; q ; \mathrm{NS}\right\rangle & \\
= & \left\langle\left\langle\gamma_{0}^{(u-1) / 2} \prod_{i=1}^{u-1}\left(\beta\left(z_{i}+w\right) c\left(z_{i}+w\right)-b\left(z_{i}+w\right)\right)\right\rangle_{\mathrm{NS}}^{q ; \pm}\right. \\
& \left\langle p a\left|\prod_{i=1}^{u-1} \mathrm{~V}_{-a / \xi}\left(z_{i}+w\right) \cdot \mathrm{V}_{((u-1) / \xi) a}(w)\right| p a\right\rangle \mathrm{d} z_{1} \cdots \mathrm{d} z_{u-1} \\
= & \prod_{i \neq j}\left(1-\frac{z_{i}}{z_{j}}\right)^{\left(\xi^{-2}-1\right) / 2} \prod_{i=1}^{u-1}\left(1+\frac{z_{i}}{w}\right)^{-p / \xi} \prod_{i=1}^{u-1} z_{i}^{2-(u+v) / 2} \\
& \Delta(z)\left\langle\gamma_{0}^{(u-1) / 2} \prod_{i=1}^{u-1}\left(\beta\left(z_{i}+w\right) c\left(z_{i}+w\right)-b\left(z_{i}+w\right)\right)\right\rangle_{\mathrm{NS}}^{q ; \pm} \frac{\mathrm{d} z_{1} \cdots \mathrm{d} z_{u-1}}{z_{1} \cdots z_{u-1}} \\
= & w^{(1-u) / 2} \sum_{i=0}^{(u-1) / 2} w^{-i} c_{i}\left\langle\mathrm{P}_{\left[(v+u) / 2-2-\epsilon^{(u-1)}(i)\right]}^{-3}(z), \prod_{i=1}^{u-1}\left(1+\frac{z_{i}}{w}\right)^{-p / \xi-1}\right\rangle_{u-1}^{2 /\left(\xi^{-2}-1\right)}
\end{aligned}
$$

the second equality follows from the identities (32) in Theorem 23. By the argument outlined at the beginning of Section 4 , we need to identify the cells common to all partitions dominated by $\left[(v+u) / 2-2-\epsilon^{(u-1)}(i)\right], i=0, \ldots,(u-1) / 2$. The parts of the admissible partitions $\left[(v+u) / 2-2-\epsilon^{(u-1)}(i)\right]$ are all bounded below by the parts of $\left[(v+u) / 2-2-\delta^{(u-1)}(1,0)\right]=\delta^{(u-1)}((v-u) / 2+1,(v-u) / 2)$ and so by $[34$, Lem. 3.2.(3)], the cells common to all partitions dominated by the $\left[(v+u) / 2-2-\epsilon^{(u-1)}(i)\right]$ form the length $u-1$ partition

$$
\rho=\left[\frac{v-1}{2}, \frac{v-1}{2}-1, \frac{v-1}{2}-1, \ldots, \frac{v-u}{2}+1, \frac{v-u}{2}+1, \frac{v-u}{2}\right],
$$

that is, $\rho_{1}=(v-1) / 2=\rho_{2}+1$ and $\rho_{i}=\rho_{i-2}+1$ for $i=3, \ldots, u-1$. Therefore, by (38), the zeros of

$$
\prod_{b \in \rho}\left(\frac{2}{\xi^{-2}-1}\left(\frac{p}{\xi}+1\right)+\frac{2}{\xi^{-2}-1} a^{\prime}(b)-\ell^{\prime}(b)\right)
$$

are common to all the summands of (42). Up to irrelevant scale factors, this polynomial can be rewritten as

$$
\begin{aligned}
& \prod_{b \in \rho}\left(\xi p+\frac{1}{2}-\frac{1}{2}\left(\ell^{\prime}(b)+1\right)+\frac{\xi^{2}}{2}\left(\ell^{\prime}+2 a^{\prime}(b)+2\right)\right) \\
& =\prod_{b \in \rho}\left( \pm s_{p}-s_{\ell^{\prime}(b)+1, \ell^{\prime}(b)+2 a^{\prime}(b)+2}\right) \\
& =\prod_{j=1}^{(v-1) / 2}\left( \pm s_{p}-s_{1,2 j}\right) \prod_{i=1}^{(u-3) / 2} \prod_{j=1}^{(v-1) / 2-i}\left( \pm s_{p}-s_{2 i, 2 i+2 j-1}\right)\left( \pm s_{p}-s_{2 i+1,2 i+2 j}\right) \\
& \cdot \prod_{j=1}^{(v-u) / 2}\left( \pm s_{p}-s_{u-1, u+2 j-2}\right)
\end{aligned}
$$


where we recall that $s_{p}= \pm\left(\xi p+\frac{1}{2}\right)$ are the respective eigenvalues of $\Sigma$ acting on the relaxed highest weight vectors $|p ; q ; \mathrm{NS}\rangle$ and $c_{0}|p ; q ; \mathrm{NS}\rangle$. So for $(i, j) \in \mathrm{K}_{u, v}^{\mathrm{NS}}$, $i<j$ we have that $g\left(h, s_{i, j}\right)=0$ and $g\left(h,-s_{i, j}\right)=0$. Since $-s_{i, j}=s_{u-i, v-j}$, this implies $g\left(h, s_{i, j}\right)=0$ for all $(i, j) \in \mathrm{K}_{u, v}^{\mathrm{NS}}$. Note we have used the fact that $v>u$, otherwise the ordered pairs $(i, j),(u-i, v-j)$, with $i<j$, would not cover the entire Neveu-Schwarz Kac table.

Next, consider the case of even $u$ and $v$. The action of the zero mode of the singular vector acting on the relaxed highest weight vectors $|p ; q ; \mathrm{NS}\rangle$ and $c_{0}|p ; q ; \mathrm{NS}\rangle$ is non-zero if and only if the following matrix elements are also nonzero:

$$
\begin{gathered}
\left\langle p ; q ; \mathrm{NS}\left|\gamma_{0}^{u / 2} b_{0} \mathcal{Q}_{1}^{[u-1]} \mathrm{V}_{(u-1) / \xi}(w)\right| p ; q ; \mathrm{NS}\right\rangle \\
\left\langle p ; q ; \mathrm{NS}\left|b_{0} \gamma_{0}^{(u-2) / 2} c_{0} \mathcal{Q}_{1}^{[u-1]} \mathrm{V}_{(u-1) / \xi}(w) c_{0}\right| p ; q ; \mathrm{NS}\right\rangle .
\end{gathered}
$$

Up to irrelevant factors involving $q$, both matrix elements evaluate to

$$
w^{3 u / 2-2} \sum_{i=1}^{u / 2-1} w^{-i} c_{i}^{(u-1)}\left\langle\mathrm{P}_{[(v+u) / 2-2-\epsilon(u-1)(i)]}^{-3}(z), \prod_{i=1}^{u-1}\left(1+\frac{z_{i}}{w}\right)^{-p / \xi-1}\right\rangle_{u-1}^{2 /\left(\xi^{-2}-1\right)} .
$$

As in the case of odd $u, v$ we identify zeros by finding cells common to all Young diagrams of partitions dominated by $\left[(v+u) 2-2-\epsilon^{(u-1)}(i)\right]$. By [34, Lem. 3.2.(4)],

$$
\rho=\left[\frac{v}{2}-1, \frac{v}{2}-1, \frac{v}{2}-2, \frac{v}{2}-2, \ldots, \frac{v-u}{2}+1, \frac{v-u}{2}+1, \frac{v-u}{2}\right]
$$

is the partition of length $u-1$ formed by these cells. The zeros of

$$
\begin{aligned}
& \prod_{b \in \rho}\left(\xi p+\frac{1}{2}-\frac{1}{2}\left(\ell^{\prime}(b)+1\right)+\frac{\xi^{2}}{2}\left(\ell^{\prime}+2 a^{\prime}(b)+2\right)\right) \\
& \quad=\prod_{b \in \rho}\left( \pm s_{p}-s_{\ell^{\prime}(b)+1, \ell^{\prime}(b)+2 a^{\prime}(b)+2}\right) \\
& \quad=\prod_{i=1}^{u / 2-1} \prod_{j=1}^{v / 2-i}\left( \pm s_{p}-s_{2 i-1,2 i+2 j-2}\right)\left( \pm s_{p}-s_{2 i, 2 i+2 j-1}\right) \prod_{j=1}^{(v-u) / 2}\left( \pm s_{p}-s_{u-1, u+2 j-2}\right) .
\end{aligned}
$$

are therefore also zeros of the original matrix elements (44). As in the case for $u$ and $v$ odd, this implies that $g\left(h, s_{i, j}\right)=0$ for all $(i, j) \in \mathrm{K}_{u, v}^{\mathrm{NS}}$.

Lemma 26. Up to an irrelevant scale factor, the polynomial $g_{\tau}(h, Q)$ of Theorem 9 is given by

$$
g_{\tau}(h, Q)=\prod_{[(i, j)] \in \overline{\mathrm{K}}_{u, v}^{\mathrm{R}}}\left(Q-q_{i, j}\right), \quad q_{i, j}=\frac{(u j-v i)^{2}-4 v^{2}}{8 v^{2}} .
$$

In particular, $g_{\tau}(h, Q)$ does not depend on $h$. 
Proof. As stated in Theorem 9, the degree of the polynomial $g_{\tau}(h, Q)$ is bounded by $((u-1)(v-1)-1) / 2$. We shall show that the factors $\left(Q-q_{i, j}\right)$ of $(45)$ divide $g_{\tau}(h, Q)$ by showing that $g_{\tau}$ vanishes independently of $h$ at appropriate values of $Q$. Since these factors saturate the maximal degree of $g_{\tau}$ and $g_{\tau}$ is non-zero by Theorem 8 , they uniquely determine $g_{\tau}$ up to scaling.

Consider first the case where $u$ and $v$ are odd. The zero mode of the singular vector maps the relaxed highest weight vector $|p ; j ; \mathrm{R}\rangle$ to a multiple of the vector $\beta_{0}^{(u-1) / 2}|p ; j ; \mathrm{R}\rangle$. This action is non-zero if and only if the following matrix element is non-zero:

$$
\begin{aligned}
& \left\langle p ; j ; \mathrm{R}\left|\gamma_{0}^{(u-1) / 2} \mathcal{Q}_{1}^{[u-1]} \mathrm{V}_{((u-1) / \xi) a}(w)\right| p ; j ; \mathrm{R}\right\rangle \\
& =\int \prod_{i=1}^{u-1}\left(1-\frac{z_{i}}{z_{j}}\right)^{\left(\xi^{-2}-1\right) / 2} \prod_{i=1}^{u-1}\left(1+\frac{z_{i}}{w}\right)^{-1} \\
& \quad \cdot \prod_{i=1}^{u-1} z_{i}^{2-(u+v) / 2} \Delta(z)\left\langle\gamma_{0}^{(u-1) / 2} \prod_{i=1}^{u-1}\left(\beta\left(z_{i}\right) c\left(z_{i}\right)-b\left(z_{i}\right)\right)\right\rangle_{\mathrm{R}}^{j} \frac{\mathrm{d} z_{1} \cdots \mathrm{d} z_{u-1}}{z_{1} \cdots z_{u-1}}
\end{aligned}
$$

Using the identities (33) of Theorem 23, this evaluates to

$$
(j)_{(u-1) / 2}(-2)^{(u-1) / 2}\left\langle\mathrm{P}_{\delta^{(u-1)}((v-u) / 2+1,(v-u) / 2+1)}^{-3}(z), \prod_{i=1}^{u-1}\left(1+\frac{z_{i}}{w}\right)^{-p / \xi-1 / 2}\right\rangle_{u-1}^{2 /\left(\xi^{-2}-1\right)} .
$$

As in the proof of Theorem 25, cells common to all Young diagrams of partitions dominated by $\delta^{(u-1)}((v-u) / 2+1,(v-u) / 2+1)$ can be used to identify zero of the above inner product. By [34, Lem. 3.2.(1)] these cells form the length $u-1$ partition

$$
\rho=\left[\frac{v-1}{2}, \frac{v-1}{2}, \frac{v-1}{2}-1, \frac{v-1}{2}-1, \ldots, \frac{v-u}{2}+1, \frac{v-u}{2}+1\right]
$$

and the zeros of (46) include the zeros of

$$
\begin{aligned}
\prod_{b \in \rho}\left(\xi p+\frac{\xi^{2}}{2}\right. & \left.+\xi^{2} a^{\prime}(b)-\frac{1-\xi^{2}}{2} \ell^{\prime}(b)\right) \\
& =\prod_{b \in \rho}\left(\xi p+\frac{1}{2}-\frac{1}{2}\left(\ell^{\prime}(b)+1\right)+\frac{\xi^{2}}{2}\left(\ell^{\prime}(b)+2 a^{\prime}(b)+1\right)\right) \\
& =\prod_{b \in \rho}\left(s_{p}-s_{\ell^{\prime}(b)+1, \ell^{\prime}(b)+2 a^{\prime}(b)+1}\right)=\prod_{\substack{(i, j) \in \mathrm{K}_{u, v}^{\mathrm{R}} \\
i \leq j}}\left(s_{p}-s_{i, j}\right) .
\end{aligned}
$$

Since the above zeros do not depend on the $\beta \gamma$ weight, they correspond to values of $Q$ at which the polynomial $g_{\tau}(h, Q)$ vanishes independently of $h$. Recall that $q_{p}=\frac{1}{2}\left(s_{p}^{2}-1\right)$ and assume $g_{\tau}(h, d)=0$ for some $d \in \mathbb{C}$ independent of $h$; then $g_{\tau}\left(h, q_{p}\right)$ is divided by

$$
q_{p}-d=\frac{1}{2}\left(s_{p}^{2}-1\right)-d=\frac{1}{2}\left(s_{p}-\tilde{d}\right)\left(s_{p}+\tilde{d}\right),
$$


where $\pm \tilde{d}$ are the square roots of $2 d+1$. So if $\left(s_{p}-s_{i, j}\right)$ divides $g\left(h, q_{p}\right)$, then $\left(s_{p}+s_{i, j}\right)$ must do so also. Thus the product $\left(s_{p}-s_{i, j}\right)\left(s_{p}+s_{i, j}\right)=\left(2 q_{p}-s_{i, j}^{2}+1\right)$ $=2\left(q_{p}-q_{i, j}\right)$ divides $g\left(h, q_{p}\right)$. So since $Q-q_{i, j}$ divides $g_{\tau}$ for all $i \leq j,(i, j) \in \mathrm{K}_{u, v}^{\mathrm{R}}$ and every class in $\overline{\mathrm{K}}_{u, v}^{\mathrm{R}}$ has a representative of this form (assuming $v>u$ ), this proves the lemma for $u$ and $v$ odd.

Next consider the case when $u$ and $v$ are even; then the action of the zero mode of the singular vector on a relaxed highest weight vector $|p ; j ; \mathrm{R}\rangle$ is non-zero if and only if the following matrix element is non-zero:

$$
\begin{aligned}
& \left\langle p ; j ; \mathrm{R}\left|\gamma_{0}^{u / 2-1} \mathcal{Q}_{1}^{[u-1]} c(w) \mathrm{V}_{((u-1) / \xi) a}(w)\right| p ; j ; \mathrm{R}\right\rangle \\
& =\int \prod_{i=1}^{u-1}\left(1-\frac{z_{i}}{z_{j}}\right)^{\left(\xi^{-2}-1\right) / 2} \prod_{i=1}^{u-1} z_{i}^{2-(u+v) / 2} \Delta(z) \\
& \quad \cdot\left\langle\gamma_{0}^{(u-2) / 2} \prod_{i=1}^{u-1}\left(\beta\left(z_{i}+w\right) c\left(z_{i}+w\right)-b\left(z_{i}+w\right)\right) c(w)\right\rangle_{\mathrm{R}}^{j} \frac{\mathrm{d} z_{1} \cdots \mathrm{d} z_{u-1}}{z_{1} \cdots z_{u-1}} .
\end{aligned}
$$

Up to an irrelevant scale factor in $j$ this evaluates to

$$
\left\langle\mathrm{P}_{\delta^{(u-1)}((v-u) / 2+1,(v-u) / 2+1)}^{-3}(z), \prod_{i=1}^{u-1}\left(1+\frac{z_{i}}{w}\right)^{-p / \xi-1 / 2}\right\rangle_{u-1}^{2 /\left(\xi^{-2}-1\right)} .
$$

By [34, Lem. 3.2.(2)], the partition formed by the cells common to the Young diagrams of all partitions dominated by $\delta^{(u-1)}\left(\frac{v-u}{2}+1, \frac{v-u}{2}+1\right)$ is

$$
\rho=\left[\frac{v}{2}-1, \frac{v}{2}-2, \frac{v}{2}-2, \ldots, \frac{v-u}{2}+1, \frac{v-u}{2}+1\right] .
$$

Thus, the zeros of the matrix element (47) include those of

$$
\prod_{b \in \rho}\left(s_{p}-s_{\ell^{\prime}(b)+1, \ell^{\prime}(b)+2 a^{\prime}(b)+1}\right)=\prod_{\substack{(i, j) \in \mathrm{K}_{u, v}^{\mathrm{R}} \\ i \leq j}}\left(s_{p}-s_{i, j}\right) .
$$

The remainder of the proof then follows as in the case of $u$ and $v$ odd.

\section{The case when $u>v$ and $k>-1$}

Throughout this section we will assume that $u, v$ are integers satisfying $u>v \geq 1$, $u-v \in 2 \mathbb{Z}$ and $\operatorname{gcd}(u,(u-v) / 2)$ and that $\xi=\sqrt{u / v}$. We will prove Theorem 10 under the assumption $u>v$ by using the screening operator $\mathcal{Q}_{2}$ to construct the singular vector of $\mathrm{V}_{k_{u, v}}$ and then evaluating the action of its zero mode on relaxed highest weight vectors to deduce its image on the Zhu algebras. Note that for $u>v$, the exponent $\left(\xi^{2}-1\right) / 2=(u-v) / 2 v$ in (25) is positive rational and will form the Jack polynomial parameter. Throughout this section we will be using the second free field realisation of $\mathrm{V}_{k}$ in which $\beta(z)=\mathrm{V}_{\theta+\psi}(z)$. 
Lemma 27. The singular vector of $\mathrm{V}_{k_{u, v}}$, as a subalgebra of $\mathrm{B}$, is given by

$$
\chi_{u, v}=\mathcal{Q}_{2}^{[v-1]}\left|(1-v) \xi ;\left(u-\frac{u+v}{2 v}\right)(\theta+\psi) ; \mathrm{NS}\right\rangle
$$

where $|(1-v) \xi ;(u-(u+v) / 2 v)(\theta+\psi) ; \mathrm{NS}\rangle$ is the highest weight vector of

$$
\mathbb{F}_{(1-v) \xi a} \otimes \mathbb{F}_{(u-(u+v) / 2 v)(\theta+\psi)} \otimes \mathbb{F}^{\mathrm{NS}}
$$

Proof. Since the singular vector is unique by Theorem 18, it suffices to show that (48) does not vanish. As in the previous section, we do this by verifying that certain matrix elements are non-zero.

Expanding (48) as an integral of multiple copies of the screening field $\mathcal{Q}_{2}(z)$ gives

$$
\begin{aligned}
\int & \prod_{i \neq j}\left(1-\frac{z_{i}}{z_{j}}\right)^{\left(\xi^{2}-1\right) / 2} \prod_{i=1}^{v-1} z_{i}^{2-(u+v) / 2} \Delta(z) \\
& \prod_{i=1}^{v-1}\left(\beta\left(z_{i}\right) c\left(z_{i}\right)-b\left(z_{i}\right)\right) \prod_{i=1}^{v-1} \mathrm{~V}_{-((u+v) / 2 v)(\theta+\psi)}\left(z_{i}\right) \\
& \cdot \prod_{m \geq 1} \exp \left(\xi \frac{a_{-m}}{m} \mathrm{p}_{m}(z)\right)\left|0 ;\left(u-\frac{u+v}{2 v}\right)(\theta+\psi) ; \mathrm{NS}\right\rangle \frac{\mathrm{d} z_{1} \cdots \mathrm{d} z_{v-1}}{z_{1} \cdots z_{v-1}}
\end{aligned}
$$

For $u$ and $v$ odd, we show that this is non-zero by computing the matrix element

$$
\left\langle 0 ; \frac{u-1}{2}(\theta+\psi) ; \mathrm{NS}\left|\rho_{\xi^{-1}}^{+}\left(\mathrm{P}_{\kappa}^{2 /\left(\xi^{2}-1\right)}(y)\right) \mathcal{Q}_{2}^{[v-1]}\right|(1-v) \xi ;\left(u-\frac{u+v}{2 v}\right)(\theta+\psi) ; \mathrm{NS}\right\rangle,
$$

where $\kappa=\delta^{(v-1)}(1+(u-v) / 2,1+(u-v) / 2)$. The above matrix element then evaluates to

$$
\begin{aligned}
& \int \prod_{i \neq j}\left(1-\frac{z_{i}}{z_{j}}\right)^{\left(\xi^{2}-1\right) / 2} \mathrm{P}_{\kappa}^{2 /\left(\xi^{2}-1\right)}(z) \prod_{i=1}^{v-1} z_{i}^{2-(u+v) / 2} \Delta(z) \\
& \cdot\left\langle\frac{\langle-1}{2}(\theta+\psi) ; \mathrm{NS}\right| \prod_{i=1}^{v-1}\left(\beta\left(z_{i}\right) c\left(z_{i}\right)-b\left(z_{i}\right)\right) \\
& \cdot \prod_{i=1}^{v-1} \mathrm{~V}_{-((u+v) / 2 v)(\theta+\psi)}\left(z_{i}\right)\left|\left(u-\frac{u+v}{2 v}\right)(\theta+\psi) ; \mathrm{NS}\right\rangle \frac{\mathrm{d} z_{1} \cdots \mathrm{d} z_{v-1}}{z_{1} \cdots z_{v-1}}
\end{aligned}
$$

The combined $\beta \gamma$ weight of the ket and the $\mathrm{V}_{-((u+v) / 2 v)(\theta+\psi)}\left(z_{i}\right)$ vertex operators is $((u-v) / 2)(\theta+\psi)$, so in order to reach the bra's weight of $((u-1) / 2)(\theta+\psi)$, the product of $\beta$ and $b c$ fields needs to contribute $((v-1) / 2)(\theta+\psi)$, that is, the only summands of $\prod_{i=1}^{v-1}\left(\beta\left(z_{i}\right) c\left(z_{i}\right)-b\left(z_{i}\right)\right)$ which contribute are those containing $\beta$ exactly $(v-1) / 2$ times. Further, since $\beta(z)=\mathrm{V}_{\theta+\psi}(z)$ and $\mathrm{V}_{-((u+v) / 2 v)(\theta+\psi)}(z)$ 
have regular operator product expansions with themselves and each other, the above matrix element simplifies to

$$
\begin{gathered}
\int \prod_{i \neq j}\left(1-\frac{z_{i}}{z_{j}}\right)^{\left(\xi^{2}-1\right) / 2} \mathrm{P}_{\kappa}^{2 /\left(\xi^{2}-1\right)}(z) \prod_{i=1}^{v-1} z_{i}^{2-(u+v) / 2} \\
\cdot \Delta(z)\left\langle\prod_{i=1}^{v-1}\left(c\left(z_{i}\right)-b\left(z_{i}\right)\right)\right\rangle_{\mathrm{NS}}^{+} \frac{\mathrm{d} z_{1} \cdots \mathrm{d} z_{v-1}}{z_{1} \cdots z_{v-1}} \\
=\int \prod_{i \neq j}\left(1-\frac{z_{i}}{z_{j}}\right)^{\left(\xi^{2}-1\right) / 2} \mathrm{P}_{\kappa}^{2 /\left(\xi^{2}-1\right)}(z) \mathrm{P}_{\kappa}^{-3}\left(z^{-1}\right) \frac{\mathrm{d} z_{1} \cdots \mathrm{d} z_{v-1}}{z_{1} \cdots z_{v-1}} \\
=\left\langle\mathrm{P}_{\kappa}^{2 /\left(\xi^{2}-1\right)}(z), \mathrm{P}_{\kappa}^{-3}(z)\right\rangle_{v-1}^{2 /\left(\xi^{2}-1\right)} \\
=\left\langle\mathrm{P}_{\kappa}^{2 /\left(\xi^{2}-1\right)}(z), \mathrm{P}_{\kappa}^{2 /\left(\xi^{2}-1\right)}(z)\right\rangle_{v-1}^{2 /\left(\xi^{2}-1\right)} \neq 0 .
\end{gathered}
$$

The case for $u$ and $v$ odd follows similarly by evaluating the matrix element

$$
\left\langle 0 ; \frac{u}{2}(\theta+\psi) ; \mathrm{NS}\left|b_{0} \rho_{\xi^{-1}}^{+}\left(\mathrm{P}_{\kappa}^{\frac{2}{\xi^{2}-1}}(y)\right) \mathcal{Q}_{2}^{[v-1]}\right|(1-v) \xi ;\left(u-\frac{u+v}{2 v}\right)(\theta+\psi) ; \mathrm{NS}\right\rangle
$$

and verifying that it is non-zero.

Lemma 28. Up to an irrelevant scale factor, the polynomial $g(h, \Sigma)$ of Theorem 9 is given by

$$
g(h, \Sigma)=\prod_{(i, j) \in \mathrm{K}_{u, v}^{\mathrm{NS}}}\left(\Sigma-s_{i, j}\right), \quad s_{i, j}=\frac{i}{2}-\frac{u j}{2 v} .
$$

In particular, $g(h, \Sigma)$ does not depend on $h$.

Proof. This proof mirrors that of Theorem 25 but with the screening operator $\mathcal{Q}_{1}$ replaced by $\mathcal{Q}_{2}$, that is, we will show that the factors $\left(\Sigma-s_{i, j}\right)$ all divide $g(h, \Sigma)$, thus saturating the degree bound on $g$ and determining $g$ up to scaling.

Recall that by Theorem 15 we have $\mathbb{F}_{[\lambda(\theta+\psi)-\psi]} \cong \mathcal{W}_{\lambda}$ as a $\beta \gamma$ module and so $|p ; j(\theta+\psi)-\psi ; \mathrm{NS}\rangle$ and $c_{0}|p ; j(\theta+\psi)-\psi ; \mathrm{NS}\rangle$ are $\widehat{\mathfrak{o s p}}(1 \mid 2)$ relaxed highest weight vectors. The zero mode of the singular vector shifts the $\widehat{\mathfrak{o s p}}(1 \mid 2)$ weights of such relaxed highest weight vectors by $(u-1) \alpha$. For $u$ and $v$ odd, this means a shift of $((u-1) / 2)(\theta+\psi)$ in $\beta \gamma$ weight, and for $u$ and $v$ even, it means a shift of $((u-2) / 2)(\theta+\psi)$ in $\beta \gamma$ weight and 1 unit of $b c$ weight.

Consider the case when $u$ and $v$ are odd, then the action of the zero mode of the singular vector on the relaxed highest weight vectors $|p ; j(\theta+\psi)-\psi ; \mathrm{NS}\rangle$ and $c_{0}|p ; j(\theta+\psi)-\psi ; \mathrm{NS}\rangle$ is non-zero if and only if the matrix elements

$$
\begin{aligned}
&\left\langle p ;\left(j+\frac{u-1}{2}\right)(\theta+\psi)-\psi ; \mathrm{NS}\right| \\
& \qquad \mathcal{Q}_{2}^{[v-1]} \mathrm{V}_{(1-v) \xi a+(u-(u+v) / 2 v)(\theta+\psi)}(w)|p ; j(\theta+\psi)-\psi ; \mathrm{NS}\rangle
\end{aligned}
$$


and

$$
\begin{aligned}
&\left\langle p ;\left(j+\frac{u-1}{2}\right)\right. \begin{array}{r}
(\theta+\psi)-\psi ; \mathrm{NS} \mid \\
b_{0} \mathcal{Q}_{2}^{[v-1]} \mathrm{V}_{(1-v) \xi a+(u-(u+v) / 2 v)(\theta+\psi)}(w) c_{0}
\end{array} \\
&|p ; j(\theta+\psi)-\psi ; \mathrm{NS}\rangle
\end{aligned}
$$

are non-zero. Evaluating the first matrix element gives

$$
\begin{aligned}
\left\langle p ;\left(j+\frac{u-1}{2}\right)(\theta+\psi)-\psi ; \mathrm{NS}\right| & \mathcal{Q}_{2}^{[v-1]} \mathrm{V}_{(1-v) \xi a+(u-(u+v) / 2 v)(\theta+\psi)}(w) \\
= & \prod_{i \neq j}\left(1-\frac{z_{i}}{z_{j}}\right)^{\left(\xi^{2}-1\right) / 2} \prod_{i=1}^{v-1}\left(1+\frac{z_{i}}{w}\right)^{\xi p} \prod_{i=1}^{v-1} z_{i}^{2-(u+v) / 2} \Delta(z) \\
& \cdot\left\langle\left(j+\frac{u-1}{2}\right)(\theta+\psi)-\psi ; \mathrm{NS}|| \begin{array}{l}
v-1 \\
\prod_{i=1}\left(\beta\left(z_{i}+w\right) c\left(z_{i}+w\right)-b\left(z_{i}+w\right)\right)
\end{array}\right. \\
\cdot \prod_{i=1}^{v-1} \mathrm{~V}_{-((u+v) / 2 v)(\theta+\psi)}\left(z_{i}+w\right) & \mid \mathrm{V}_{(u-(u+v) / 2 v)(\theta+\psi)}(w) \\
j(\theta+\psi)-\psi ; \mathrm{NS}\rangle & \frac{\mathrm{d} z_{1} \cdots \mathrm{d} z_{v-1}}{z_{1} \cdots z_{v-1}}
\end{aligned}
$$

The combined $\beta \gamma$ weight of the ket and the vertex operators in the integrand is $(j+(u-v) / 2)(\theta+\psi)-\psi$, so in order to reach the bra's weight of $(j+(u-1) / 2)$ $\cdot(\theta+\psi)$ the product of $\beta, b$ and $c$ fields needs to contribute $((v-1) / 2)(\theta+\psi)$, that is, after multiplying out the product, only summands containing $(v-1) / 2$ copies of $\beta$ will contribute to the matrix element. Thus, the matrix element inside the integrand evaluates to

$$
\begin{aligned}
& \left\langle\prod_{i=1}^{v-1}\left(c\left(z_{i}+w\right)\left(z_{i}+w\right)^{-1}-b\left(z_{i}+w\right)\right)\right\rangle_{\mathrm{NS}}^{+} w^{(u+v) / 2 v-u} \prod_{i=1}^{v-1}\left(z_{i}+w\right)^{(u+v) / 2 v} \\
& \quad=\left\langle\prod_{i=1}^{v-1}\left(c\left(z_{i}+w\right)\left(z_{i}+w\right)^{-1}-b\left(z_{i}+w\right)\right)\right\rangle_{\mathrm{NS}}^{+} w^{(v-u) / 2} \prod_{i=1}^{v-1}\left(1+\frac{z_{i}}{w}\right)^{(u+v) / 2 v}
\end{aligned}
$$

Thus the total matrix element evaluates to 


$$
\begin{aligned}
& \left\langle p ;\left(j+\frac{u-1}{2}\right)(\theta+\psi)-\psi ; \mathrm{NS}\right| \\
& \mathcal{Q}_{2}^{[v-1]} \mathrm{V}_{(1-v) \xi a+(u-(u+v) / 2 v)(\theta+\psi)}(w) \\
& |p ; j(\theta+\psi)-\psi ; \mathrm{NS}\rangle \\
& =w^{(v-u) / 2} \int \prod_{i \neq j}\left(1-\frac{z_{i}}{z_{j}}\right)^{\left(\xi^{2}-1\right) / 2} \prod_{i=1}^{v-1}\left(1+\frac{z_{i}}{w}\right)^{\xi p+(u+v) / 2 v} \prod_{i=1}^{v-1} z_{i}^{2-(u+v) / 2} \\
& \cdot \Delta(z)\left\langle\prod_{i=1}^{v-1}\left(c\left(z_{i}+w\right)\left(z_{i}+w\right)^{-1}-b\left(z_{i}+w\right)\right)\right\rangle_{\mathrm{NS}}^{+} \frac{\mathrm{d} z_{1} \cdots \mathrm{d} z_{v-1}}{z_{1} \cdots z_{v-1}} \\
& =(j)_{(v-1) / 2}(-1)^{(v-1) / 2} w^{(v-u) / 2} \int \prod_{i \neq j}\left(1-\frac{z_{i}}{z_{j}}\right)^{\left(\xi^{2}-1\right) / 2} \prod_{i=1}^{v-1}\left(1+\frac{z_{i}}{w}\right)^{\xi p+(u+v) / 2 v-1} \\
& \cdot \prod_{i=1}^{v-1} z_{i}^{-(u-v) / 2} \sum_{i=0}^{(v-1) / 2} w^{(v-1) / 2-i} c_{i}^{(v-1)} \mathrm{P}_{\left[v-2-\epsilon^{(v-1)}(i)\right]}^{-3}\left(z^{-1}\right) \frac{\mathrm{d} z_{1} \cdots \mathrm{d} z_{v-1}}{z_{1} \cdots z_{v-1}} \\
& =\sum_{i=1}^{(v-1) / 2} w^{(v-1) / 2-i} c_{i}^{(v-1)} \\
& \cdot\left\langle\mathrm{P}_{\left[(u+v) / 2+2-\epsilon^{(v-1)}(i)\right]}^{-3}(z), \prod_{j=1}^{v-1}\left(1+\frac{z_{j}}{w}\right)^{\xi p+(u+v) / 2 v-1}\right\rangle_{v-1}^{2 /\left(\xi^{2}-1\right)}
\end{aligned}
$$

where the second equality makes use of the identities of (32). As in the proof of Theorem 25, we can look for zeros common to all summands to find zeros of the action of the zero mode of the singular vector by identifying cells common to all Young diagrams of partitions dominated by the $\left[(u+v) / 2+2-\epsilon^{(v-1)}(i)\right]$. By [34, Lem. 3.2.(3)] these cells form the length $v-1$ partition

$$
\rho=\left[\frac{u-1}{2}, \frac{u-1}{2}-1, \frac{u-1}{2}-1, \ldots, \frac{u-v}{2}+1, \frac{u-v}{2}+1, \frac{u-v}{2}\right]
$$

and the zeros common to all summands above include those of

$$
\begin{aligned}
\prod_{b \in \rho}\left(\xi p+\frac{\xi^{2}-1}{2}-a^{\prime}(b)+\frac{\xi^{2}-1}{2} \ell^{\prime}(b)\right) & =\prod_{b \in \rho}\left(s_{p}-s_{\ell^{\prime}(b)+2 a^{\prime}(b)+2, \ell^{\prime}(b)+1}\right) \\
& =\prod_{\substack{(i, j) \in \mathrm{K}_{u, v}^{\mathrm{NS}} \\
i>j}}\left(s_{p}-s_{i, j}\right) .
\end{aligned}
$$

The analogous matrix element coming from the action of the zero mode of the singular vectors in the relaxed highest weight vector $c_{0}|p ; j(\theta+\psi)-\psi ; \mathrm{NS}\rangle$ gives 


$$
\begin{gathered}
\left\langle p ;\left(j+\frac{u-1}{2}\right)(\theta+\psi)-\psi ; \mathrm{NS}\right| \\
b_{0} \mathcal{Q}_{2}^{[v-1]} \mathrm{V}_{(1-v) \xi a+(u-(u+v) / 2 v)(\theta+\psi)}(w) c_{0} \\
=w^{(v-u) / 2}\left\langle\prod_{i \neq j}\left(1-\frac{z_{i}}{z_{j}}\right)^{\left(\xi^{2}-1\right) / 2} \prod_{i=1}^{v-1}\left(1+\frac{z_{i}}{w}\right)^{\xi p+(u+v) / 2 v} \prod_{i=1}^{v-1} z_{i}^{2-(u+v) / 2}\right. \\
\quad \sum_{i=1}^{(v-1) / 2} w^{(v-1) / 2-i c_{i}^{(v-1)}}\left\langle\prod_{i=1}^{v-1}\left(c\left(z_{i}+w\right)\left(z_{i}+w\right)^{-1}-b\left(z_{i}+w\right)\right)\right\rangle_{\mathrm{NS}} \frac{\mathrm{d} z_{1} \cdots \mathrm{d} z_{v-1}}{z_{1} \cdots z_{v-1}} \\
\cdot\left\langle\mathrm{P}_{\left[(u+v) / 2+2-\epsilon^{(v-1)}(i)\right]}^{-3}(z), \prod_{j=1}^{v-1}\left(1+\frac{z_{j}}{w}\right)^{\xi p+(u+v) / 2 v-1}\right\rangle_{v-1}^{2 /\left(\xi^{2}-1\right)}
\end{gathered}
$$

As above, the zeros common to all summands include those of

$$
\begin{aligned}
\prod_{b \in \rho}\left(\xi p+\frac{\xi^{2}-1}{2}-a^{\prime}(b)+\frac{\xi^{2}-1}{2} \ell^{\prime}(b)\right) & =\prod_{b \in \rho}\left(-s_{p}-s_{\ell^{\prime}(b)+2 a^{\prime}(b)+2, \ell^{\prime}(b)+1}\right) \\
& =\prod_{\substack{(i, j) \in \mathrm{K}_{u, v}^{\mathrm{NS}} \\
i>j}}\left(-s_{p}-s_{i, j}\right) .
\end{aligned}
$$

Thus, $g\left(h, s_{i, j}\right)=0$ independent of $h$ for all $(i, j) \in \mathrm{K}_{u, v}^{\mathrm{NS}}$. Note that we have used the fact that $u>v$, otherwise the ordered pairs $(i, j)$ and $(u-i, v-j)$, with $i>j$, would not cover the entire Neveu-Schwarz Kac table.

For $u$ and $v$ even, the action of the zero mode of the singular vector on the relaxed highest weight vectors $|p ; j(\theta+\psi)-\psi ; \mathrm{NS}\rangle$ and $c_{0}|p ; j(\theta+\psi)-\psi ; \mathrm{NS}\rangle$ is non-zero if and only if the matrix elements

$$
\begin{gathered}
\left\langle p ;\left(j+\frac{u-2}{2}\right)(\theta+\psi)-\psi ; \mathrm{NS}\left|b_{0} \mathcal{Q}_{2}^{[v-1]} \mathrm{V}_{(1-v) \xi a+(u-(u+v) / 2 v)(\theta+\psi)}(w)\right| p ; j(\theta+\psi)-\psi ; \mathrm{NS}\right\rangle \\
\left\langle p ;\left(j+\frac{u}{2}\right)(\theta+\psi)-\psi ; \mathrm{NS}\left|\mathcal{Q}_{2}^{[v-1]} \mathrm{V}_{(1-v) \xi a+(u-(u+v) / 2 v)(\theta+\psi)}(w) c_{0}\right| p ; j(\theta+\psi)-\psi ; \mathrm{NS}\right\rangle
\end{gathered}
$$

are non-zero. By the same reasoning as in the case of odd $u$ and $v$ above, these matrix elements both evaluate to

$$
\sum_{i=0}^{v / 2-1} w^{-u / 2-i} c_{i}\left\langle\prod_{i=1}^{v-1} z_{i}^{(u-v) / 2} \mathrm{P}_{\left[v-2-\epsilon^{(v-1)}(i)\right]}^{-3}(z), \prod_{i=1}^{v-1}\left(1+\frac{z_{i}}{w}\right)^{\xi p+\left(\xi^{2}+1\right) / 2-1}\right\rangle_{v-1}^{2 /\left(\xi^{2}-1\right)} .
$$


By [34, Lem. 3.2.(4)], the cells common to all Young diagrams dominated by $\left[v-2-\epsilon^{(v-1)}(i)\right]$ form the length $v-1$ partition

$$
\rho=\left[\frac{u}{2}-1, \frac{u}{2}-1, \ldots, \frac{u-v}{2}+1, \frac{u-v}{2}+1, \frac{u-v}{2}\right] .
$$

The zeros common to all summands therefore include those of

$$
\prod_{b \in \rho}\left( \pm s_{p}-s_{\ell^{\prime}(b)+2 a^{\prime}(b)+2, \ell^{\prime}(b)+1}\right) .
$$

Thus, $g\left(h, s_{i, j}\right)=0$ independent of $h$ for all $(i, j) \in \mathrm{K}_{u, v}^{\mathrm{NS}}$.

Lemma 29. Up to an irrelevant scale factor, the polynomial $g_{\tau}(h, Q)$ of Theorem 9 is given by

$$
g_{\tau}(h, Q)=\prod_{[(i, j)] \in \overline{\mathrm{K}}_{u, v}^{\mathrm{R}}}\left(Q-q_{i, j}\right), \quad q_{i, j}=\frac{(u j-v i)^{2}-4 v^{2}}{8 v^{2}} .
$$

In particular, $g_{\tau}(h, Q)$ does not depend on $h$.

Proof. This proof mirrors that of Theorem 26 but with the screening operator $\mathcal{Q}_{1}$ replaced by $\mathcal{Q}_{2}$. For $u$ and $v$ odd, the action of the zero mode of the singular vector on the candidate relaxed highest weight vector is non-zero if and only if the following matrix element is non-zero:

$$
\begin{aligned}
& \left\langle p ;\left(j+\frac{u-1}{2}\right)(\theta+\psi)-\psi ; \mathrm{R}\right| \\
& \mathcal{Q}_{2}^{[v-1]} \mathrm{V}_{(1-v) \xi a+(u-(u+v) / 2 v)(\theta+\psi)}(w) \\
& =w^{(1-u) / 2}(-2)^{(v-1) / 2} / \prod_{i \neq j}\left(1-\frac{z_{i}}{z_{j}}\right)^{\left(\xi^{2}-1\right) / 2} \prod_{i=1}^{v-1}\left(1+\frac{z_{i}}{w}\right)^{\xi p} \prod_{i=1}^{v-1} z_{i}^{2-(u+v) / 2} \Delta(z) \\
& \cdot\left\langle\left(j+\frac{u-1}{2}\right)(\theta+\psi)-\psi ; \mathrm{R}\right| \prod_{i=1}^{v-1}\left(\beta\left(z_{i}+w\right) c\left(z_{i}+w\right)-b\left(z_{i}+w\right)\right) \\
& \cdot \prod_{i=1}^{v-1} \mathrm{~V}_{-((u+v) / 2 v)(\theta+\psi)}\left(z_{i}+w\right) \cdot \mathrm{V}_{(u-(u+v) / 2 v)(\theta+\psi)}(w) \\
& =w^{(1-u) / 2}(-2)^{(v-1) / 2}|j(\theta+\psi)-\psi ; \mathrm{R}\rangle \frac{\mathrm{d} z_{1} \cdots \cdot \mathrm{d} z_{v-1}}{z_{1} \cdots z_{v-1}} \\
& \cdot\left\langle\mathrm{P}_{\delta^{(v-1)}((u-v) / 2+1,(u-v) / 2+1)}(z), \prod_{i=1}^{v-1}\left(1+\frac{z_{i}}{w}\right)^{\xi p+\xi^{2} / 2}\right\rangle_{v-1}^{2 /\left(\xi^{2}-1\right)}
\end{aligned}
$$

where the matrix element in the integrand is evaluated as in the proof of Theorem 28 (that is, the only summands of the product of $\beta, b$ and $c$ fields which 
contribute are those containing $(v-1) / 2$ copies of $\beta)$ and using the identities of (33). As above in the proofs of Theorem 25,26 , and 28 , we identify cells common to all Young diagrams of partitions dominated by $\delta^{(v-1)}((u-v) / 2+1,(u-v) / 2+1)$ to find zeros. By [34, Lem.3.2.(1)] the cells form the length $v-1$ partition

$$
\rho=\left[\frac{u-1}{2}, \frac{u-1}{2}, \frac{u-1}{2}-1, \frac{u-1}{2}-1, \ldots, \frac{u-v}{2}+1, \frac{u-v}{2}+1\right] .
$$

The zeros of the matrix element (50) therefore include those of

$$
\begin{aligned}
\prod_{b \in \rho}\left(\xi p+\frac{\xi^{2}}{2}\right. & \left.-a^{\prime}(b)+\frac{\xi^{2}-1}{2} \ell^{\prime}(b)\right) \\
& =\prod_{b \in \rho}\left(\xi p+\frac{1}{2}-\frac{1}{2}\left(\ell^{\prime}(b)+2 a^{\prime}(b)+1\right)+\frac{\xi^{2}}{2}\left(\ell^{\prime}(b)+1\right)\right) \\
& =\prod_{b \in \rho}\left(s_{p}-s_{\left.\ell^{\prime}(b)+2 a^{\prime}(b)+1, \ell^{\prime}(b)+1\right)}=\prod_{\substack{(i, j) \in \mathrm{K}_{u, v}^{\mathrm{R}} \\
i>j}}\left(s_{p}-s_{i, j}\right),\right.
\end{aligned}
$$

Also as in the proof of Theorem 26, $\left(s_{p}-s_{i, j}\right)$ dividing $g_{\tau}\left(h, q_{p}\right)$ implies that $\left(s_{p}+s_{i, j}\right)=\left(s_{p}-s_{u-r, v-s}\right)$ does so as well and thus $q_{p}-q_{i, j}$ divides $g_{\tau}\left(h, q_{p}\right)$ for all $(i, j) \in \mathrm{K}_{u, v}^{\mathrm{R}}, i>j$. Since $u>v$ every class of $\overline{\mathrm{K}}_{u, v}^{\mathrm{R}}$ has a representative of this form, thus proving the lemma for odd $u$ and $v$.

For $u$ and $v$ even, one must consider the action of the zero mode of the $y_{0}$ descendant of the singular vector. This action vanishes on the relaxed highest weight vector $|p ; j(\theta+\psi)-\psi ; \mathbf{R}\rangle$ if and only if the matrix element

$$
\begin{aligned}
\left\langle p ;\left(j+\frac{u-2}{2}\right)(\theta+\psi)-\psi ; \mathrm{R}\right| & \\
\mathcal{Q}_{2}^{[v-1]} c(w) \mathrm{V}_{(1-v) \xi a+(u-(u+v) / 2 v)(\theta+\psi)}(w) & |p ; j(\theta+\psi)-\psi ; \mathrm{R}\rangle
\end{aligned}
$$

vanishes. This matrix element evaluates to

$$
\begin{aligned}
& \left\langle p ;\left(j+\frac{u-2}{2}\right)(\theta+\psi)-\psi ; \mathrm{R}\right| \\
& \mathcal{Q}_{2}^{[v-1]} c(w) \mathrm{V}_{(1-v) \xi a+(u-(u+v) / 2 v)(\theta+\psi)}(w) \\
& =-w^{1-u / 2}(-2)^{(v-2) / 2} \\
& |p ; j(\theta+\psi)-\psi ; \mathrm{R}\rangle \\
& \cdot\left\langle\mathrm{P}_{\delta^{(v-1)}((u-v) / 2+1,(u-v) / 2+1)}^{-3}(z), \prod_{i=1}^{v-1}\left(1+\frac{z_{i}}{w}\right)^{\xi p+\xi^{2} / 2}\right\rangle_{v-1}^{2 /\left(\xi^{2}-1\right)}
\end{aligned}
$$

The usual trick of looking for cells common to all Young diagrams of partitions dominated by $\delta^{(v-1)}((u-v) / 2+1,(u-v) / 2+1)$ gives the length $v-1$ partition

$$
\rho=\left[\frac{u}{2}, \frac{u}{2}-1, \frac{u}{2}-1, \frac{u}{2}-2, \frac{u}{2}-2, \ldots, \frac{u-v}{2}+1, \frac{u-v}{2}+1\right]
$$


by $[34$, Lem. 3.2.(2)]. The zeros of the above matrix element therefore include those of

$$
\prod_{b \in \rho}\left(s_{p}-s_{\ell^{\prime}(b)+2 a^{\prime}(b)+1, \ell^{\prime}(b)+1}\right)=\prod_{\substack{(i, j) \in \mathrm{K}_{u, v}^{\mathrm{R}} \\ i>j}}\left(s_{p}-s_{i, j}\right) .
$$

As in the case of odd $u$ and $v$ this implies that $g_{\tau}\left(h, q_{i, j}\right)=0$ for all $[(i, j)] \in \overline{\mathrm{K}}_{u, v}^{\mathrm{R}}$.

\section{References}

[1] V. Kac, Lie superalgebras, Adv. Math. 26 (1977), no. 1, 8-96.

[2] D. Ridout, J. Snadden, S. Wood, An admissible level $\widehat{\mathfrak{o s p}}(1 \mid 2)$-model: modular transformations and the Verlinde formula, Lett. Math. Phys. 108 (2018), no. 11, 23632423.

[3] J.-B. Fan, M. Yu, Modules over affine Lie superalgebras, arXiv:hep-th/9304122 (1993).

[4] I. P. Ennes, A. V. Ramallo, Fusion rules and singular vectors of the osp $(1 \mid 2)$ current algebra, Nucl. Phys. B502 (1997), no. 3, 671-712.

[5] V. Kac, W. Wang, Vertex operator superalgebras and their representations, in: Mathematical Aspects of Conformal and Topological Field Theories and Quantum Groups, Contemporary Mathematics, Vol. 175, American Mathematical Society, Providence, 1994, pp. 161-191.

[6] T. Creutzig, J. Frohlich, S. Kanade, Representation theory of $L_{k}(\mathfrak{o s p}(1 \mid 2))$ from vertex tensor categories and Jacobi forms, Proc. Amer. Math. Soc. 146 (2018), no. 11, 4571-4589.

[7] V. Kac, M. Wakimoto, Modular invariant representations of infinite-dimensional Lie algebras and superalgebras, Proc. Nat. Acad. Sci. USA 85 (1988), no. 14, 4956-4960.

[8] K. Kawasetsu, D. Ridout, Relaxed highest-weight modules I: rank 1 cases, Comm. Math. Phys. 368 (2019), no. 2, 627-663.

[9] D. Ridout, $\widehat{\mathfrak{s l}}(2)_{-1 / 2}$ : A case study, Nucl. Phys. B814 (2009), no. 3, 485-521.

[10] T. Creutzig, D. Ridout, Modular data and Verlinde formulae for fractional level WZW models I, Nucl. Phys. B865 (2012), no. 1, 83-114.

[11] T. Creutzig, D. Ridout, Modular data and verlinde formulae for fractional level WZW models II, Nucl. Phys. B875 (2013), no. 2, 423-458.

[12] S. Kanade, T. Liu, D. Ridout, Cosets, characters and fusion for the admissible level $\mathfrak{o s p}(1 \mid 2)$ minimal models, Nucl. Phys. B938 (2019), no. , 22-55.

[13] D. Adamović, Realizations of simple affine vertex algebras and their modules: the cases sl(2) and osp(1,2), Comm. Math. Phys. 366 (2019), no. 3, 1025-1067.

[14] B. Feigin, T. Nakanishi, H. Ooguri, The annihilating ideals of minimal models, Int. J. Mod. Phys. A7 (1992), no. 1, 217-238.

[15] Y. Zhu, Modular invariance of characters of vertex operator algebras, J. Amer. Math. Soc. 9 (1996), no. 1, 237-302.

[16] K. Iohara, Y. Koga, Enright functors for Kac-Moody superalgebra, Abh. Math. Semin. Univ. Hambg. 82 (2012), no. 2, 205-226. 
[17] Ф. Г. Маликов, Б. Л. Фейгин, Д. Б. Фукс, Особые векторы в модулях Верма над алгебрами Кача-Муди, Функц. анализ и его прил. 20 (1986), вып. 2, 25-37. Engl. transl.: F. G. Malikov, B. L. Feigin, D. B. Fuks, Singular vectors in Verma modules over Kac-Moody algebras, Funct. Anal. Appl. 20 (1986), no. 2, 103-113.

[18] B. Feigin, D. Fuchs, Representations of the Virasoro algebra, in: Representation of Lie groups and Related Topics, Advanced Studies in Contemporary Mathematics, Vol. 7, Gordon and Breach, New York, 1990, pp. 465-554.

[19] M. Wakimoto, Fock representations of the affine Lie algebra $A_{1}^{(1)}$, Comm. Math. Phys. 104 (1986), no. 4, 605-609.

[20] V. Dotsenko, V. Fateev, Conformal algebra and multipoint correlation functions in $2 D$ statistical models, Nucl. Phys. B240 (1984), no. 3, 312-348.

[21] A. Tsuchiya, Y. Kanie, Fock space representations of the Virasoro algebra-intertwining operators, Publ. Res. Inst. Math. Sci. 22 (1986), no. 2, 259-327.

[22] M. Wakimoto, Y. Yamada, The Fock representations of the Virasoro algebra and the Hirota equations of the modified KP hierarchies, Hiroshima Math. J. 16 (1986), no. 2, 427-441.

[23] K. Mimachi, Y. Yamada, Singular vectors of the Virasoro algebra in terms of Jack symmetric polynomials, Comm. Math. Phys. 174 (1995), no. 2, 447-455.

[24] M. Kato,Y. Yamada, Missing link between Virasoro and $\widehat{s l(2)}$ Kac-Moody algebras, Progr. Theoret. Phys. Suppl. 110 (1992), no. 110, 291-302.

[25] D. Ridout, S. Wood, Relaxed singular vectors, Jack symmetric functions and fractional level $\widehat{\mathfrak{s l}}(2)$ models, Nucl. Phys. B894 (2015), 621-664.

[26] P. Desrosiers, L. Lapointe, P. Mathieu, Supersymmetric Calogero-Moser-Sutherland models and Jack superpolynomials, Nucl. Phys. B606 (2001), no. 3, 547-582.

[27] S. Yanagida, Singular vectors of $N=1$ super Virasoro algebra via Uglov symmetric functions, arXiv:1508.06036 (2015).

[28] O. Blondeau-Fournier, P. Mathieu, D. Ridout, S. Wood, The super-Virasoro singular vectors and Jack superpolynomials relationship revisited, Nucl. Phys. B913 (2016), $34-63$.

[29] H. Awata, Y. Matsuo, S. Odake, J. Shiraishi, Excited states of the Calogero-Sutherland model and singular vectors of the $W_{n}$ algebra, Nucl. Phys. B449 (1995), no. $1-2,347-374$.

[30] D. Ridout, S. Siu, S. Wood, Singular vectors for the $W_{N}$ algebras, J. Math. Phys., 59 (2018), no. 3, 031701.

[31] B. Feigin, M. Jimbo, T. Miwa, E. Mukhin, A differential ideal of symmetric polynomials spanned by Jack polynomials at $\beta=-(r-1) /(k+1)$, Int. Math. Res. Not. 2002 (2002), no. 23, 1223-1237.

[32] A. Tsuchiya, S. Wood, On the extended $W$-algebra of type $\mathfrak{s l}_{2}$ at positive rational level, Int. Math. Res. Not. 2015 (2015), no. 14, 5357-5435.

[33] D. Ridout, S. Wood, From Jack polynomials to minimal model spectra, J. Phys. A48 (2015), no. 4, 045201.

[34] O. Blondeau-Fournier, P. Mathieu, D. Ridout, S. Wood, Superconformal minimal models and admissible Jack polynomials, Adv. Math. 314 (2017), 71-123.

[35] S.-J. Chen, W. Wang, Dualities and Representations of Lie Superalgebras, Graduate Studies in Mathematics, Vol. 144, American Mathematical Society, Providence, 2012. 
[36] G. Pinczon, The enveloping algebra of the lie superalgebra osp(1|2), J. Algebra 132 (1990), no. 1, 219-242.

[37] A. Leśniewski, A remark on the Casimir elements of Lie superalgebras and quantized Lie superalgebras, J. Math. Phys. 36 (1995), no. 3, 1457-1461.

[38] R. Block, Classification of the irreducible representations of $\mathfrak{s t}(2, \mathbb{C})$, Bull. Amer. Math. Soc. 1 (1972), no. 1, 247-250.

[39] R. Block, The irreducible representations of the Weyl algebra $A_{1}$, Lecture Notes in Mathematics $\mathbf{7 4 0}$ (1979) , 69-79.

[40] V. Mazorchuk, Lectures on $\mathfrak{s l}_{2}(\mathbb{C})$-Modules, Imperial College Press, London, 2010.

[41] D. Ridout, S. Wood, Bosonic ghosts at $c=2$ as a logarithmic CFT, Lett. Math. Phys. 105 (2015), no. 2, 279-307.

[42] E. Frenkel, D. Ben-Zvi, Vertex Algebras and Algebraic Curves, Mathematical Surveys and Monographs, Vol. 88, American Mathematical Society, Providence, 2001.

[43] M. Gorelik, V. Kac, On simplicity of vacuum modules, Adv. Math. 211 (2007), no. $2,621-677$.

[44] C. Dong, H. Li, G. Mason, Twisted representations of vertex operator algebras, Math. Ann. 310 (1998), no. 3, 571-600.

[45] I. Frenkel, Y. Zhu, Vertex operator algebras associated to representations of affine and Virasoro algebras, Duke. Math. J. 66 (1992), no. 1, 123-168.

[46] K. Iohara, Y. Koga, Fusion algebras for $N=1$ superconformal field theories through coinvariants I: $\widehat{\mathfrak{o s p}}(1 \mid 2)$-symmetry, J. reine angew. Math. 531 (2001), 1-34.

[47] V. Kac, Vertex Algebras for Beginners, 2nd edition, University Lecture Series, Vol. 10, American Mathematical Society, Providence, 1998.

[48] C. Dong, J. Lepowsky, Generalized Vertex Algebras and Relative Vertex Operators, Progress in Mathematics, Vol. 112, Birkhäuser, Boston, 1993.

[49] M. Bershadsky, H. Ooguri, Hidden $\operatorname{OSp}(N, 2)$ symmetries in superconformal field theories, Phys. Lett. B229 (1989), no. 4, 374-378.

[50] M. Bershadsky, H. Ooguri, Hidden SL $(n)$ symmetry in conformal field theories, Comm. Math. Phys. 126 (1989), no. 1, 49-83.

[51] J. L. Petersen, J. Rasmussen, M. Yu, Conformal blocks for admissible representations in SL(2) current algebra, Nucl. Phys. B458 (1995), no. 1-2, 309-342.

[52] I. Macdonald, Symmetric Functions and Hall Polynomials, 2nd edition, Oxford Mathematical Monographs, Clarendon Press, Oxford, 1995.

Open Access This article is licensed under a Creative Commons Attribution 4.0 International License, which permits use, sharing, adaptation, distribution and reproduction in any medium or format, as long as you give appropriate credit to the original author(s) and the source, provide a link to the Creative Commons licence, and indicate if changes were made. The images or other third party material in this article are included in the article's Creative Commons licence, unless indicated otherwise in a credit line to the material. If material is not included in the article's Creative Commons licence and your intended use is not permitted by statutory regulation or exceeds the permitted use, you will need to obtain permission directly from the copyright holder. To view a copy of this licence, visit http://creativecommons.org/ licenses/by/4.0/.

Publishers Note Springer Nature remains neutral with regard to jurisdictional claims in published maps and institutional affiliations. 